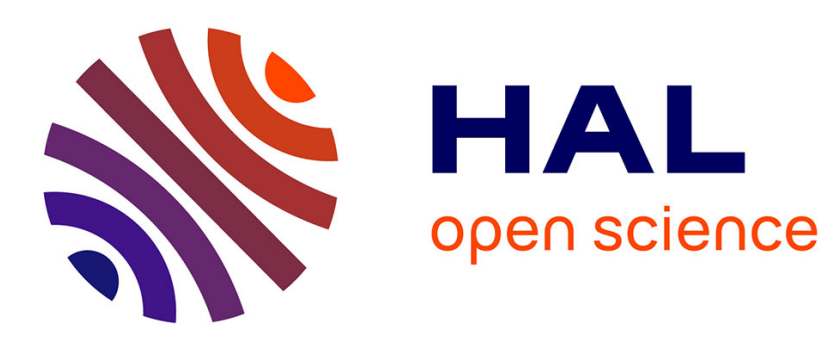

\title{
Albert Gaudry et les vertébrés fossiles du Luberon. Histoire d'une collection de référence
}

Vincent Pernègre, Pascal Tassy

\section{To cite this version:}

Vincent Pernègre, Pascal Tassy. Albert Gaudry et les vertébrés fossiles du Luberon. Histoire d'une collection de référence. Geodiversitas, 2014, 36 (4), pp.623-667. 10.5252/g2014n4a5 . hal-01324872

\section{HAL Id: hal-01324872 https://hal.sorbonne-universite.fr/hal-01324872}

Submitted on 1 Jun 2016

HAL is a multi-disciplinary open access archive for the deposit and dissemination of scientific research documents, whether they are published or not. The documents may come from teaching and research institutions in France or abroad, or from public or private research centers.
L'archive ouverte pluridisciplinaire HAL, est destinée au dépôt et à la diffusion de documents scientifiques de niveau recherche, publiés ou non, émanant des établissements d'enseignement et de recherche français ou étrangers, des laboratoires publics ou privés. 


\title{
Albert Gaudry et les vertébrés fossiles du Luberon. Histoire d'une collection de référence
}

\author{
Vincent PERNÈGRE \\ Muséum national d'Histoire naturelle, \\ Direction des Collections, UGC de Paléontologie, \\ case postale 38, 57 rue Cuvier, F-75231 Paris cedex 05 (France) \\ pernegre@mnhn.fr \\ Pascal TASSY \\ Muséum national d'Histoire naturelle, \\ Département Histoire de la Terre, USM203/UMR7207 CNRS MNHN UPMC \\ Centre de Recherches sur la Paléobiodiversité \\ et les Paléoenvironnements (CR2P) \\ case postale 38, 57 rue Cuvier, F-75231 Paris cedex 05 (France) \\ ptassy@mnhn.fr
}

\author{
MOTS CLÉS \\ Albert Gaudry, \\ Mammalia, \\ Miocène, \\ Luberon, \\ collection, \\ conservation, \\ paléontologie.
}

Pernègre V. \& Tassy P. 2014. - Albert Gaudry et les vertébrés fossiles du Luberon. Histoire d'une collection de référence. Geodiversitas 36 (4): 623-667. http://dx.doi.org/10.5252/g2014n4a5

\section{RÉSUMÉ}

La collection des vertébrés fossiles du Luberon et la publication qui en a été tirée par Albert Gaudry sont fondatrices de la paléontologie évolutionniste et, plus généralement de la paléontologie moderne. On examine les modalités de la gestion de cette collection depuis près de 150 ans, notamment en ce qui concerne les mammiferes qui forment le gros de la collection.

\section{EXTENDED ABSTRACT}

Albert Gaudry and the fossil vertebrates from Luberon. The history of a reference collection. The collection of fossil vertebrates from Luberon on the one hand, and the description of the fossil animals from "Mont Léberon" by Gaudry, Fischer \& Tournouër in 1873 on the other, played a major role in the emergence in France of what can be called evolutionary palaeontology. Gaudry decided to excavate the locality of Luberon (also called Cucuron) to compare its fauna to that of Pikermi described by him a few years before. In the section of the monograph devoted to vertebrates, Gaudry especially developed the biostratigraphical, paleobiogeographical, and evolutionary aspects of the fauna in a very modern manner. From the evolutionary viewpoint, Gaudry discussed the question of identifying chronological and/or geographical "races", that is subspecies, and species. This genuine work is mainly based on the collection housed in the Muséum 
KEY WORDS

Albert Gaudry,

Mammalia,

Miocene,

Luberon,

Collection,

Curation,

Palaeontology. national d'Histoire naturelle, Paris (MNHN), so that both the monograph and the collection are milestones in the history of palaeontology. The fate of the collection since Gaudry's time is analyzed from the curators' viewpoint. The previous state of the fossils is described as well their restoration.

The fossils were previously disseminated in several areas of the palaeontology building due to their different sizes. They are now located in one place opened for study and revision (a few is exhibited in the public gallery). The basic cleaning and restoration of the material was also associated with the taxonomic and geographic identification since several specimens studied by Gaudry had been in the past erroneously transferred to other localities such as Maragheh (Iran). One explanation, except previous poor curation, is the greyish colour due to dust, decades after decades, that makes the different late Miocene fossils of various localities look alike (and even from middle Miocene). One example is one exquisite cranium of Gazella deperdita (now MNHN.F.LUB681) figured by Gaudry in his monograph dated 1873, which bore other undue numbers with the MAR acronym for Maragheh (Maragha in the French litterature), and previously thought to be lost.

Once cleaned, the specimens were restored and consolidated with an acrylic emulsion (a methyl methacrylate/n-butyl methacrylate polymer), with different concentrations depending on different operations. Generally speaking the collection was in a very bad state and numerous specimens figured by Gaudry broken and incomplete since their description. The worst case is a specimen broken in seven parts. As an example, let us cite one tibia-fibula of the rhinocerotid Dihoplus schleiermacheri figured by Gaudry was found in six pieces, some of them discovered in a drawer containing miscellaneous middle and late Miocene mammal fragments. Others were not even completely separated from the matrix, a situation not always demonstrated by Gaudry's drawings which are sometimes more reconstructions then mere reproduction. Ultraviolet light was used to identify old hand writing. Many fossils of Gaudry's collection from Luberon bore the number 156, but not all. This number corresponds to the first catalogue number of Gaudry's collection. Other specimens from Luberon are from Christol, Caire, Conil, Lartet, Saporta and Depéret's collections (some Saporta's specimen bore also Gaudry's number 156). All the collection is now labelled with the acronym LUB for Luberon, that is, 988 numbers for 1201 elements. The electronic catalogue is complete and available online at the address: http://science.mnhn.fr/institution/mnhn/collection/f/item/search/form/ For the first time since 1873 , the entire set housed in the collection of palaeontology is available. Although a rather modest collection, its expertise, restoration, and electronic catalogue were time consuming. Circa 700 hours were spent during the last two years on the curation of the material from Luberon. This timing gives an idea of what is needed for the whole collection of fossil mammals (between 300000 and 500000 specimens). Yet, as it is, the Luberon collection is ready for the next century, and after (as the other fossil mammal Late Miocene localities). More generally speaking, restorations of specimens such as these from Luberon, are the basis of curation in palaeontology and, ironically, are in contradiction with present strategies of collection managing which favour data banks somehow disconnected from the specimens themselves. 


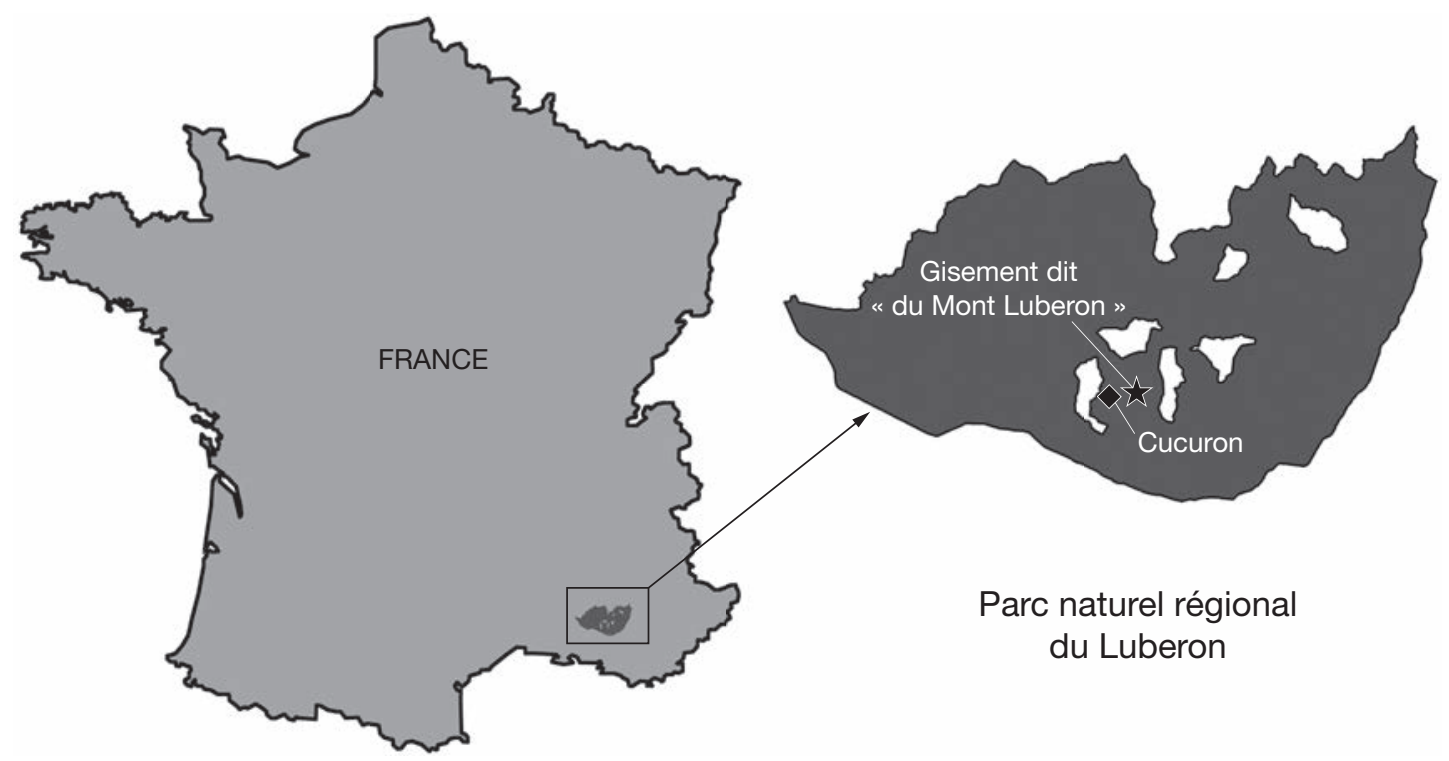

FIG. 1. - Localisation du gisement dit « du Luberon » ou « du Mont Luberon », ou encore « de Cucuron », dans le parc naturel régional du Luberon.

\section{INTRODUCTION}

En 1873 Albert Gaudry publie avec Paul Fischer et Raoul Tournouër l'étude des Animaux fossiles $d u$ Mont Léberon (Vaucluse), une faune d'âge Miocène (Gaudry et al. 1873). Son propre travail (Gaudry 1873) est consacré aux vertébrés et notamment aux mammiferes, dans la suite de son volume sur la faune de Pikermi dont la publication s'est achevée six ans plus tôt. Gaudry (1873: 5) présente ainsi son travail : "J'ai cru qu'en poursuivant l'étude des espèces de Pikermi dans une région éloignée de cette localité, je pourrais jeter quelques lumières sur la question des races fossiles. Ce motif m'a engagé à faire des fouilles dans le mont Léberon et à présenter aux naturalistes le mémoire qui va suivre ".

Gaudry est connu pour être le premier paléontologue évolutionniste en France, sinon darwinien. À propos de sa théorie de la descendance avec modification, dans une lettre à Quatrefages, Darwin lui-même cite Gaudry comme le seul zoologiste Français qui le défende : " [...] in France I have not heard of a single zoologist, except M. Gaudry (and he only partially), who supports my views "
(Darwin 1888: 118). Gaudry est un paléontologue complet : homme de terrain, auteur de traités (Gaudry 1878, 1883, 1890), muséologue (il est le concepteur de l'actuelle galerie de paléontologie du Muséum), il est aussi le premier à avoir publié des arbres phylogénétiques associant espèces éteintes et actuelles (Gaudry 1866), appliquant le modèle darwinien de filiation (Tassy 2006, 2011).

Après ses fouilles paléontologiques à Pikermi en Grèce, Gaudry se rend dans le Sud de la France afin de conduire le même travail de terrain, cette fois dans les sédiments tertiaires du mont Luberon dans le Vaucluse (mont Léberon, comme on disait alors, voire Liberon selon Christol (1832: 181); l'orthographe "Lubéron " étant fautive, quoiqu'admise par le dictionnaire et la plus fréquente aujourd'hui). Le gisement se situe à $4 \mathrm{~km}$ du village de Cucuron (c'est pourquoi la localité est aussi souvent appelée Cucuron). Aujourd'hui le site se trouve dans le parc naturel régional du Luberon, créé en 1977, qui s'étend sur deux départements, Vaucluse et Alpes de Haute Provence (Fig. 1). On peut remarquer qu'une figure publiée par Gaudry orne discrètement le sujet " recherche scientifique " du site internet 
du parc : http://reserve-naturelle.parcduluberon.fr/ Activites-scientifiques/Recherche-scientifique/

La monographie que Gaudry tire de son travail est un ouvrage fondateur. À partir de sa conception phylogénétique de la paléontologie, déjà appliquée dans l'étude des mammifères de Pikermi (Gaudry 1862-1867), le paléontologue français étend cette fois ses analyses à une problématique liée à la stratigraphie en milieu continental, à des comparaisons biostratigraphiques et paléoenvironnementales (Gaudry 1873).

De la sorte, la collection constituée par Gaudry et conservée au Muséum national d'Histoire naturelle est devenue une référence dans l'histoire de la paléontologie moderne. Une référence toutefois passée relativement inaperçue en dehors des spécialistes des faunes du Miocène supérieur. Or la conception de ce travail dépasse largement son seul sujet.

Dans le présent article nous nous proposons en premier d'évoquer la manière dont Gaudry étudie les objets paléontologiques en sa possession. $\mathrm{La}$ destinée des fossiles mis au jour au mont Luberon, du 19e siècle jusqu'à aujourd'hui, est ensuite abordée en mettant l'accent sur le travail d'expertise, catalogage et restauration effectué récemment sur la collection ainsi rassemblée pour la première fois. À l'aide de cet exemple, on conclut sur ce que devrait être à notre sens la nature des efforts portés sur la collection de Paléontologie du Muséum national d'Histoire naturelle.

\section{MATÉRIEL ET MÉTHODES}

La collection des vertébrés fossiles du mont Luberon du Muséum porte le sigle LUB (aujourd'hui 988 numéros) qui se présente sous deux formes. La plus ancienne est «LUB et numéro », l'actuelle est " MNHN.F.LUB et numéro " inscrite sur les spécimens sur lesquels seul un numéro de millésime était écrit, voire sans numéro du tout. De la sorte la collection cataloguée est en accord avec l'acronyme généralisé de la collection de paléontologie (MNHN pour Muséum national d'Histoire naturelle et $\mathrm{F}$ pour " Fossile »). La collection du Luberon correspond à différents numéros de catalogue d'entrée et millésimes : cat. 64, 1863-1 (Gaudry) ; cat. 146, 1866-6 (Saporta) ; cat. 156, 1866-16 (Gaudry), cat. 790, 1890-25 (Depéret). Sur le plan historique il est à noter que la rédaction de ces catalogues d'entrée n'est pas informative à l'échelle des spécimens mais certains d'entre eux appartenant au cat. 64 sont repris à la fois dans les cat. 146 et 156 . L'expertise de la collection (vérification, restauration) a été effectuée dans le cadre d'une mise à jour globale de la conservation des faunes de mammiferes fossiles du Miocène supérieur initié par l'un d'entre nous (PT) en raison de leur importance dans l'émergence d'une paléontologie pleinement évolutionniste. Le matériel d'origine a été contrôlé relativement à la monographie de Gaudry et al. (1873). Le catalogue numérisé mis à jour est résumé dans les annexes 1 et 2 ; la liste faunique est actualisée dans l'annexe 3.

\section{Histoire DE LA COLLECTION}

\section{L'origine de la collection}

Gaudry n'est pas parti de rien. Avant lui, le gisement, découvert par Christol (1832), a été souvent visité. À ce propos, Gaudry (1873: 6) cite Paul Gervais qui, vingt ans plus tôt, fait un premier bilan : "Les fouilles que MM. De Christol, Piaget, Matheron, Requien, Eugène Raspail, Jourdan, Bravard, Pomel et Caire y ont successivement pratiquées, et celles que j'y ai faites moi-même, ont répandu dans les collections un assez grand nombre d'ossements". À cette liste, Gaudry ajoute le nom de Lartet qui " a remis au laboratoire de paléontologie du Muséum des planches lithographiées qui portent la signature de Bravard " (restées inédites).

Selon Gaudry, outre les spécimens issus de ses propres découvertes, la collection du Muséum inclut des fossiles découverts par l'abbé Caire.

\section{Le travail de Gaudry}

Gaudry (1873: 7) déclare : "[les] premières fouilles remontent à 1862 ; c'est seulement en 1866 que j'ai commencé une exploitation méthodique ". La liste faunique des vertébrés du Luberon établie par Gaudry (1873: 11) compte seize espèces. Elle est ici reproduite en respectant la formulation et l'orthographe originales: "Machaorodus cultridens, Hyana eximia, Ictitherium hipparionum, Ictitherium Orbignyi ?, Dinotherium giganteum, Rhinoceros Schleiermacheri, Acerotherium incisivum ?, Hipparion gracile, Sus major, Helladotherium Duvernoyi, Tragocerus amaltheus, Gazella deperdita, Paleoceras 
MONT LÉBERON.

Machærodus cultridens.
Hyæna eximia.
lctitherium hipparionum.
Ictitherium Orbignyi?
Dinotherium giganteum.
Acerotherium incisivum?
Rhinoceros Schleiermacheri.
Hipparion gracile.
Sus major (c'est pent-être
une race du Sus eryman-
thius).
Helladotherium Duvernoyi.
Tragocerus amaltheus.
Gazella deperdita.
Cervus Matheronis,

PIKERMJ.

Machærodus cultridens. Hyæna eximia.

Ictitherium hipparionum. Ictitherium Orbignyi. Dinotherium giganteum. Acerotherium incisivum? Variétć assez éloignée du $R h$. Schleiermacheri.

Hipparion gracile.

Sus erymanthius.

Helladotherium Duvernoyi. Tragocerus amaltheus. Gazella deperdita.
BALTAVAR.

Machærodus cultridens. Hyæena eximia.

w

$n$

Dinotherium.

$»$

)

Hipparion gracile.

Sus crymanthius ou major.

Helladotherium Duvernoyi. Tragocerus amaltheus. Gazella deperdita.
CONGUD.

$"$
Hyæna eximia.
$"$
$"$
$"$
$"$
$"$

Hipparion gracile.

Tragocerus amaltheus. Gazella deperdita. Cervus Matheronis?

FIG. 2. - Comparaison des listes fauniques des grands gisements du Miocène supérieur (reproduit de Gaudry 1873: 77).

Lindermayeri?, Cervus Matheronis, Testudo de dimension gigantesque, Testudo de taille moyenne "; soit, dans l'ordre, quatre carnivores, un proboscidien, trois périssodactyles, six artiodactyles et deux chéloniens. La liste mise à jour est en Annexe 3.

Afin d'étudier cette faune, Gaudry (1873: 7) a étudié à fins de comparaisons les collections du British Museum (Natural History) à Londres, aujourd'hui Natural History Museum, celles de l'École des Mines à Paris, des musées de Montpellier, de Lyon, d'Aix et du musée Requien à Avignon.

\section{Biostratigraphie, paléobiogéographie}

Le but de Gaudry, déjà explicité à l'occasion de son travail sur la faune de Pikermi, est de " travailler à apprendre si les espèces fossiles ont été fixes ou variables " (Gaudry 1873: 75). Gaudry est l'un des rares évolutionnistes de son temps ; il ne craint pas de faire référence à Darwin et à l'Origine des espèces dans sa monographie sur Pikermi (Gaudry 1866, 1862-1867) et d'affirmer sa conviction sur les notions d' "intermédiaire ", d' "enchaînement ", de " transition » et de " filiation " (Tassy 2006). La faune du Luberon dont il perçoit rapidement la proximité, sinon l'identité, par rapport à celle de Pikermi, lui donne la possibilité d'évaluer la variation du double point de vue stratigraphique et géographique « car, en possédant un grand nom- bre d'os des mêmes espèces, je pourrais connaître si ces espèces ont été des entités immuables, ou bien si elles ont témoigné assez de plasticité pour faire supposer qu'elles sont descendues les unes des autres " (Gaudry 1873: 75, 76). Gaudry soumet au gril de ses observations anatomiques les objections à la filiation, objections alors largement partagées dans la communauté des professeurs du Muséum (Gaudry n'est titulaire de la chaire de Paléontologie que depuis 1872).

Gaudry compare des faunes de gisements proches sur le plan chronologique et est amené à subdiviser le Miocène en deux sous-étages, le plus récent incluant des faunes jugées contemporaines en Espagne, en France, en Hongrie et en Grèce, respectivement Concud, Luberon, Baltavar et Pikermi (Fig. 2) et le plus ancien étant représenté en Allemagne par le gisement d'Eppelsheim (Fig. 3). Ces deux sousétages correspondent au Vallésien et au Turolien des auteurs modernes. Ces travaux l'amènent à extrapoler sa méthode à l'ensemble du Miocène et, au-delà, du Tertiaire (Fig. 4).

Gaudry insiste par ailleurs sur l'aspect paléogéographique et paléoenvironnemental dans la distribution des espèces et le rôle des dispersions. "Le paléontologue qui ne croit pas aux migrations et aux extinctions locales cherchera vainement les enchaînements des êtres anciens " (Gaudry 1873: 


\section{FOSSILES GARACTÉRISTIQUES \\ D'EPPELSHEIN.}

FOSSILES GARACTÉRISTIQUES. DU LÉBERON OU DE PIKERMI.

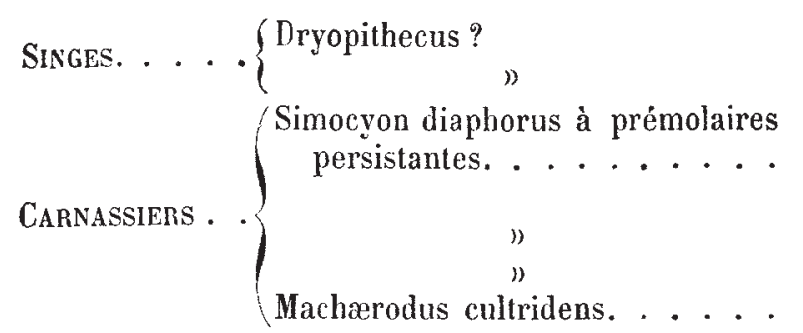

Édentés. . . $\left\{\begin{array}{c}\text { Macrotherium (sp. nova). } \\ \text {, }\end{array}\right.$

Proboscidiens. $\left\{\begin{array}{c}\text { Dinotherium giganteum } \cdots \\ \text { Mastodon longirostris. } \\ " \\ "\end{array}\right.$

PACHYDERMES. $\left\{\begin{array}{l}\text { Rhinoceros Schlciermacheri. . . } \\ \text { Acerotherium incisivum. . . } \\ \text { Chalicotherium. . . . . } \\ \text { Tapirus. } \\ \text { Sus palieochnerus. } \\ \text { Sus antediluvianus. } \\ \text { Sus antiquus. } \\ \text { Hipparion gracile....... }\end{array}\right.$
Mesopithecus.

Simocyon diaphorus à prémolaires en partie caduques.

Hyæna.

Ictitherium.

Machærodus cultridens.

Ancylotherium.

Dinotherium giganteum?

Mastodon Pentelici.

Mastodon turicensis.

Rhinoceros Schleiermacheri?

Rhinoceros pachygnathus.

Acerotherium incisivum?

Leptodon.

Chalicotherium.

Sus erymanthius et major.

Hipparion gracile.

Helladotherium.

Camelopardalis.

Palæotragus.

Palæoryx.

Palæoreas.

Tragocerus.

Antidorcas.

$\begin{array}{ll}\text { ") } & \text { Gazella. } \\ & \text { Dremotherium }\end{array}$

$\begin{array}{ll}n & \text { Gazella. } \\ & \text { Dremotherium. }\end{array}$

Dorcatherium.

Cervus anocerus, dicranocerus. .

)

FIG. 3. - Essai de subdivision du Miocène supérieur à partir de la comparaison des faunes d'Eppelsheim (aujourd'hui datée du Vallésien) et du Luberon et Pikermi (aujourd'hui datée du Turolien) (reproduit d'après Gaudry [1873: 84]). 


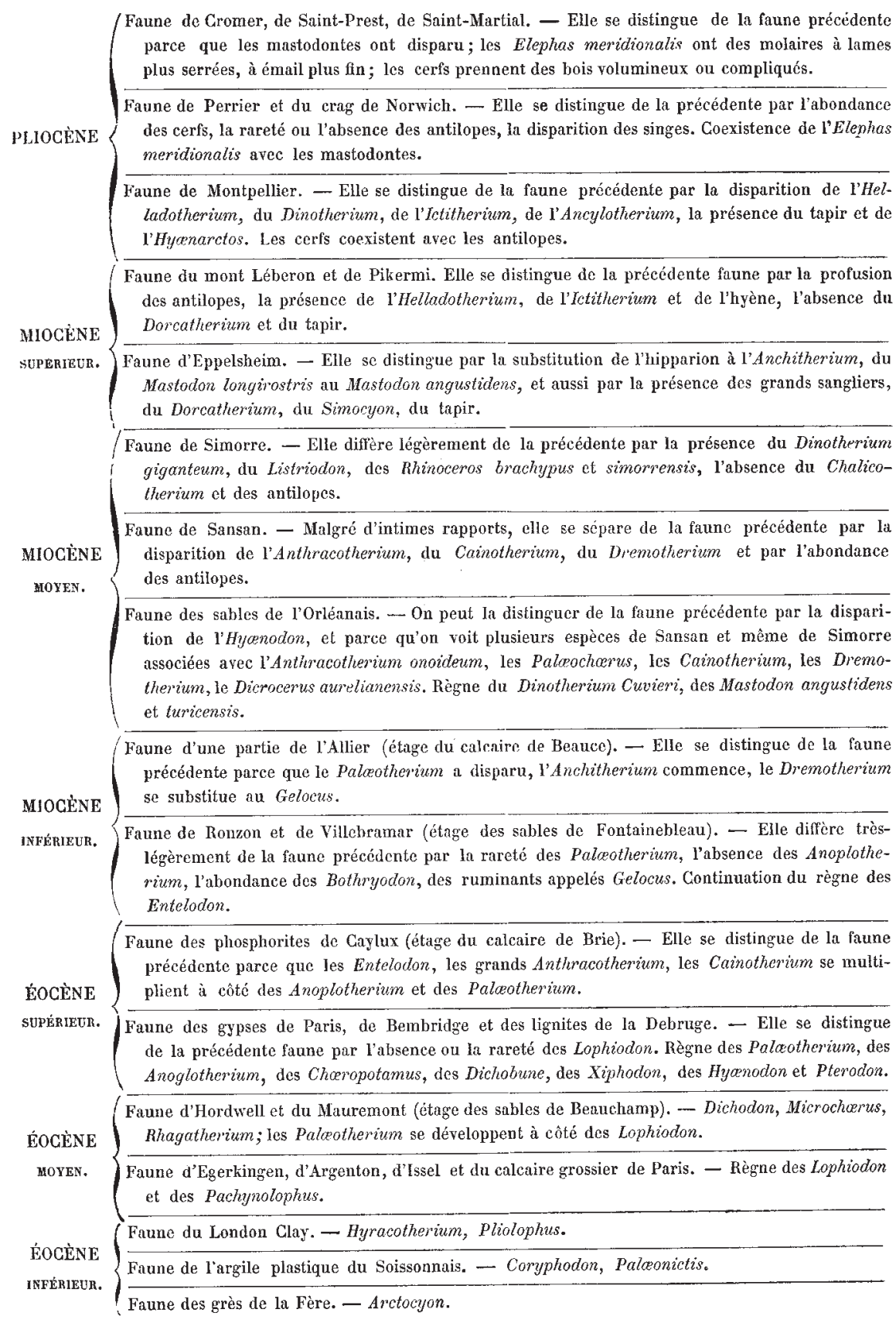

FIG. 4. - Succession des faunes continentales au Tertiaire selon Gaudry (1873: 86). 
88). Il est vrai que dans sa démonstration du rôle des environnements locaux Gaudry tend à considérer les archives paléontologiques comme fiables et non aléatoires, ce en quoi sa prudence pourtant proverbiale sera facilement mise en défaut par les découvertes futures. Cette restriction est toutefois de peu de portée vis-à-vis de la problématique évolutionniste de Gaudry. La faune du Luberon est une nouvelle opportunité pour questionner la relation entre l'évolution des espèces et leur position stratigraphique, relation qui a toujours été au centre des préoccupations de Gaudry. Ce dernier pose ainsi le problème : "Si la plupart des naturalistes sont d'accord sur l'utilité de l'étude des fossiles, ils ne le sont pas encore sur la méthode paléontologique qu'ils doivent employer (...) Alors il faut chercher s'il n'y a pas quelque méthode rationnelle pour fixer l'âge des fossiles, et ceci conduit forcément les paléontologues à examiner la doctrine de l'évolution. Plusieurs personnes pensent que la discussion de cette doctrine a seulement un intérêt philosophique : " je ne le crois pas ; il me semble que nulle question n'importe davantage à la géologie "pratique" "(Gaudry 1869: 45 ; ce texte est repris dans Gaudry 1888).

\section{Évolution}

Une preuve de la réalité de la notion de filiation est tirée par Gaudry du concept d'espèce analogue hérité de Lamarck, appliqué aux mammifères fossiles miocènes du Luberon. Ce faisant, tout au long de ses démonstrations, Gaudry évoque le plan de la création, la Création, le Créateur, l'Auteur du monde, ou, très clairement, Dieu. Ces figures de style chez un penseur chrétien ont été largement responsables d'une réelle tendance à ne pas tenir Gaudry pour un authentique évolutionniste et encore moins un darwinien (Conry 1987: 54). On peut y voir aussi une rhétorique obligée, destinée à faire mieux passer des idées évolutionnistes dans un milieu hostile. Aussi peut-on lire à propos des «formes analogues » : "Ces analogues révèlent une certaine ressemblance entre la faune du miocène supérieur et les faunes qui l'ont précédée ou suivie. Quoique cette ressemblance se manifeste souvent dans les traits généraux plutôt que dans les détails, elle doit être prise en considération par les hommes qui cherchent à comprendre le plan de la création. En effet, ou bien elle force à admettre ce qu'on a appelé la loi d'imitation, c'est-à-dire à supposer qu'en créant les êtres d'une époque géologique, Dieu a pris en partie pour modèles les êtres des époques précédentes, ou bien il faut croire que les analogies représentent des liens de parenté soit proche, soit éloignée " (Gaudry 1873: 91). La conclusion qu'en tire Gaudry est sans ambiguïté : "Je préfère la seconde hypothèse, parce que la plupart des espèces analogues ont une si forte ressemblance comparativement à celle des différences, qu'il paraît avoir été plus simple de les tirer les unes des autres " (Gaudry 1873: 91). Au final, Gaudry rappelle l'effet sur la paléontologie du début du 19 e siècle - effet qu'on peut aujourd'hui juger désastreux - de l'interprétation par Cuvier des momies d'Égypte et de l'erreur concomitante sur la notion de durée en géologie. La métaphysique de Gaudry et, peut-être, sa volonté de ne pas froisser les croyants, ne sont pourtant jamais loin surtout lorsqu'à propos des mécanismes de l'évolution sont citées concurrence vitale, sélection naturelle, influences du milieu, migration mais aussi un "principe (qui) a résidé dans une région supérieure, trop haute pour que nous puissions, quant à présent, le bien saisir " (Gaudry 1890: 296), principe qualifié de "peu darwinien " par un exégète pourtant sensible au spiritualisme de l'auteur des Vertébrés fossiles $d u$ Mont Léberon (Durand 1975: 228).

Pour en revenir au travail sur la faune du Luberon, partant du travail de Darwin sur les « races naturelles vivantes », Gaudry (1873: 92) dans le paragraphe 6 de la monographie intitulé "Sur la distinction des races et des espèces de mammifères à la fin des temps miocènes ", essaie de faire la part de la race (ou variété) et de l'espèce dans la dimension paléontologique, tâche délicate s'il en est. Sa solution, à la fois élégante et profonde, est remarquablement moderne. Il l'illustre notamment par plusieurs exemples, équidés, ruminants, carnivores. Citons celui des équidés (Figs 5; 6). "Ainsi les divers hipparions de Pikermi et du Léberon, qui ne se distinguent guère les uns des autres que par leurs formes plus lourdes ou plus grêles, ont pu être des races d'une même espèce. Au contraire, lorsque les caractères qui séparent les animaux semblent indiquer une différence dans leur degré 

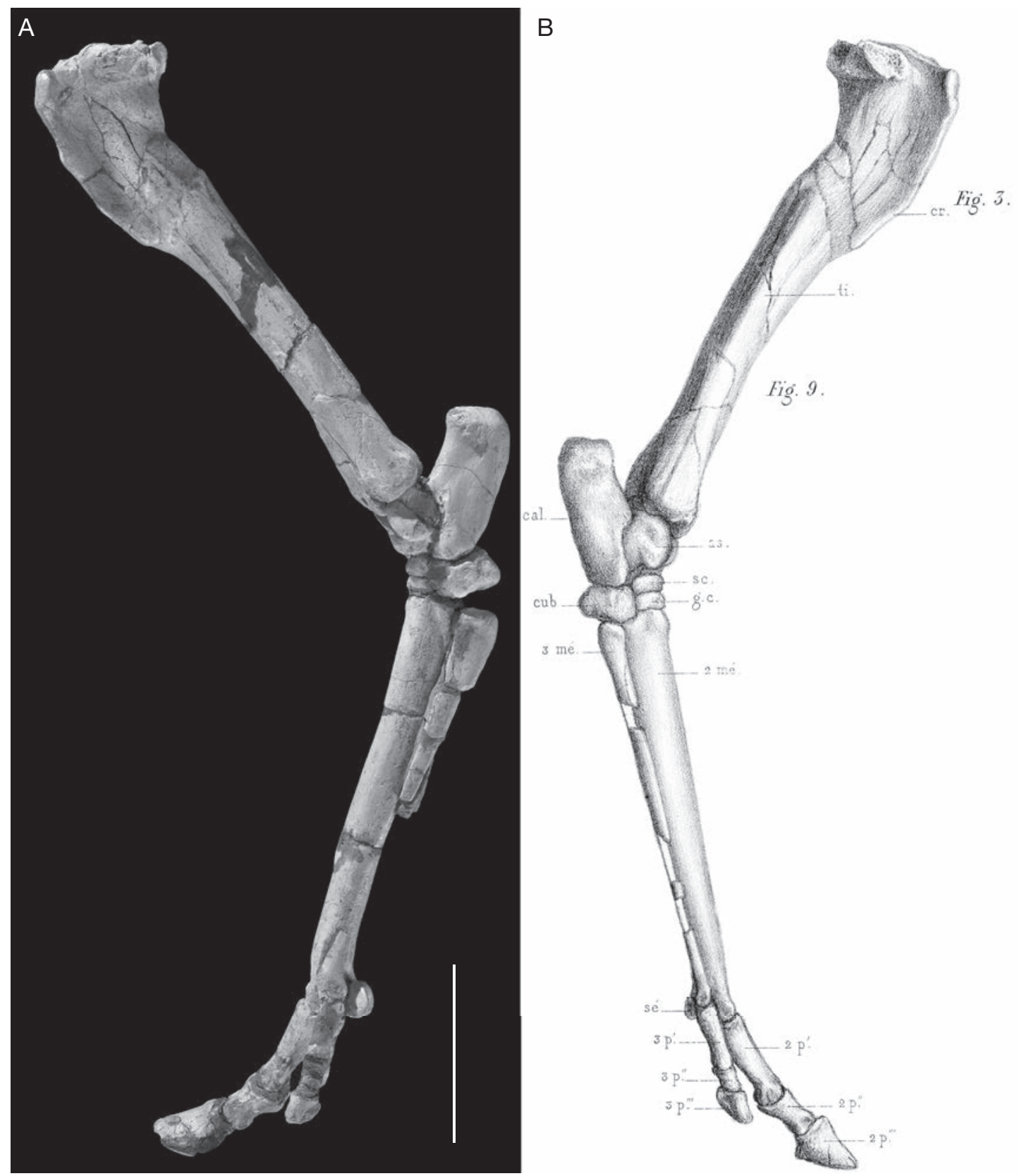

Fig. 9 .

FIG. 5. - Hipparion prostylum Gervais, 1849, membre postérieur gauche, MNHN.F.LUB918, Luberon (Miocène supérieur) : A, vue latérale ; B, idem, reproduit de Gaudry (1873: pl. 6, fig. 9). Cliché L. Cazes (MNHN). Échelle : $10 \mathrm{~cm}$.

d'évolution, on doit supposer que ces animaux sont devenus des espèces distinctes, c'est-à-dire qu'ils ont cessé de donner ensemble des produits féconds ; car, s’il en eût été autrement, la nature aurait tourné dans le même cercle, au lieu de présenter ces divergences qui ont imprimé à chaque 
époque géologique une physionomie particulière " (Gaudry 1873: 97).

On ne saurait mieux introduire la notion d'espèce biologique dans la dimension temporelle par le biais d'une analyse purement morphologique.

Sur le plan de l'analyse des caractères, Gaudry reconnaît l'association de caractères primitifs et évolués chez une même espèce, autrement dit, l'évolution en mosaïque de De Beer (1954) ou l'hétérobathmie de Takhtajan reprise par Hennig (1965), c'est-à-dire le fait que divers organes n'évoluent pas nécessairement de concert. Le cas des hipparions peut être choisi comme exemple. "Les remarques qui ont été faites par plusieurs naturalistes (...) et par moi m'ont porté à penser que sans doute le genre cheval est descendu du genre hipparion. En entreprenant des fouilles dans le mont Léberon, j'avais l'espérance que la comparaison des nombreux os de ce gisement et de celui de Pikermi fournirait quelques nouveaux indices de transition entre ces deux genres" (Gaudry 1873: 37). Si Gaudry avoue avoir été déçu dans ce domaine, il trouve des caractères transitoires sur d'autres espèces notamment nord-américaines et du sous-continent indien. L'espèce des Siwaliks Hipparion antilopinum Falconer \& Cautley, 1849 dont les doigts latéraux sont plus régressés que ceux des espèces de Pikermi et du Luberon, à la manière des espèces du genre Equus, lui paraît exemplaire : "l'Hipparion antelopinum était un équidé qui avait perdu dans ses pattes le caractère de son ancêtre l'hipparion, bien qu'il l'eût retenu encore dans sa dentition " (Gaudry 1873: 40). En outre, cette phrase renvoie à une note de bas de page où Gaudry aborde finement les conséquences nomenclaturales de ces partages de caractères, malheureusement en privilégiant le rôle prééminent des caractères primitifs : "Si ma supposition se vérifiait, quelques personnes seraient sans doute disposées à proposer un nouveau nom de genre pour un animal qui aurait eu des pattes de cheval avec une dentition d'hipparion. Il me semble pourtant préférable de conserver le nom d'hipparions aux animaux qui sont en voie de prendre la forme Equus jusqu'au moment où ils ont réalisé complètement le type de ce genre. En paléontologie, les noms d'espèces doivent autant que possible refléter les dégradations des formes interposées entre les espèces à caractères bien accusés qui constituent les types des genres " (Gaudry 1873: 40, note 2). On ne pourra donc pas tenir Gaudry pour un précurseur de la cladistique malgré son analyse de caractères. En dernier lieu, on sait désormais que les caractères dérivés partagés par les espèces d'Equus et certaines espèces d'hipparions ne sont pas des synapomorphies mais des homoplasies. Mais ce résultat importe peu, c'est bien entendu ici la méthode analytique qui compte, pas le résultat (toujours provisoire de surcroît).

La partie consacrée aux invertébrés est tout aussi évolutionniste. Fischer \& Tournouër (1873: 163) après avoir décrit les fossiles du Luberon (lamellibranches et gastéropodes; à quoi s'ajoutent des bryozoaires et polypiers du gisement voisin de Cabrières-d'Aigues) dans un langage plus proche de la taxinomie traditionnelle, ne craignent pas d'affirmer suite à leur étude de la variabilité des formes au Miocène : "Cette variabilité n'est pas en contradiction avec l'hypothèse de la filiation des espèces par voie de transformation. Elle en est au contraire l'expression et la condition nécessaire; il faut bien que les types varient pour qu'ils se transforment $"$.

Ainsi avec les Animaux fossiles du Mont Léberon (Vaucluse), la paléontologie moderne est née, assurément, avec ses facettes évolutives, biostratigraphique et paléobiogéographique. Pour rester dans le domaine du Miocène supérieur, un article représentatif de la biochronologie telle qu' elle est pratiquée aujourd'hui (Bernor et al. 1996) s'inscrit dans le fond et dans la forme dans la continuité de Gaudry (1873). Le travail novateur de Gaudry, dans la droite ligne de son étude des mammifères de Pikermi, doit donc tout à une collection issue de recherches sur le terrain initiée dans le but de comprendre l'évolution des espèces dans la dimension chronologique.

\section{Taphonomie, muséologie}

Le souci pédagogique de Gaudry s'est aussi appliqué à la taphonomie. La première planche de sa monographie représente un exemplaire de ce que l'on appelle aujourd'hui un bone bed, un bloc de sédiment riche en ossements appartenant à diverses espèces, "dans le but, explique Gaudry (1873, légende de la planche I), de présenter un exem- 


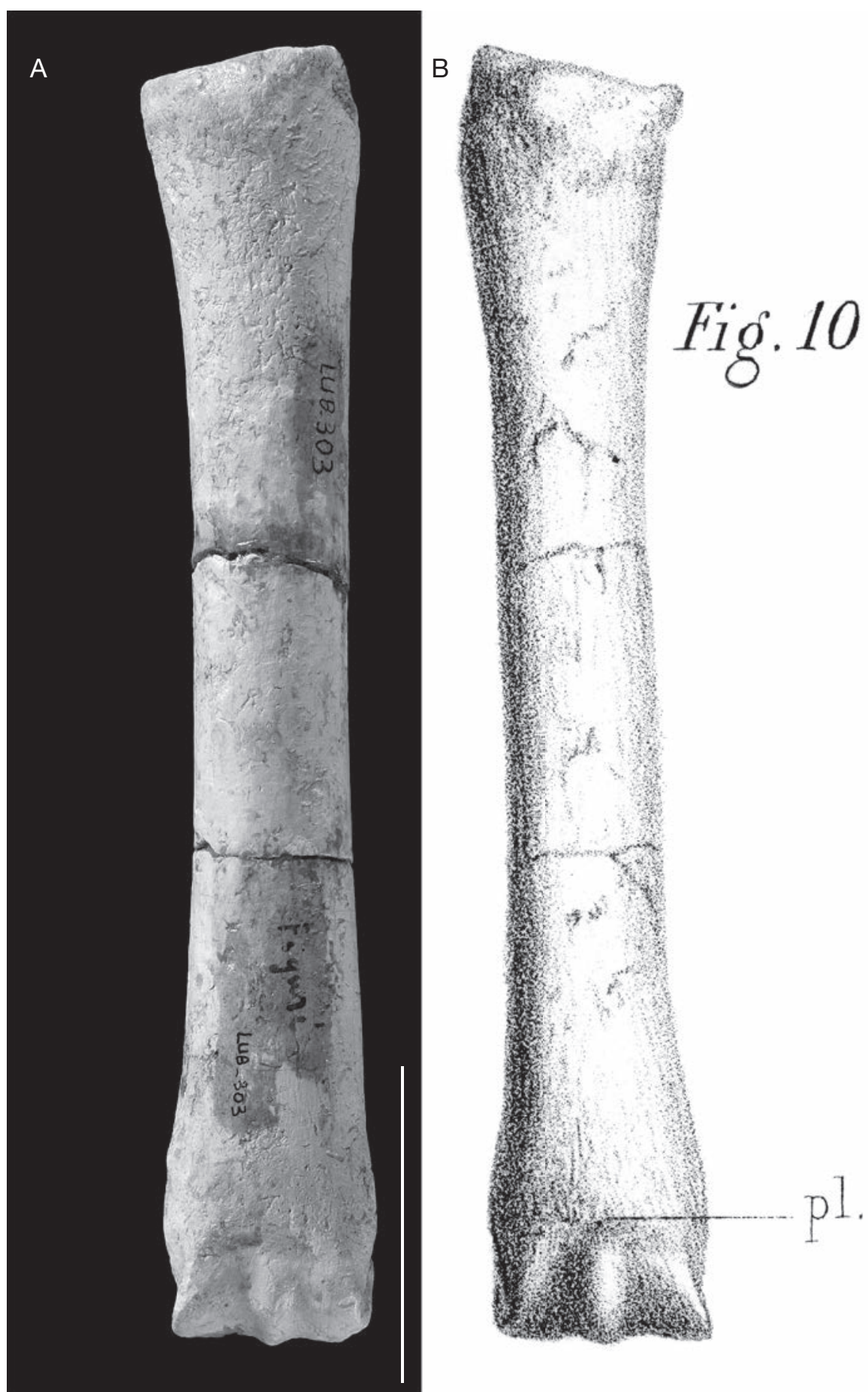

FIG. 6. - Hipparion prostylum Gervais, 1849, troisième métacarpien gauche MNHN.F.LUB303, Luberon (Miocène supérieur): A, vue antérieure ; B, idem, reproduit de Gaudry (1873: pl. 6, fig. 10). Cliché L. Cazes (MNHN). Échelle : $5 \mathrm{~cm}$. 
ple du mode d'enfouissement des os ". Ce bloc, longtemps conservé au sein de la collection de géologie du MNHN, est aujourd'hui exposé avec son pittoresque cartel d'origine dans la galerie de paléontologie du Muséum. Depuis la figuration par Gaudry, deux crânes d'Hipparion prostylum ont été ajoutés au bloc ossifere sans que l'on puisse dater cette adjonction, même si l'on peut présumer que cette initiative est due à Gaudry lui-même (Fig. 7).

Gaudry (1873: 9) est moins loquace sur l'aspect muséal de son travail. Il explique cependant que le matériel qu'il a mis au jour est conséquent : «Les os que j'ai recueillis sont au nombre d'environ 1200 ; je les ai donnés au Muséum de Paris ». L'une des raisons de ce laconisme est peut-être liée au statut des fossiles au sein de la collection du Muséum, sujet d'intenses frictions au sein de l'établissement depuis la création de la chaire de paléontologie en 1853. Les fossiles n'étaient alors pas régis par la chaire de paléontologie mais par les différentes chaires à collection, notamment l'anatomie comparée et la géologie. Ce n'est qu’à partir de 1879 que Gaudry put rassembler et organiser les fossiles conservés au Muséum, et particulièrement ses propres collections, dont celle du Luberon, prélude à sa création, en 1898, du bâtiment de paléontologie actuel.

Un dernier point est à citer concernant la manipulation des objets. Une fois dégagé de sa gangue, le fossile doit-il être sacralisé ou bien peut-il être l'objet d'interventions à des fins de recherche? Cette question est d'autant plus d'actualité que la loi du 4 janvier 2002 dite " loi Musée " s'applique aussi aux objets naturels, y compris les fossiles. Gaudry s'était déjà posé la question et y a répondu en scientifique. À propos des équidés du genre Hipparion, soucieux de découvrir le niveau évolutif de la dentition et la morphologie de la " colonnette antérieure externe » des molaires, Gaudry (1873: 34, pl. 6) retire le cément qui recouvre la couronne : le spécimen devient alors porteur d'un message scientifique (Fig. 8). La « colonnette " telle qu'on peut l'observer aujourd'hui est plus courte que sur le dessin de Gaudry (Fig. 8B), la cassure est sans doute postérieure à la figuration par Gaudry. Il est possible aussi que la hauteur d'origine ait été reconstituée par Gaudry lui-même. En effet, le style artistique des planches de la monographie du Luberon laisse souvent à penser que les fossiles sont mieux conservés qu’ils ne l'étaient en réalité. Il n'en reste pas moins vrai que la dissection opérée par Gaudry montre qu'ici le fossile est conçu comme un objet à la fois naturel et scientifique, paradoxe ontologique digne d'intérêt.

Un dernier mot sur le style : Gaudry ne craint pas d'associer dans un même paragraphe poésie, réflexions sur la règle d'antériorité en systématique et anatomie des moulages naturels endocrâniens. La section consacrée à Gazella deperdita (Gervais, 1847) débute ainsi : «J'ai trouvé au pied du Léberon les débris de quatre-vingt-dix gazelles; sans doute, ces animaux ont contribué beaucoup à embellir les paysages de la Provence " (Gaudry 1873: 57).

\section{La collection hier}

Au cours du 20e siècle, la collection des vertébrés fossiles du Luberon a été souvent visitée, étudiée, le plus souvent à l'occasion de comparaisons avec de nouvelles découvertes de faunes miocènes mais parfois à l'occasion de la révision même d'une des espèces du Luberon comme Gazella deperdita (Heintz 1969, 1971 ; Bouvrain 1977). On peut citer aussi différentes révisions des équidés, la systématique des hipparions étant un défi sans cesse renouvelé (Sondaar 1974 ; Skinner \& MacFadden 1977 ; MacFadden 1980 ; Bernor et al. 1980 ; Woodburne \& Bernor 1980) mais aussi les suidés (Bonis \& Bouvrain 1996), les hyénidés (Howell \& Petter 1985) ou encore les moulages endocrâniens des bovidés (Dechaseaux 1961). Cependant, aussi étonnant que cela puisse paraître, aucun paléontologue n'a jamais pu voir la collection dans son intégralité et son intégrité. Pire, l'état de cette collection fondatrice au seuil du 21 e siècle était accablant. Si de nombreux figurés de Gaudry (1873) étaient bien accessibles, tous ne l'étaient pas. Certains étaient dans un état déplorable comme si une catastrophe naturelle s'était abattue sur la collection. Effets de l'inondation due à la crue de la Seine de 1910 ? Aucune archive ne le précise. Par ailleurs, la préparation de certains spécimens n'était même pas achevée et ce, depuis la parution même de la monographie, y compris pour des fossiles figurés par Gaudry. Il convenait de conjurer ce sort aussi funeste qu'immérité.

Le $20^{e}$ siècle, et spécialement l'après-guerre, voit en France l'essor de la recherche comme activité 


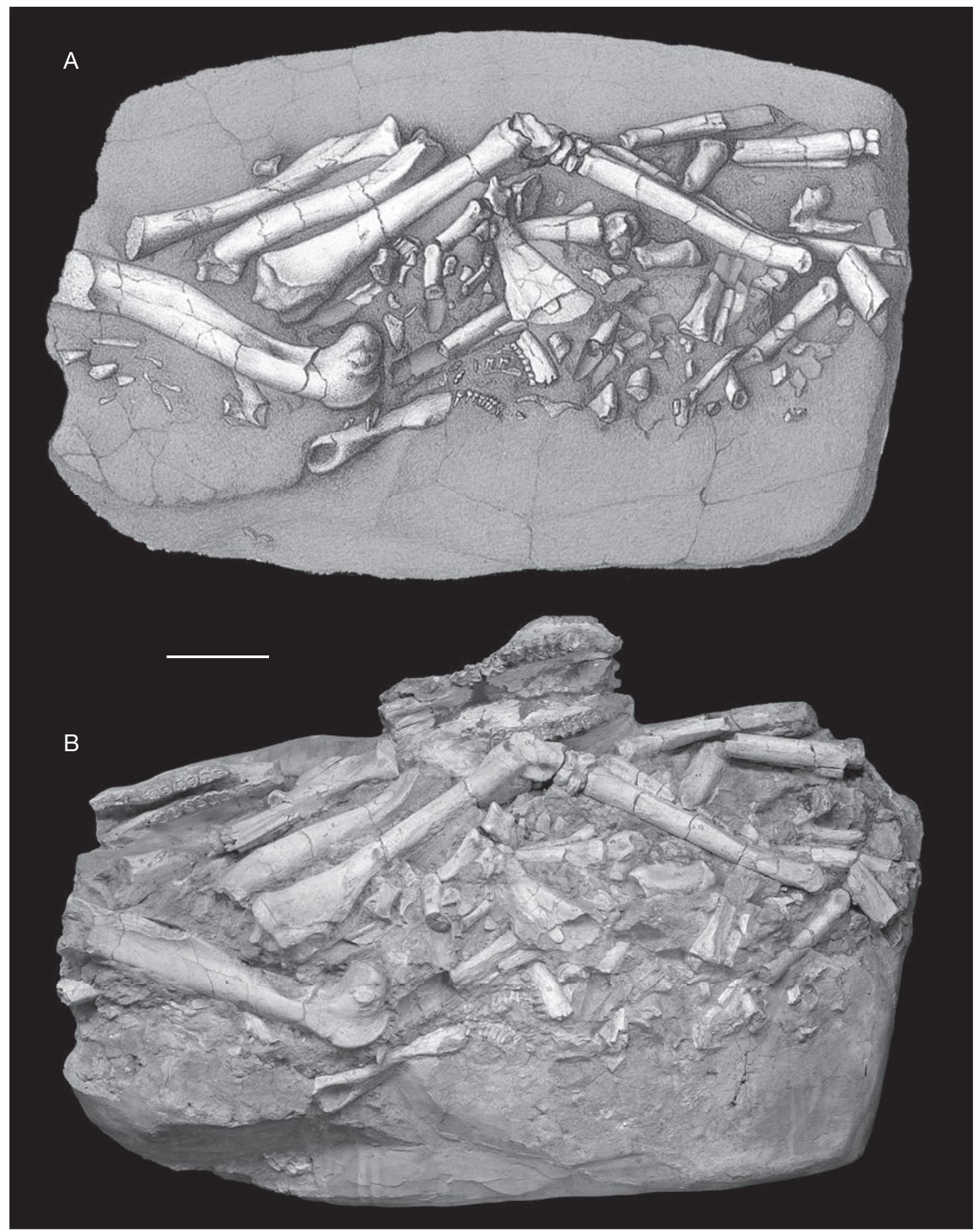

FIG. 7. - Bloc ossifère du Luberon MNHN.F.LUB987 (Miocène supérieur) : A, reproduit de Gaudry (1873: pl. 1) et inversé ; B, tel qu'il est exposé dans la Galerie de Paléontologie du Muséum national d'Histoire naturelle avec ses deux crâne d'Hipparion prostylum. Cliché L. Cazes (MNHN). Échelle : $10 \mathrm{~cm}$. 
sociale privilégiée. Dans ce cadre, le laboratoire de Paléontologie du Muséum se transforme en laboratoire associé au CNRS (L.A.12). La collection de paléontologie est considérée comme un support à la recherche, la notion patrimoniale n'existe pas alors. Parmi les pratiques de conservation de l'époque, la numérotation des spécimens selon un code par localité était l'une des plus répandues. L'usage dans les publications paléontologiques était en effet de privilégier des numéros de catalogue modernes et non des numéros d'entrée de lot telle que pratiquée au Muséum à l'origine. Le code choisi était un sigle de localité, de gisement, souvent composé de trois lettres mais pas toujours, suivi du numéro du spécimen. L'acronyme LUB est celui du Mont Luberon, comme l'acronyme PIK celui de Pikermi. Un spécimen du Luberon porte parfois (mais pas toujours) un numéro d'inventaire (entrée du lot en collection : 156, ce qui correspond au plus gros de la collection Gaudry du Luberon et (toujours, désormais) un numéro de catalogue spécifique : par exemple LUB 7. Curieusement, cette numérotation (qui remonte à une date dont les archives ne font pas mention, sans doute durant les années 1960-70) n'était pas accompagnée d'un catalogue papier ; il y a d'autres exemples semblables, heureusement rares.

\section{La restauration}

Un premier travail de regroupement des spécimens constituant la collection a été réalisé en 2007 lors de vacations (120 h). Ces vacations ont aussi servi à une première phase de conditionnement, d'identification et de dépoussiérage/nettoyage. En effet, au fil des décennies, et a fortiori des siècles, les fines particules de poussière s'agglomèrent sur les ossements surtout s'ils sont recouverts d'une colle ou d'un vernis un tant soit peu poreux. Les os peuvent perdre ainsi leur couleur d'origine, notamment lorsqu'ils sont blancs, comme ceux du Luberon. De la sorte des ossements de divers gisements peuvent prendre, à cause de cette patine séculaire, un même aspect grisâtre, par exemple la symphyse mandibulaire d'Hipparion prostylum (Fig. 9) figurée par Gaudry (1873: pl. 6, fig. 2). Un fossile non numéroté, une fois séparé de son étiquette pour quelque cause fortuite, et le voilà pratiquement anonyme au sein de centaines d'autres sauf à l'œil du spécialiste si par chance ce fossile a été déjà étudié et figuré, et encore cela n'est pas toujours suffisant. Un exemple significatif est fourni par Heintz (1971) qui signale qu'il n'a pu mettre la main sur le crâne de Gazella deperdita figuré par Gaudry (1873: pl. 11, fig. 1) et le considère comme perdu. En réalité ce crâne avait reçu des numéros correspondant au gisement de Maragha : 1908-10 et MAR 1851 - ce qui laisse à penser que l'erreur vient de l'époque du catalogage des fossiles de Maragha de la collection Mecquenem. Mieux encore, les deux cornes étaient cassées. Les pointes ont été retrouvées parmi les restes fragmentaires de Maragha, portant les numéros MAR 2097 et MAR 1130. Aujourd'hui ce crâne a recouvré son intégrité sous le numéro MNHN.F.LUB681 (Fig. 10).

Ce travail a été mené par l'un d'entre nous (VP). La mise à niveau consacrée à cette collection d'un millier de spécimens représente plus de $700 \mathrm{~h}$ en conservation et restauration. Les principales activités qui ont étés menées sont: l'identification taxonomique et anatomique, la restauration (nettoyage, réparation, consolidation), le conditionnement, l'étiquetage, le marquage et l'informatisation des spécimens.

La collection était éparpillée dans le bâtiment de Paléontologie. Les spécimens ont, dans un premier temps, été regroupés dans un unique lieu de rangement (une salle équipé d'armoires compactus). La logique de stockage de la collection de paléontologie - environ six millions de spécimens - est en effet un rangement grandement fondé sur la taille des spécimens (et les mammifères fossiles ne dérogent pas à la règle). Le bâtiment de paléontologie s'étant vite révélé insuffisant pour une telle collection, les meubles se sont accumulés un peu partout selon les opportunités, du sous-sol au balcon ; les objets ont été rangés en fonction de leur taille et de celle des tiroirs, les hors-normes étant placés dans les lieux jugés les plus fiables (les plus éloignés des circulations et manipulations habituelles). On comprendra la difficulté de consultation liée à ce rangement, assez générale en ce qui concerne les vertébrés, la taille des objets d'un même gisement allant du millimètre au mètre.

Le nettoyage des spécimens a obéi à quelques règles simples. Les spécimens ont été dépoussiérés et une 

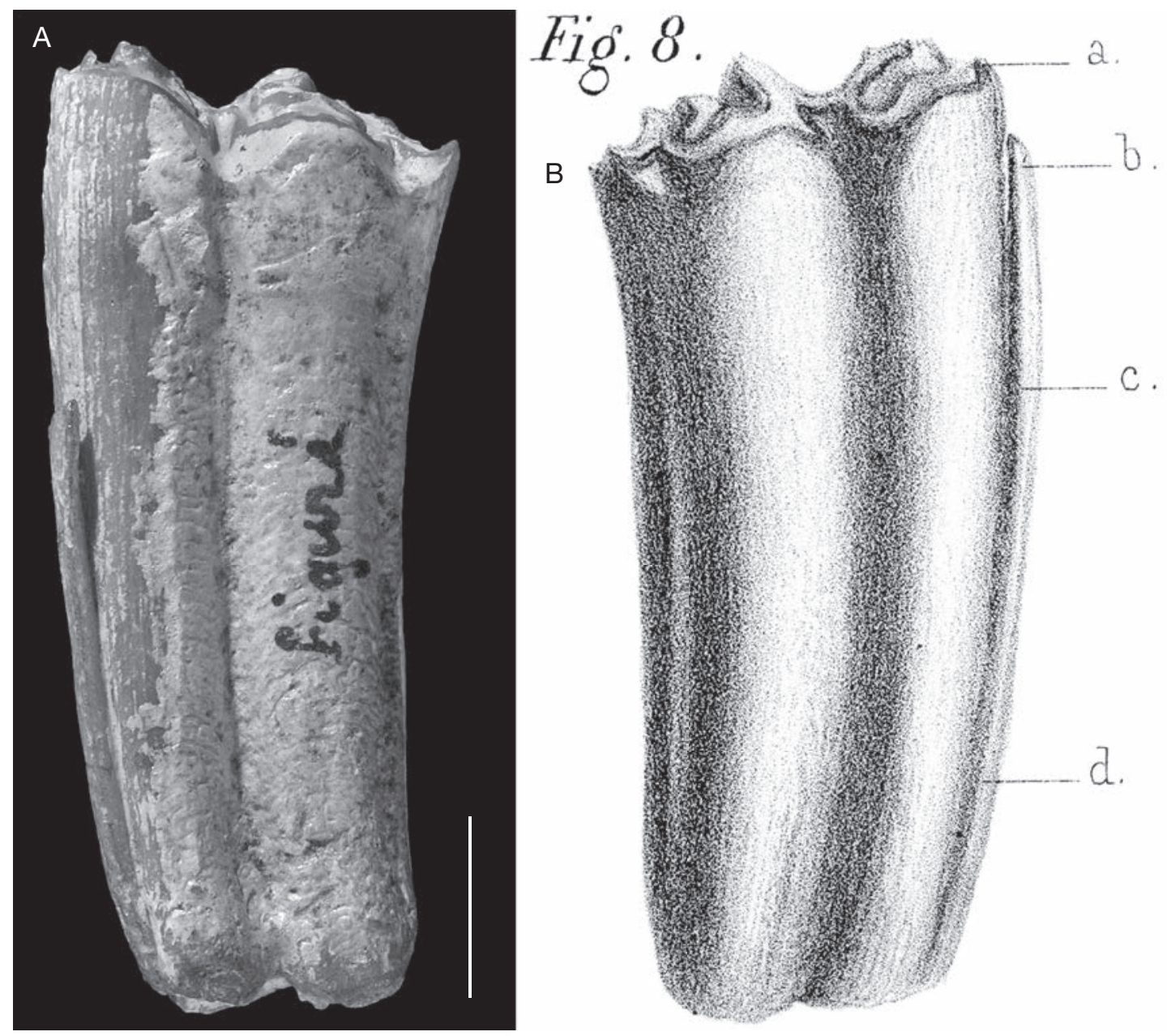

FIG. 8. - Hipparion prostylum Gervais, 1849, m3 gauche MNHN.F.LUB7, Luberon (Miocène supérieur) : A, vue vestibulaire ; B, idem reproduit de Gaudry (1873: pl. 6, fig. 8) ; la colonnette (c.) est plus courte que sur la figure donnée par Gaudry, probablement cassée après l'illustration. Cliché L. Cazes (MNHN). Échelle : $1 \mathrm{~cm}$.

grande partie d'entre eux nettoyés mécaniquement au pinceau à l'eau chaude, parfois à l'acétone. Avant cette intervention certains spécimens avaient déjà subi un nettoyage sommaire et un encollage important. D'autres avaient été encollés sans nettoyage préalable (spécimen consolidé avec sa couche de crasse). Cela explique le nombre de fossiles présentant des restes de colle ancienne (colle dite " animale »), de crasse et une importante couche de colle moderne (de type consolidant acrylique). Le nouveau nettoyage de ces spécimens étant lent et fastidieux (successions de nettoyages mécaniques à l'acétone, de séchages et de nettoyages mécaniques à l'eau chaude), l'objectif a été de remettre rapidement en état ces spécimens sans zèle excessif. Les spécimens remarquables, tels les figurés, ont cependant été nettoyés au mieux.

Les nombreux fossiles détériorés ont été restaurés en utilisant un consolidant acrylique (un polymère de méthyle méthacrylate et n-butyle méthacrylate) dilué dans de l'acétone à différentes concentrations afin d'obtenir la viscosité souhaitée en fonction de l'opération réalisée : collage (concentration 


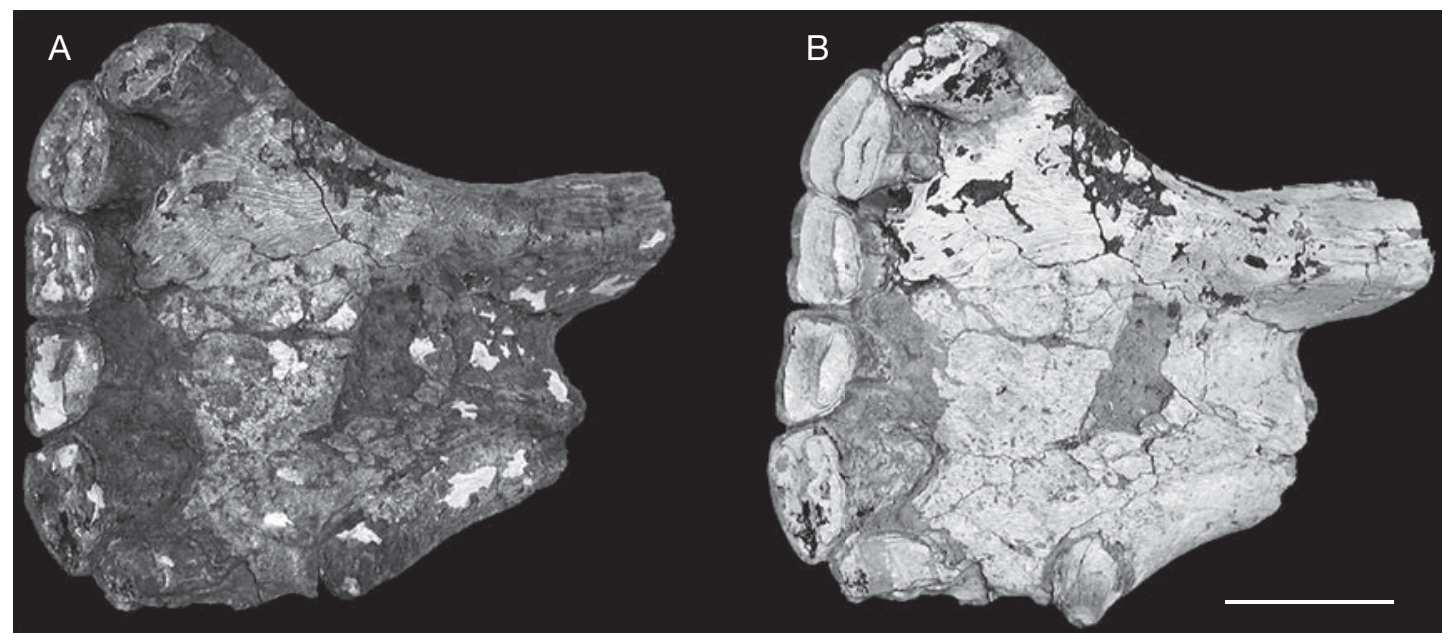

FIG. 9. - Hipparion prostylum Gervais, 1849, symphyse mandibulaire MNHN.F.LUB869, Luberon (Miocène supérieur), vue occlusale : A, avant nettoyage ; $\mathbf{B}$, après nettoyage. Clichés V. Pernègre. Échelle : $2 \mathrm{~cm}$.

environ 50-60\%), consolidation (concentration environ 10-20\%). La recherche d'éléments qui se complètent a été particulièrement longue, en raison de la dissémination originelle de la collection : les éléments constituant un unique spécimen ayant trop souvent été séparés physiquement sans indication. À titre d'exemple, on peut noter que le spécimen le plus dégradé a été retrouvé en sept éléments individualisés. Quelques spécimens présentent encore des manques : l'identification de ces derniers se fonde sur la présence d'amas de cire ou de colle ancienne et/ou de cassures fraîches. Cette restauration permet en retour un contrôle de la fiabilité des illustrations originales. Un exemple est fourni par le tibia MNHN.F.LUB955 (Fig. 11) figuré par Gaudry (1873: pl. 4, fig. 7). Le spécimen était en six morceaux retrouvés dans un tiroir rassemblant des restes fragmentaires de : «? Luberon, ? Sansan, ? Simorre» (Fig. 12). Une fois le tibia reconstitué, l'analyse des cassures montre que des fragments de ce qui restait de la fibula associée ont disparu depuis son étude initiale et que l'extrémité articulaire distale telle qu'on la voit sur le dessin de Gaudry (Fig. 11B) est une interprétation artistique car la gangue occupe toujours la fosse articulaire.

L'identification taxonomique des spécimens a ensuite permis de faire le premier tri de rangement, le second étant leur identification anatomique. La logique retenue pour le rangement de l'ensemble de la collection est celle qui régit la collection de paléontologie des vertébrés du Muséum : regroupement de niveau 1 par gisement, regroupement de niveau 2 par taxon, regroupement de niveau 3 par anatomie. Le matériel retenu pour le conditionnement des spécimens est de quatre sortes : tiroirs bois, cuvettes cartonnées, boîtes plastiques polystyrène cristal et mousse polyéthylène (films et plaques).

Comme dit plus haut, chaque spécimen porte un numéro unique de catalogue qui est reporté physiquement (peinture acrylique blanche et encre de Chine noire). Le spécimen est accompagné d'une étiquette papier portant les principales informations associées : collection, numéro, identifications taxonomique et anatomique, gisement, âge (figuration le cas échéant). Cette étiquette est protégée en sachet zippé polyéthylène.

La totalité de la collection a été soumise à une lecture sous lumière ultra-violette en salle aveugle afin de révéler d'anciens marquages invisibles ou peu discernables en lumière naturelle suite à la dégradation naturelle de l'encre et au vieillissement des fossiles. L'informatisation des spécimens s'est déroulée en concomitance avec les phases de marquage et d'étiquetage. Elle s'est divisée en deux temps : 


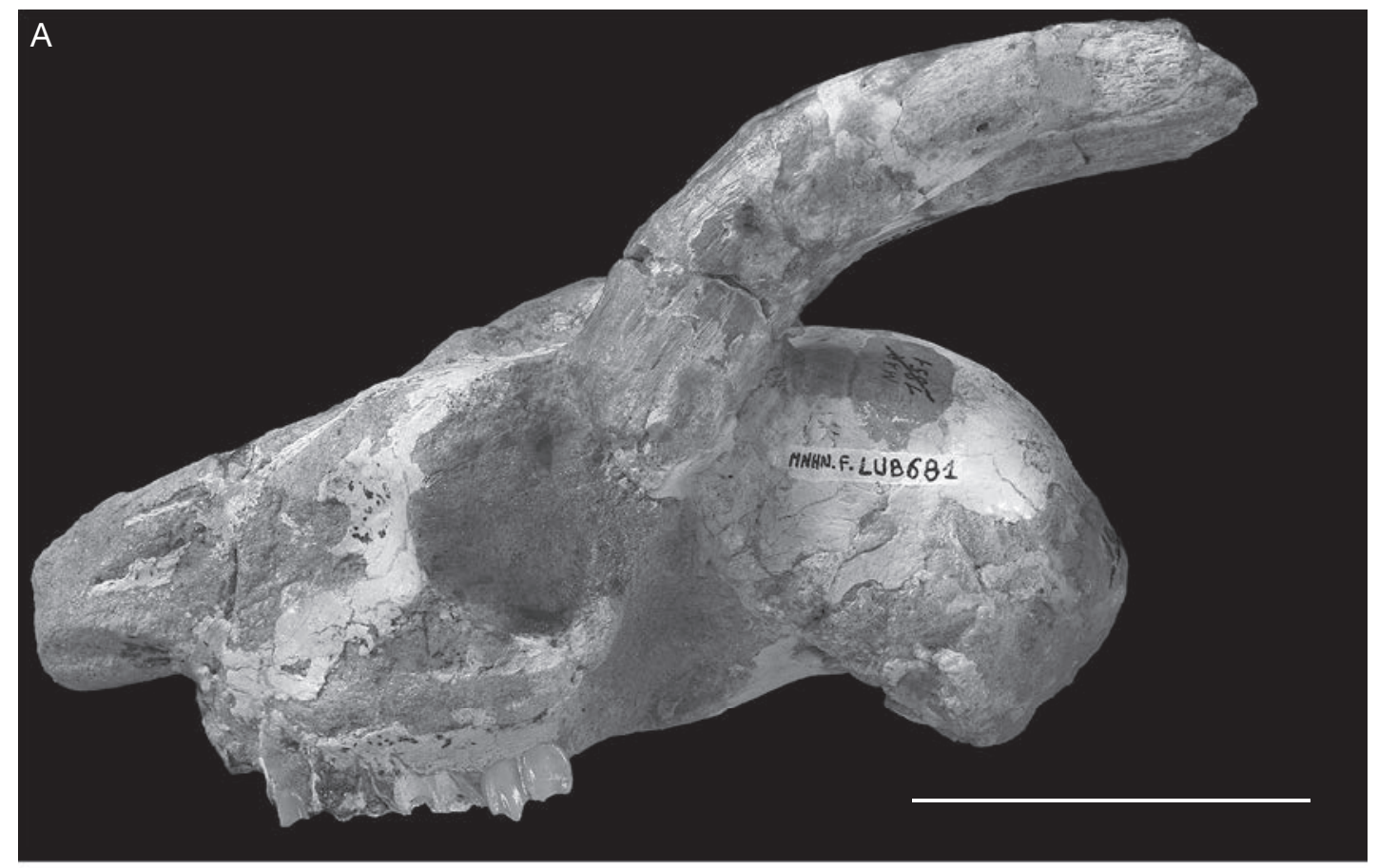

B

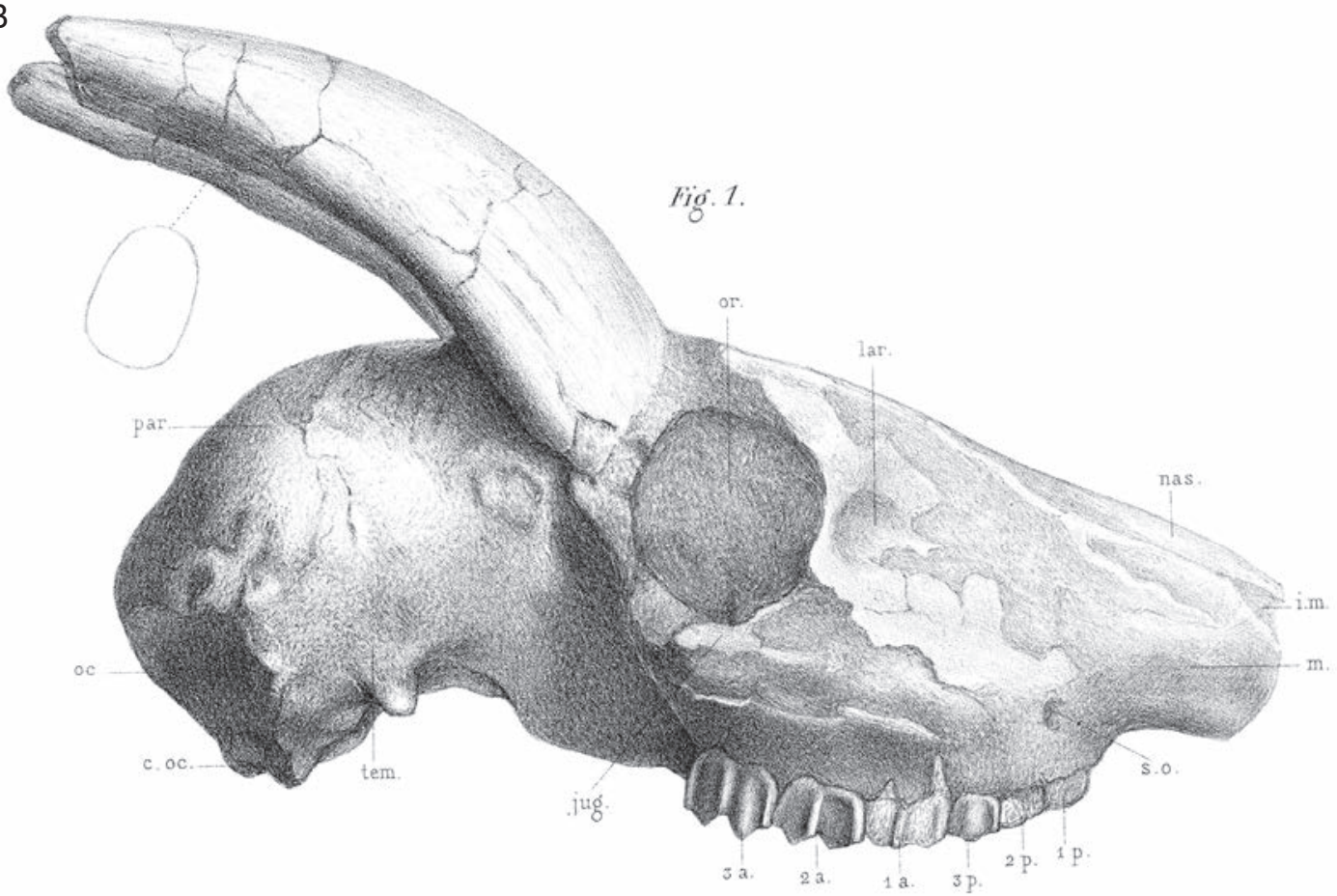

FIG. 10. - Gazella deperdita Gervais in Gervais \& Serres, 1847, crâne MNHN.F.LUB681 Luberon (Miocène supérieur) : A, vue latérale gauche ; B idem, reproduit de Gaudry (1873: pl. 11, fig. 1). Cliché L. Cazes (MNHN). Échelle : $5 \mathrm{~cm}$. 

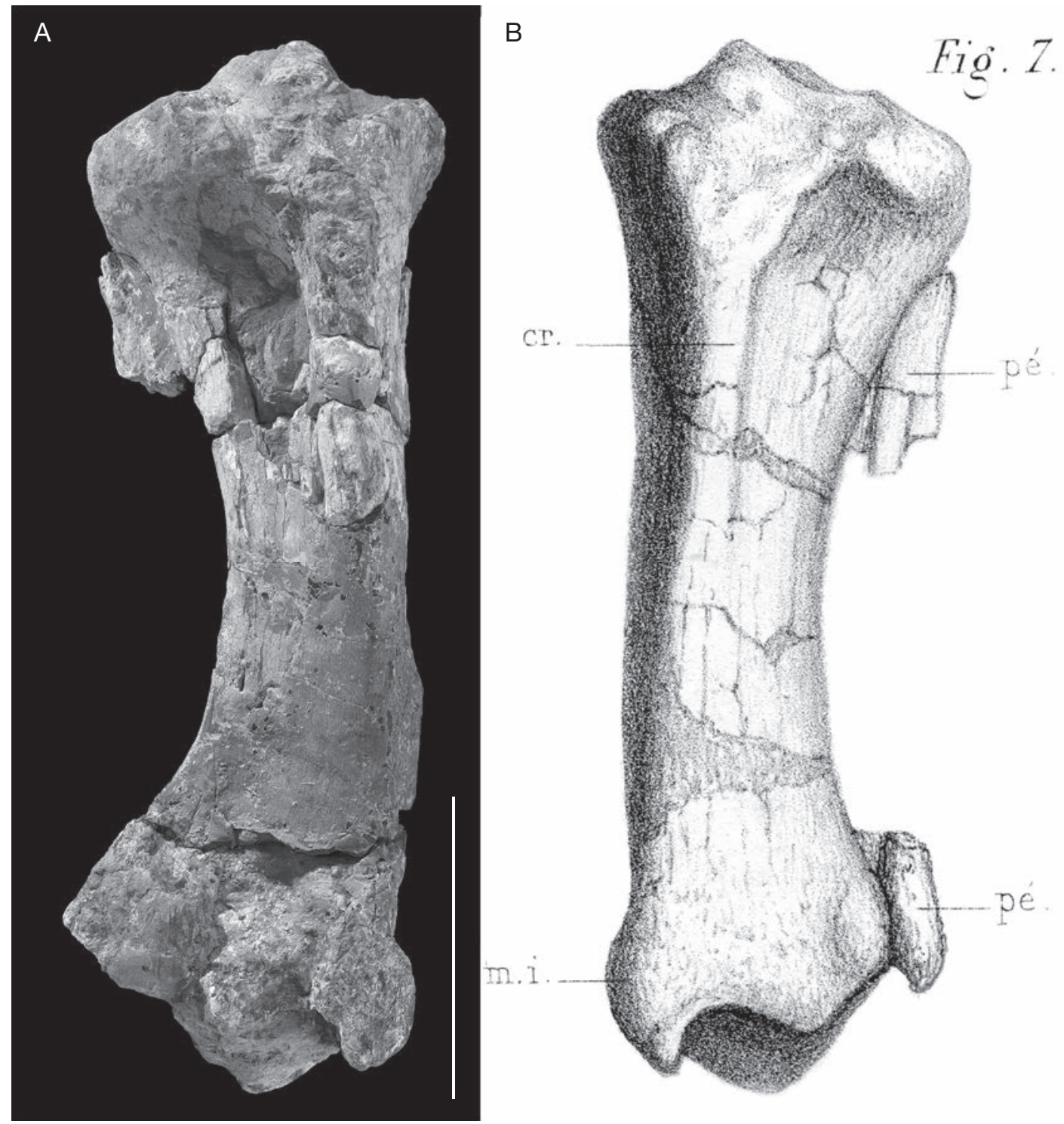

FIG. 11. - Dihoplus schleiermacheri (Kaup, 1832), tibia et fibula droits (MNHN.F.LUB955), Luberon (Miocène supérieur) : A, vue antérieure ; B, idem, reproduit de Gaudry (1873: pl. 4, fig. 7). La fibula a été endommagée depuis la figuration du spécimen par Gaudry. Cliché L. Cazes (MNHN). Échelle : $10 \mathrm{~cm}$.

L'ensemble des données associées pour chacun des spécimens a été entré dans un tableur : chaque spécimen correspond à une ligne du tableur et chaque information est portée dans une colonne. Cette étape s'est avérée ici nécessaire en raison de l'absence de catalogue papier : on ne pouvait prévoir les inter- valles de numéros occupés, les trous, les manques et, surtout, le dernier numéro attribué avant ce travail. Le fichier final produit a ensuite été intégré à la base de données du Muséum, en consultation libre en ligne à l'adresse : http://science.mnhn.fr/ institution/mnhn/collection/f/item/search/form/ 


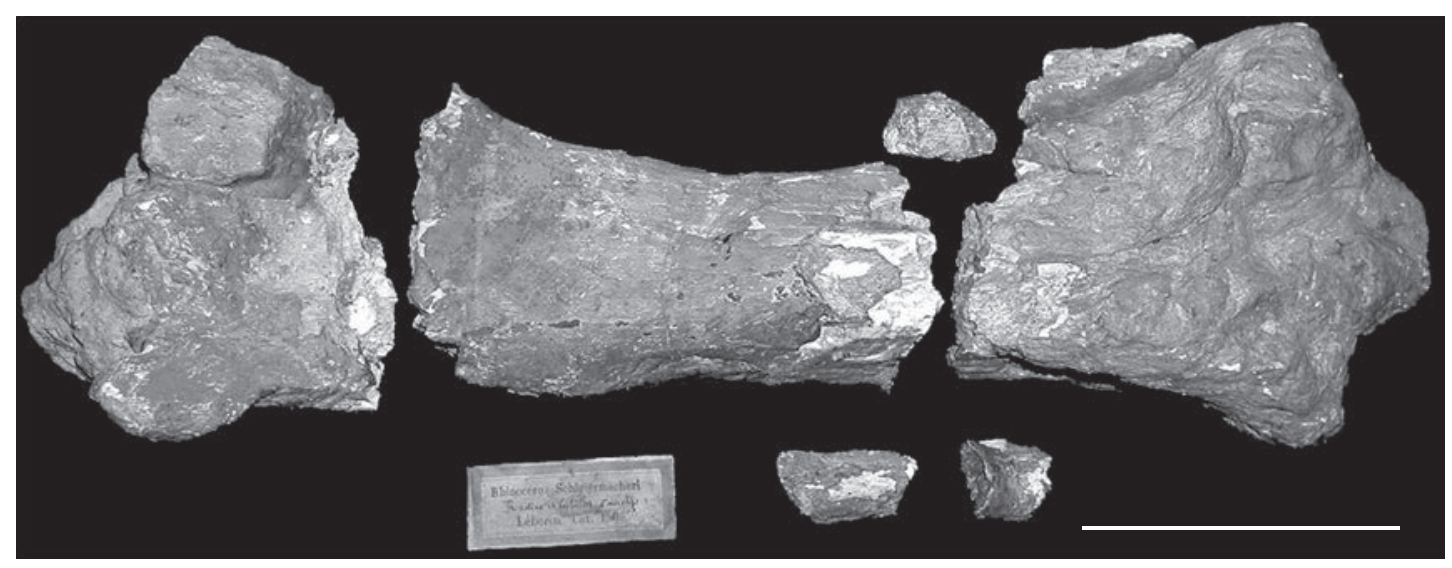

FIG. 12. - Dihoplus schleiermacheri (Kaup, 1832), tibia et fibula droits (MNHN.F.LUB955), Luberon (Miocène supérieur) : vue postérieure du spécimen tel qu'il se présentait avant restauration (voir Fig. 11). Cliché V. Pernègre. Échelle : $10 \mathrm{~cm}$.

\section{La collection aujourd'hui}

Aujourd'hui, la collection de vertébrés dite « du Mont Luberon ", ou " de Cucuron " portant l'acronyme LUB se compose principalement de spécimens recueillis lors des fouilles Gaudry (correspondant aux numéros d'entrée cat. 64 et 1863-1; cat. 156 et 1866-16).

Elle contient aussi, par ordre d'importance du nombre de spécimens, des fossiles provenant des fouilles Christol (Fig. 13) (spécimens numérotés dans l'ancien inventaire paléontologique d'Anatomie Comparée), de l'abbé Caire (idem), de l'abbé Conil (idem) et de Lartet (sans numéro) qui n’a donc pas seulement donné à Gaudry des planches inédites de Bravard mais aussi des fossiles. Â quoi s'ajoutent des spécimens qui ont été donnés au Muséum simultanément au matériel de Gaudry par Gaston de Saporta (1866-6) et enfin, après la publication du travail de Gaudry, par Charles Depéret (1890-25).

La collection comporte actuellement 988 numéros attribués au sigle LUB de la collection de Paléontologie du Muséum national d'Histoire naturelle (MNHN.F.LUB). Cela correspond à 1201 éléments individualisés (voir le catalogue résumé en Annexe 1).

Quatre numéros déjà portés par des fossiles sont invalides, les spécimens provenant en réalité d'autres gisements (voir annexe 2).

Le contrôle des spécimens d'autres gisements européens (tous d'âge Miocène supérieur) représentés dans la collection de paléontologie a été effectué afin de rechercher les spécimens manquants. Ainsi, sept spécimens auparavant mal catalogués ont pu être retrouvés et reportés correctement. Il reste onze spécimens, toujours manquants, qui n'ont pas pu être mis au jour lors de ces recherches. Parmi eux cinq figurés par Gaudry (1873) et Heintz (1971) ne portent vraisemblablement pas de numéros dans le sigle LUB (voir Annexe 2). Les six autres, numérotés LUB, n’ont pas été retrouvés. Ils se situent tous dans l'ancien intervalle réservé à la numérotation des équidés (" Hipparions ") (voir Annexe 2). Enfin, pour être exhaustif, il convient de préciser que quelques spécimens ne font pas partie de la collection de paléontologie. Ils sont conservés au sein de la collection de géologie du Muséum (collection Christol).

\section{CONCLUSION}

En France, la paléontologie évolutive est née, dans la douleur, de l'étude des vertébrés de Pikermi et du Luberon. Cette affirmation n'est pas exagérée. Pour s'en convaincre, évoquons ce commentaire de Dollfus (1909: 276) « On conçoit que les doctrines transformistes de Gaudry, si opposées à celles qui régnaient alors dans l'histoire naturelle et la géologie, n'avaient pas été sans attirer à leur propagateur des difficultés de toutes natures. Nous ne pouvons 


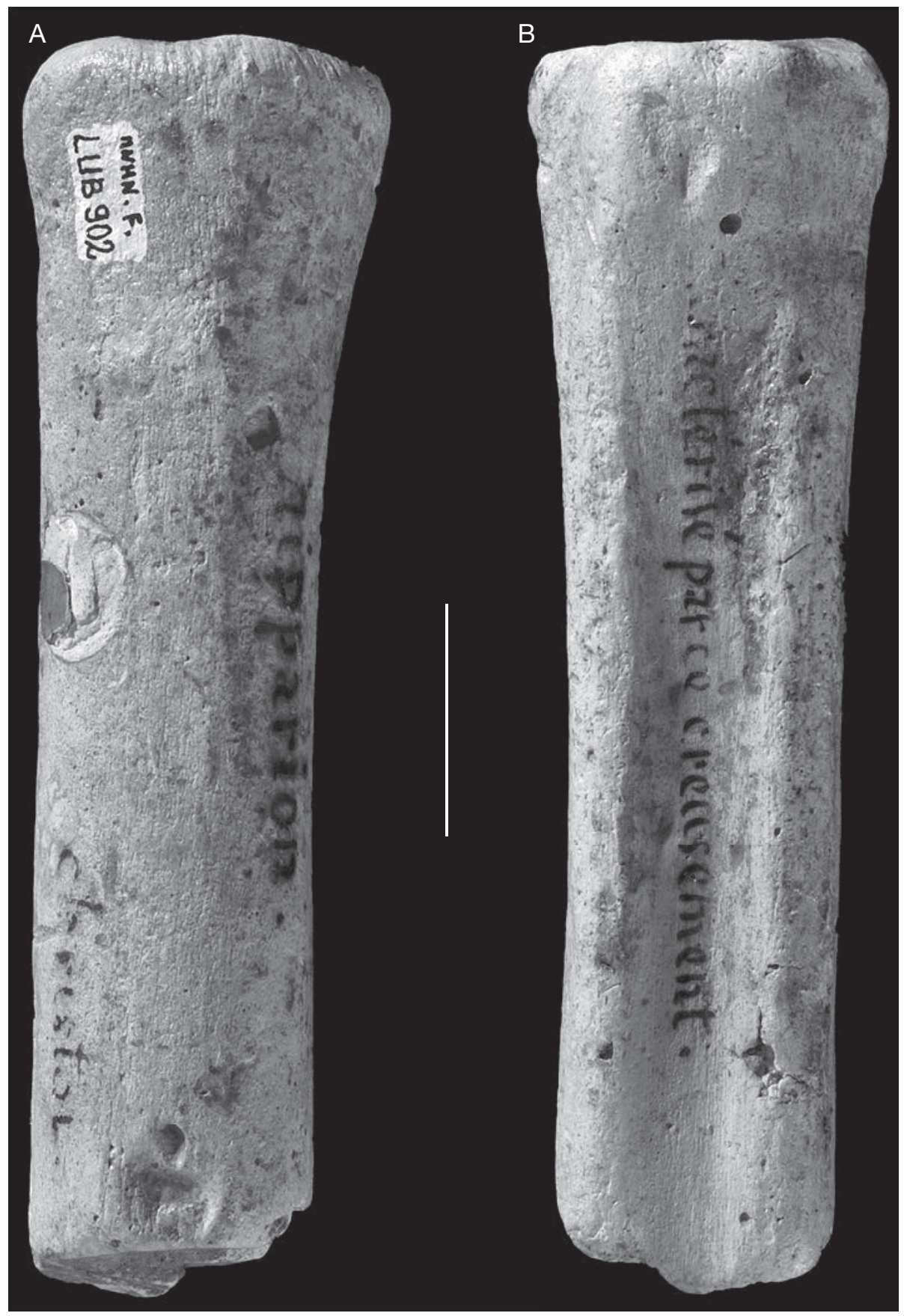

FIG. 13. - Hipparion prostylum Gervais, 1849, moulage de troisième métacarpien droit MNHN.F.LUB902 (collection Christol) : A, vue antérieure ; B, vue postérieure. II est possible que les mentions "Christol », " Hipparion " et « la caractéristique est le creusement » portées par le spécimen aient été écrites par Gaudry lui-même. Clichés L. Cazes (MNHN). Échelle : $2 \mathrm{~cm}$. 
guère nous faire une idée aujourd'hui de la lutte ardente que le maître eût à soutenir de tous les côtés. Le laboratoire de paléontologie était brutalement attaqué de toutes parts : le conseil du Muséum, l'Institut, la Sorbonne, la presse s'en occupaient, la chaire même de paléontologie fut en péril, il s'en fallut de peu qu'elle ne fut disloquée à travers les services des animaux vivants. Gaudry n'avait alors pour le soutenir qu'un petit nombre d'amis".

À l'instar des grandes collections de mammifères fossiles du Miocène supérieur, celles de Pikermi et de Salonique en Grèce, de Maragha en Iran, dont le Muséum national d'Histoire naturelle possède des collections de référence (respectivement Gaudry [1862-1867], Arambourg \& Piveteau [1929] et Mecquenem [1908-1911, 1924-1925]), celle du Luberon est désormais totalement accessible.

Cette mise à niveau revient à faire un éloge de la lenteur, vertu peu appréciée par les temps qui courent. D'une certaine manière, il a fallu 140 ans pour arriver à ce résultat mais surtout, plus sérieusement, 700 heures de travail de conservation conséquent, un bilan horaire qui risque de ne pas être considéré comme rentable au regard du nombre de spécimens traités, critère en passe de devenir le seul qui vaille d'être cité. Aujourd'hui que le projet E-ReColNat vise à numériser les collections d'histoire naturelle en France, le travail présenté ici fait figure de provocation muséale : c'est une goutte d'eau dans une piscine olympique. Cependant, dans l'état où elle est aujourd'hui, la collection des vertébrés fossiles du Luberon est restaurée pour les siècles à venir. Cette perspective historique qui fait fi des modes et des enthousiasmes parfois aussi intenses que passagers est une autre façon de s'inscrire dans la permanence et l'actualité du Muséum national d'Histoire naturelle. Nous ne doutons pas que les fossiles du Luberon reçoivent désormais un nombre encore accru de visiteurs (et, pour cela, une seule adresse internet : http://colhelper.mnhn.fr/). Plus généralement, comme la paléontologie est née en grande partie au Muséum national d'Histoire naturelle, et qu'elle y a connu toutes ses mutations, la collection des fossiles du Muséum, dans son ensemble, présente un double intérêt scientifique et patrimonial sans équivalent. Les archives de la planète sont là, on peut s'y pencher à loisir, aujourd'hui plus que jamais.

\section{Remerciements}

La collection du Luberon a fait l'objet d'un premier traitement (inventaire, restauration) par Manuel Martinez Caceres (vacations du département Histoire de la Terre du MNHN). Lilian Cazes (MNHN) a réalisé la majorité des photographies. France de Lapparent de Broin a expertisé la synonymie des Chelonia. Merci également à Hervé Lelièvre (MNHN) grâce à qui le spécimen MNHN.F.LUB987 est exposé dans la Galerie de Paléontologie du Muséum national d'Histoire naturelle et à Claire Sagne (MNHN) pour sa bienveillante attention tout au long de la remise à niveau de la collection des mammifères fossiles du Miocène supérieur. Nous avons bénéficié des commentaires pertinents et des suggestions astucieuses de Francis Duranthon (MHNT) et d'un rapporteur anonyme.

\section{RÉFÉRENCES}

Arambourg C. \& Piveteau J. 1929. — Les vertébrés du Pontien de Salonique. Annales de Paléontologie 18: 59-138.

Bernor R. L., WoOdburne M. O. \& VAn Couvering J. A. 1980. - A contribution to the chronology of some old World Miocene faunas based on Hipparionine horses. Geobios 13: 705-739.

Bernor R. L., SOlOUnias N., SWisher III C. C. \& VAN Couvering J. A. 1996. - The correlation of three classical "Pikermian" mammal faunas - Maragheh, Samos, and Pikermi - with the European MN Unit System, in Bernor R. L., Fahlbusch V. \& MitTMANN H-W. (éds), The Evolution of Western Eurasian Neogene mammal faunas, Columbia University Press, New York : 137-154.

Bonis L. DE \& Bouvrain G. 1996. - Suidae du Miocène supérieur de Grèce. Bulletin du Muséum national d'Histoire naturelle, Paris, 4ème série, C-18: 107-132.

Bouvrain G. 1977. — Présence de Gazella deperdita (Gervais) 1847 du gisement du Miocène supérieur (Turolien) des Mistrals (Vaucluse, Sud-Est de la France). Geobios 10: 743-747.

Christol J. DE 1832. - [M. Jules de Christol a découvert, dans le département de Vaucluse...] Annales des Sciences et de l'Industrie du Midi de la France 1: $180,181$.

CONRY Y. 1987. - Darwin en perspective. Vrin, Paris, $187 \mathrm{p}$.

DARWIN C. 1888. - C. Darwin to J. L. A. de Quatrefages, in DARWIN F. (éd.), The Life and Letters of Charles Darwin Including an Autobiographical Chapter. Volume III. J. Murray, Londres: 117-118. 
DE BEER G. 1954. - Archaeopteryx lithographica. Trustees of the British Museum, London, 68 p.

DeChaseaux C. 1961. - Moulages endocrâniens de Bovidés fossiles. Annales de Paléontologie 47: 49-73.

DollFus G. 1909. — Albert Gaudry, 1827-1908. Journal de Conchyliologie 57: 274-278.

DURAND J. 1975. — L'idée d'évolution dans l'euvre d'Albert Gaudry (1827-1908). Thèse de Docteur de l'Université de Paris (Mention Sciences) à l'Université de Paris VI-Pierre et Marie Curie. Inédit, 268 p.

FISCHER P. \& TOURNOUËR R. 1873. — Invertébrés fossiles du Mont Léberon, in GAUdry A., FisCHER P. \& TOURnOUËr R. (éds), Animaux fossiles du Mont Léberon (Vaucluse). F. Savy, Paris: 113-175.

GAUDRY A. 1866. - Considérations générales sur les animaux fossiles de Pikermi. F. Savy, Paris, 68 p.

GAUDRY A. 1862-1867. - Animaux fossiles et géologie de l'Attique. F. Savy, Paris, 476 p.

GAUDRY A. 1869. - La théorie de l'évolution et la détermination des terrains; les migrations animales aux époques géologiques. Revue des Cours scientifiques 7: 44-47.

GAUDRY A. 1873. - Vertébrés fossiles du Mont Léberon, in GAudry A., Fischer P. \& TOURNOUËr R. (éds), Animaux fossiles du Mont Léberon (Vaucluse). F. Savy, Paris: 5-112.

GAUDRY A. 1878. - Les enchaînements du monde animal dans les temps géologiques. Mammiferes tertiaires. Masson, Paris, 293 p.

GAUDRY A. 1883. - Les enchaînements du monde animal dans les temps géologiques. Fossiles primaires. Masson, Paris, $320 \mathrm{p}$.

GAUDRY A. 1888. — Les ancêtres de nos animaux dans les temps géologiques. J.-B. Bailllère et Fils, Paris, xv + 296 p.

GAUDRY A. 1890. - Les enchaînements du monde animal dans les temps géologiques. Fosssiles secondaires. Masson, Paris, $322 \mathrm{p}$.

GAUdry A., Fischer P. \& TOURNOUËr R. 1873. - Animaux fossiles du Mont Léberon (Vaucluse). F. Savy, Paris, $180 \mathrm{p}$.
HeINTZ E. 1969. - Le dimorphisme sexuel des appendices frontaux chez Gazella deperdita, Gervais (Bovidae, Artiodactyla, Mammalia) et sa signification phylogénique. Mammalia 33: 626-629.

HEINTZ E. 1971. — Gazella deperdita (Gervais) 1847 (Bovidae, Artiodactyla, mammalia) du Pontien du Mont Lubéron, Vaucluse, France. Annales de Paléontologie 57: 209-229.

HenNIG W. 1965. - Phylogenetic systematics. Annual Review of Entomology 10: 97-116.

Howell F. C. \& PetTer G. 1985. — Étude comparative de certains hyénidés du Miocène moyen et supérieur. Genres : Percrocuta Kretzoi, Allohyaena Kretzoi, Adcrocuta Kretzoi (Mammalia, Carnivora, Hyaenidae). Geobios 18: 419-476.

MacFadden B. J. 1980. - The Miocene horse Hipparion from North America and from the type locality in southern France. Palaeontology 23: 617-636.

MeCQUeneM R. DE 1908-1911. - Contribution à l'étude du gisement des vertébrés de Maragha et de ses environs. Annales d'Histoire naturelle de la Délégation en Perse 1: 27-98.

MECQUENEM R. DE 1924-1925. - Contribution à l'étude des fossiles de Maragha. Annales de Paléontologie 13: 135-160; 14: 29-64.

Skinner M. F. \& MacFadden B. J. 1977. - Cormohipparion n. gen. (Mammalia, Equidae) from the North American Miocene (Barstovian-Clarendonian). Journal of Paleontology 51: 912-926.

SONDAAR P. Y. 1974. - The Hipparion of the Rhone Valley. Geobios 7 : 289-306.

TAssy P. 2006. - Albert Gaudry et l'émergence de la paléontologie darwinienne au XIXe siècle. Annales de Paléontologie 92: 41-70.

TASSY P. 2011. - Trees before and after Darwin. Journal of Zoological Systematics and Evolutionary Research 49: 89-101.

Woodburne M. O. \& Bernor R. L. 1980. - On superspecific groups of some old World Hipparionine horses. Journal of Paleontology 54: 1319-1348.

Soumis le 12 juillet 2013; accepté le 16 janvier 2014; publié le 26 décembre 2014. 
ANNEXE 1. - Catalogue simplifié des vertébrés miocènes du Mont Luberon (Vaucluse, France). Les spécimens sont consultables sur le site http://coldb.mnhn.fr.

\begin{tabular}{|c|c|c|c|}
\hline Numéro de spécimen & Anatomie & Statut nomenclatural & Espèce \\
\hline MNHN.F.LUB1 & mandibule & & Hipparion prostylum \\
\hline MNHN.F.LUB2 & mandibule & & Hipparion prostylum \\
\hline MNHN.F.LUB3 & crâne & & Hipparion prostylum \\
\hline MNHN.F.LUB4 & mandibule & & Hipparion prostylum \\
\hline MNHN.F.LUB5 & mandibule & FIGURÉ & Hipparion prostylum \\
\hline MNHN.F.LUB6a & dentition supérieure & & Hipparion prostylum \\
\hline MNHN.F.LUB6b & dentition supérieure & FIGURÉ & Hipparion prostylum \\
\hline MNHN.F.LUB6c & dentition supérieure & & Hipparion prostylum \\
\hline MNHN.F.LUB6d & dentition supérieure & & Hipparion prostylum \\
\hline MNHN.F.LUB6e & crâne & & Hipparion prostylum \\
\hline MNHN.F.LUB7 & dentition inférieure & FIGURÉ & Hipparion prostylum \\
\hline MNHN.F.LUB8 & dentition supérieure & & Hipparion prostylum \\
\hline MNHN.F.LUB9 & ceinture & & Incertae sedis \\
\hline MNHN.F.LUB10 & dentition supérieure & FIGURÉ & Hipparion prostylum \\
\hline MNHN.F.LUB11 & dentition supérieure & FIGURÉ & Hipparion prostylum \\
\hline MNHN.F.LUB12 & mandibule & & Hipparion prostylum \\
\hline MNHN.F.LUB13 & dentition supérieure & & Hipparion prostylum \\
\hline MNHN.F.LUB14 & mandibule & FIGURÉ & Hipparion prostylum \\
\hline MNHN.F.LUB15 & crâne & & Hipparion prostylum \\
\hline MNHN.F.LUB16 & dentition supérieure & & Hipparion prostylum \\
\hline MNHN.F.LUB17 & dentition supérieure & FIGURÉ & Hipparion prostylum \\
\hline MNHN.F.LUB18 & crâne & FIGURÉ & Hipparion prostylum \\
\hline MNHN.F.LUB19 & mandibule & & Hipparion prostylum \\
\hline MNHN.F.LUB20 & mandibule & & Hipparion prostylum \\
\hline MNHN.F.LUB21 & mandibule & & Hipparion prostylum \\
\hline MNHN.F.LUB22 & dentition inférieure & & Hipparion prostylum \\
\hline MNHN.F.LUB23 & dentition supérieure & & Hipparion prostylum \\
\hline MNHN.F.LUB24 & sésamoïde & & Hipparion prostylum \\
\hline MNHN.F.LUB25 & sésamoïde & & Hipparion prostylum \\
\hline MNHN.F.LUB26 & mandibule & FIGURÉ & Hipparion prostylum \\
\hline MNHN.F.LUB27 & mandibule & & Hipparion prostylum \\
\hline MNHN.F.LUB28 & mandibule & & Hipparion prostylum \\
\hline MNHN.F.LUB29 & mandibule & & Hipparion prostylum \\
\hline MNHN.F.LUB30 & mandibule & & Hipparion prostylum \\
\hline MNHN.F.LUB31 & dentition supérieure & & Hipparion prostylum \\
\hline MNHN.F.LUB32 & mandibule & & Hipparion prostylum \\
\hline MNHN.F.LUB33 & crâne & & Hipparion prostylum \\
\hline MNHN.F.LUB34 & dentition supérieure & & Hipparion prostylum \\
\hline MNHN.F.LUB35 & mandibule & & Hipparion prostylum \\
\hline MNHN.F.LUB36 & dentition inférieure & & Hipparion prostylum \\
\hline MNHN.F.LUB37 & dentition inférieure & & Hipparion prostylum \\
\hline MNHN.F.LUB38 & dentition supérieure & & Hipparion prostylum \\
\hline MNHN.F.LUB39 & métapode & & Hipparion prostylum \\
\hline MNHN.F.LUB40 & mandibule & FIGURÉ & Hipparion prostylum \\
\hline MNHN.F.LUB41a & dentition supérieure & & Hipparion prostylum \\
\hline MNHN.F.LUB41b & dentition supérieure & & Hipparion prostylum \\
\hline MNHN.F.LUB41c & dentition supérieure & & Hipparion prostylum \\
\hline MNHN.F.LUB42 & dentition inférieure & & Hipparion prostylum \\
\hline MNHN.F.LUB43 & crâne & & Hipparion prostylum \\
\hline MNHN.F.LUB44 & crâne & & Hipparion prostylum \\
\hline MNHN.F.LUB45 & crâne & & Hipparion prostylum \\
\hline MNHN.F.LUB46 & métapode & & Hipparion prostylum \\
\hline MNHN.F.LUB47 & métapode & & Hipparion prostylum \\
\hline MNHN.F.LUB48 & métapode & & Hipparion prostylum \\
\hline MNHN.F.LUB49 & métapode & & Hipparion prostylum \\
\hline
\end{tabular}


ANNEXE 1. - Suite.

\begin{tabular}{|c|c|c|}
\hline Numéro de spécimen & Statut nomenclatural & Espèce \\
\hline MNHN.F.LUB50 & métapode & Hipparion prostylum \\
\hline MNHN.F.LUB51 & mandibule & Hipparion prostylum \\
\hline MNHN.F.LUB52 & mandibule & Hipparion prostylum \\
\hline MNHN.F.LUB53 & métapode & Hipparion prostylum \\
\hline LUB 54 & spécimen non retrouvé en collection & \\
\hline MNHN.F.LUB55 & métapode & Hipparion prostylum \\
\hline MNHN.F.LUB56 & métapode & Hipparion prostylum \\
\hline MNHN.F.LUB57 & métapode & Hipparion prostylum \\
\hline MNHN.F.LUB58 & métapode & Hipparion prostylum \\
\hline MNHN.F.LUB59 & vertèbre & Hipparion prostylum \\
\hline MNHN.F.LUB60 & vertèbre & Hipparion prostylum \\
\hline MNHN.F.LUB61 & mandibule & Hipparion prostylum \\
\hline MNHN.F.LUB62 & vertèbre & Hipparion prostylum \\
\hline MNHN.F.LUB63a & vertèbre & Hipparion prostylum \\
\hline MNHN.F.LUB63b & vertèbre & Hipparion prostylum \\
\hline MNHN.F.LUB64 & vertèbre & Incertae sedis \\
\hline MNHN.F.LUB65a & vertèbre & Tragoportax amalthea \\
\hline MNHN.F.LUB65b & vertèbre & Tragoportax amalthea \\
\hline MNHN.F.LUB66 & vertèbre & Tragoportax amalthea \\
\hline MNHN.F.LUB67 & vertèbre & Tragoportax amalthea \\
\hline MNHN.F.LUB68 & vertèbre & Hipparion prostylum \\
\hline MNHN.F.LUB69 & vertèbre & Hipparion prostylum \\
\hline MNHN.F.LUB70 & vertèbre & Hipparion prostylum \\
\hline MNHN.F.LUB71 & vertèbre & Hipparion prostylum \\
\hline MNHN.F.LUB72 & vertèbre & Hipparion prostylum \\
\hline MNHN.F.LUB73 & vertèbre & Incertae sedis \\
\hline MNHN.F.LUB74 & vertèbre & Hipparion prostylum \\
\hline MNHN.F.LUB75 & vertèbre & Hipparion prostylum \\
\hline MNHN.F.LUB76 & basipode & Hipparion prostylum \\
\hline MNHN.F.LUB77 & basipode & Hipparion prostylum \\
\hline MNHN.F.LUB78 & basipode & Hipparion prostylum \\
\hline MNHN.F.LUB79 & basipode & Hipparion prostylum \\
\hline MNHN.F.LUB80 & basipode & Hipparion prostylum \\
\hline MNHN.F.LUB81 & vertèbre & Hipparion prostylum \\
\hline MNHN.F.LUB82 & vertèbre & Incertae sedis \\
\hline MNHN.F.LUB83 & vertèbre & Incertae sedis \\
\hline MNHN.F.LUB84 & vertèbre & Hipparion prostylum \\
\hline MNHN.F.LUB85 & vertèbre & Hipparion prostylum \\
\hline MNHN.F.LUB86 & basipode & Hipparion prostylum \\
\hline MNHN.F.LUB87 & vertèbre & Hipparion prostylum \\
\hline MNHN.F.LUB88 & crâne & Hipparion prostylum \\
\hline MNHN.F.LUB89 & mandibule & Hipparion prostylum \\
\hline MNHN.F.LUB90 & dentition inférieure & Hipparion prostylum \\
\hline MNHN.F.LUB91 & crâne & Hipparion prostylum \\
\hline MNHN.F.LUB92 & ceinture & Hipparion prostylum \\
\hline MNHN.F.LUB93 & ceinture & Hipparion prostylum \\
\hline MNHN.F.LUB94 & FIGURÉ & Hipparion prostylum \\
\hline MNHN.F.LUB95 & crâne & Hipparion prostylum \\
\hline MNHN.F.LUB96 & mandibule & Hipparion prostylum \\
\hline MNHN.F.LUB97 & crâne & Hipparion prostylum \\
\hline MNHN.F.LUB98 & mandibule & Hipparion prostylum \\
\hline MNHN.F.LUB99 & mandibule & Hipparion prostylum \\
\hline MNHN.F.LUB100 & mandibule & Hipparion prostylum \\
\hline MNHN.F.LUB101 & mandibule & Hipparion prostylum \\
\hline MNHN.F.LUB102 & mandibule & Hipparion prostylum \\
\hline MNHN.F.LUB103 & stylopode & Hipparion prostylum \\
\hline
\end{tabular}


ANNEXE 1. - Suite.

\begin{tabular}{|c|c|c|}
\hline Numéro de spécimen & Statut nomenclatural & Espèce \\
\hline MNHN.F.LUB104 & zeugopode & Helladotherium duvernoyi \\
\hline MNHN.F.LUB105 & mandibule & Hipparion prostylum \\
\hline MNHN.F.LUB106 & ceinture & Incertae sedis \\
\hline MNHN.F.LUB107 & vertèbre & Hipparion prostylum \\
\hline MNHN.F.LUB108 & vertèbre & Hipparion prostylum \\
\hline MNHN.F.LUB109a & vertèbre & Hipparion prostylum \\
\hline MNHN.F.LUB109b & vertèbre & Hipparion prostylum \\
\hline MNHN.F.LUB109c & vertèbre & Hipparion prostylum \\
\hline MNHN.F.LUB109d & vertèbre & Hipparion prostylum \\
\hline MNHN.F.LUB110 & vertèbre & Hipparion prostylum \\
\hline MNHN.F.LUB111 & vertèbre & Hipparion prostylum \\
\hline MNHN.F.LUB112 & vertèbre & Incertae sedis \\
\hline MNHN.F.LUB113 & vertèbre & Incertae sedis \\
\hline MNHN.F.LUB114 & vertèbre & Hipparion prostylum \\
\hline MNHN.F.LUB115 & vertèbre & Hipparion prostylum \\
\hline MNHN.F.LUB116 & vertèbre & Hipparion prostylum \\
\hline MNHN.F.LUB117 & vertèbre & Hipparion prostylum \\
\hline MNHN.F.LUB118 & vertèbre & Tragoportax amalthea \\
\hline MNHN.F.LUB119 & vertèbre & Hipparion prostylum \\
\hline MNHN.F.LUB120 & vertèbre & Hipparion prostylum \\
\hline MNHN.F.LUB121 & basipode & Hipparion prostylum \\
\hline MNHN.F.LUB122 & basipode & Hipparion prostylum \\
\hline MNHN.F.LUB123 & dentition inférieure & Hipparion prostylum \\
\hline MNHN.F.LUB124 & dentition supérieure & Hipparion prostylum \\
\hline MNHN.F.LUB125 & dentition inférieure & Hipparion prostylum \\
\hline MNHN.F.LUB126 & dentition inférieure & Hipparion prostylum \\
\hline MNHN.F.LUB127 & vertèbre & Tragoportax amalthea \\
\hline MNHN.F.LUB128 & vertèbre & Tragoportax amalthea \\
\hline MNHN.F.LUB129 & dentition inférieure & Hipparion prostylum \\
\hline LUB 130 & $\mathrm{n}^{\circ}$ invalide. Spécimen non Luberon & \\
\hline MNHN.F.LUB131 & dentition supérieure & Hipparion prostylum \\
\hline MNHN.F.LUB132 & dentition inférieure & Hipparion prostylum \\
\hline MNHN.F.LUB133 & dentition supérieure & Hipparion prostylum \\
\hline LUB 134 & $\mathrm{n}^{\circ}$ invalide. Spécimen non Luberon & \\
\hline MNHN.F.LUB135 & dentition inférieure & Hipparion prostylum \\
\hline MNHN.F.LUB136 & dentition inférieure & Hipparion prostylum \\
\hline MNHN.F.LUB137 & dentition inférieure & Hipparion prostylum \\
\hline LUB 138 & spécimen non retrouvé en collection & \\
\hline MNHN.F.LUB139 & dentition inférieure $\quad$ FIGURÉ & Hipparion prostylum \\
\hline MNHN.F.LUB140 & vertèbre & Hipparion prostylum \\
\hline MNHN.F.LUB141 & vertèbre & Hipparion prostylum \\
\hline MNHN.F.LUB142 & vertèbre & Hipparion prostylum \\
\hline MNHN.F.LUB143 & basipode & Hipparion prostylum \\
\hline MNHN.F.LUB144 & vertèbre & Hipparion prostylum \\
\hline MNHN.F.LUB145 & vertèbre & Hipparion prostylum \\
\hline MNHN.F.LUB146 & vertèbre & Hipparion prostylum \\
\hline MNHN.F.LUB147 & vertèbre & Hipparion prostylum \\
\hline MNHN.F.LUB148 & phalange & Hipparion prostylum \\
\hline MNHN.F.LUB149 & phalange & Hipparion prostylum \\
\hline MNHN.F.LUB150 & phalange & Hipparion prostylum \\
\hline MNHN.F.LUB151 & phalange & Hipparion prostylum \\
\hline MNHN.F.LUB152 & basipode & Hipparion prostylum \\
\hline MNHN.F.LUB153 & métapode & Hipparion prostylum \\
\hline MNHN.F.LUB154 & basipode & Hipparion prostylum \\
\hline MNHN.F.LUB155 & basipode & Hipparion prostylum \\
\hline MNHN.F.LUB156 & zeugopode & Hipparion prostylum \\
\hline
\end{tabular}


ANNEXE 1. - Suite.

\begin{tabular}{|c|c|c|}
\hline Numéro de spécimen & Statut nomenclatural & Espèce \\
\hline MNHN.F.LUB157 & basipode & Hipparion prostylum \\
\hline MNHN.F.LUB158 & basipode & Hipparion prostylum \\
\hline MNHN.F.LUB159 & basipode & Hipparion prostylum \\
\hline MNHN.F.LUB160 & basipode & Hipparion prostylum \\
\hline MNHN.F.LUB161 & basipode & Hipparion prostylum \\
\hline MNHN.F.LUB162 & basipode & Hipparion prostylum \\
\hline MNHN.F.LUB163 & basipode & Hipparion prostylum \\
\hline MNHN.F.LUB164 & basipode & Hipparion prostylum \\
\hline MNHN.F.LUB165 & basipode & Hipparion prostylum \\
\hline MNHN.F.LUB166 & basipode & Hipparion prostylum \\
\hline MNHN.F.LUB167 & basipode & Hipparion prostylum \\
\hline MNHN.F.LUB168 & basipode & Hipparion prostylum \\
\hline MNHN.F.LUB169 & basipode & Hipparion prostylum \\
\hline MNHN.F.LUB170 & basipode & Hipparion prostylum \\
\hline MNHN.F.LUB171 & basipode & Hipparion prostylum \\
\hline MNHN.F.LUB172 & basipode & Hipparion prostylum \\
\hline MNHN.F.LUB173 & vertèbre & Hipparion prostylum \\
\hline MNHN.F.LUB174 & os indet. & Incertae sedis \\
\hline MNHN.F.LUB175 & vertèbre & Hipparion prostylum \\
\hline MNHN.F.LUB176 & vertèbre & Incertae sedis \\
\hline MNHN.F.LUB177 & zeugopode & Incertae sedis \\
\hline MNHN.F.LUB178 & stylopode & Hipparion prostylum \\
\hline MNHN.F.LUB179 & stylopode & Hipparion prostylum \\
\hline MNHN.F.LUB180 & basipode & Hipparion prostylum \\
\hline MNHN.F.LUB181 & zeugopode & Helladotherium duvernoyi \\
\hline LUB 182 & spécimen non retrouvé en collection & \\
\hline MNHN.F.LUB183 & crâne & Hipparion prostylum \\
\hline MNHN.F.LUB184 & dentition supérieure & Hipparion prostylum \\
\hline MNHN.F.LUB185 & basipode & Hipparion prostylum \\
\hline MNHN.F.LUB186 & métapode & Hipparion prostylum \\
\hline LUB 187 & spécimen non retrouvé en collection & \\
\hline MNHN.F.LUB188 & dentition inférieure & Hipparion prostylum \\
\hline MNHN.F.LUB189 & métapode & Hipparion prostylum \\
\hline MNHN.F.LUB190 & mandibule & Hipparion prostylum \\
\hline MNHN.F.LUB191 & dentition inférieure & Hipparion prostylum \\
\hline MNHN.F.LUB192 & dentition supérieure & Hipparion prostylum \\
\hline LUB 193 & spécimen non retrouvé en collection & \\
\hline MNHN.F.LUB194 & dentition supérieure & Hipparion prostylum \\
\hline MNHN.F.LUB195 & dentition supérieure & Hipparion prostylum \\
\hline MNHN.F.LUB196 & dentition supérieure & Hipparion prostylum \\
\hline MNHN.F.LUB197 & dentition supérieure & Hipparion prostylum \\
\hline MNHN.F.LUB198 & dentition supérieure & Hipparion prostylum \\
\hline MNHN.F.LUB199 & dentition supérieure & Hipparion prostylum \\
\hline MNHN.F.LUB200 & crâne & Hipparion prostylum \\
\hline MNHN.F.LUB201 & dentition supérieure & Hipparion prostylum \\
\hline MNHN.F.LUB202 & dentition supérieure & Hipparion prostylum \\
\hline MNHN.F.LUB203 & dentition supérieure & Hipparion prostylum \\
\hline MNHN.F.LUB204 & dentition supérieure & Hipparion prostylum \\
\hline MNHN.F.LUB205 & mandibule & Hipparion prostylum \\
\hline MNHN.F.LUB206 & crâne & Hipparion prostylum \\
\hline MNHN.F.LUB207 & crâne & Hipparion prostylum \\
\hline MNHN.F.LUB208 & crâne & Hipparion prostylum \\
\hline MNHN.F.LUB209a & dentition supérieure & Hipparion prostylum \\
\hline MNHN.F.LUB209b & dentition supérieure & Hipparion prostylum \\
\hline MNHN.F.LUB210 & métapode & Hipparion prostylum \\
\hline MNHN.F.LUB211 & basipode & Hipparion prostylum \\
\hline
\end{tabular}


ANNEXE 1. - Suite.

\begin{tabular}{|c|c|c|}
\hline Numéro de spécimen & Statut nomenclatural & Espèce \\
\hline MNHN.F.LUB212 & phalange & Hipparion prostylum \\
\hline MNHN.F.LUB213 & crâne & Hipparion prostylum \\
\hline MNHN.F.LUB214 & basipode & Hipparion prostylum \\
\hline MNHN.F.LUB215 & mandibule & Hipparion prostylum \\
\hline MNHN.F.LUB216 & basipode & Hipparion prostylum \\
\hline MNHN.F.LUB217 & métapode & Hipparion prostylum \\
\hline MNHN.F.LUB218 & zeugopode & Hipparion prostylum \\
\hline MNHN.F.LUB219 & zeugopode & Hipparion prostylum \\
\hline MNHN.F.LUB220 & métapode & Hipparion prostylum \\
\hline MNHN.F.LUB221 & métapode & Hipparion prostylum \\
\hline MNHN.F.LUB222 & métapode & Hipparion prostylum \\
\hline MNHN.F.LUB223 & métapode & Hipparion prostylum \\
\hline MNHN.F.LUB224 & métapode & Hipparion prostylum \\
\hline MNHN.F.LUB225a & métapode & Hipparion prostylum \\
\hline MNHN.F.LUB225b & métapode & Hipparion prostylum \\
\hline MNHN.F.LUB226 & basipode & Hipparion prostylum \\
\hline MNHN.F.LUB227 & basipode & Hipparion prostylum \\
\hline MNHN.F.LUB228 & basipode & Hipparion prostylum \\
\hline MNHN.F.LUB229 & métapode & Hipparion prostylum \\
\hline MNHN.F.LUB230 & crâne & Hipparion prostylum \\
\hline MNHN.F.LUB231 & métapode & Hipparion prostylum \\
\hline LUB 232 & $\mathrm{n}^{\circ}$ invalide. Spécimen non Luberon & \\
\hline MNHN.F.LUB233 & basipode & Hipparion prostylum \\
\hline MNHN.F.LUB234 & basipode & Hipparion prostylum \\
\hline MNHN.F.LUB235 & stylopode & Hipparion prostylum \\
\hline MNHN.F.LUB236 & zeugopode & Hipparion prostylum \\
\hline MNHN.F.LUB237 & phalange & Hipparion prostylum \\
\hline MNHN.F.LUB238 & métapode & Hipparion prostylum \\
\hline MNHN.F.LUB239 & métapode & Hipparion prostylum \\
\hline MNHN.F.LUB240a & zeugopode & Hipparion prostylum \\
\hline MNHN.F.LUB240b & zeugopode & Hipparion prostylum \\
\hline MNHN.F.LUB241 & zeugopode & Hipparion prostylum \\
\hline MNHN.F.LUB242 & dentition supérieure & Hipparion prostylum \\
\hline MNHN.F.LUB243 & dentition supérieure & Hipparion prostylum \\
\hline MNHN.F.LUB244 & dentition supérieure & Hipparion prostylum \\
\hline LUB 245 & $\mathrm{n}^{\circ}$ invalide. Spécimen non Luberon & \\
\hline MNHN.F.LUB246 & dentition supérieure & Hipparion prostylum \\
\hline MNHN.F.LUB247 & dentition supérieure & Hipparion prostylum \\
\hline MNHN.F.LUB248 & dentition supérieure & Hipparion prostylum \\
\hline MNHN.F.LUB249 & dentition supérieure & Hipparion prostylum \\
\hline MNHN.F.LUB250 & dentition supérieure & Hipparion prostylum \\
\hline MNHN.F.LUB251 & dentition supérieure & Hipparion prostylum \\
\hline MNHN.F.LUB252 & dentition supérieure & Hipparion prostylum \\
\hline MNHN.F.LUB253 & dentition inférieure & Hipparion prostylum \\
\hline MNHN.F.LUB254 & dentition inférieure & Hipparion prostylum \\
\hline MNHN.F.LUB255 & dentition inférieure & Hipparion prostylum \\
\hline MNHN.F.LUB256 & mandibule & Hipparion prostylum \\
\hline MNHN.F.LUB257a & basipode & Hipparion prostylum \\
\hline MNHN.F.LUB257b & basipode & Hipparion prostylum \\
\hline MNHN.F.LUB258 & basipode & Hipparion prostylum \\
\hline MNHN.F.LUB259 & basipode & Hipparion prostylum \\
\hline MNHN.F.LUB260 & basipode & Hipparion prostylum \\
\hline MNHN.F.LUB261 & basipode & Hipparion prostylum \\
\hline MNHN.F.LUB262 & basipode & Hipparion prostylum \\
\hline MNHN.F.LUB263 & basipode & Hipparion prostylum \\
\hline MNHN.F.LUB264 & basipode & Hipparion prostylum \\
\hline
\end{tabular}


ANNEXE 1. - Suite.

\begin{tabular}{|c|c|c|c|}
\hline Numéro de spécimen & Anatomie & Statut nomenclatural & Espèce \\
\hline MNHN.F.LUB265 & basipode & & Hipparion prostylum \\
\hline MNHN.F.LUB266 & basipode & & Hipparion prostylum \\
\hline MNHN.F.LUB267 & phalange & & Hipparion prostylum \\
\hline MNHN.F.LUB268 & phalange & & Hipparion prostylum \\
\hline MNHN.F.LUB269 & phalange & & Hipparion prostylum \\
\hline MNHN.F.LUB270 & phalange & & Hipparion prostylum \\
\hline MNHN.F.LUB271 & stylopode & & Hipparion prostylum \\
\hline MNHN.F.LUB272 & stylopode & & Hipparion prostylum \\
\hline MNHN.F.LUB273 & stylopode & & Hipparion prostylum \\
\hline MNHN.F.LUB274 & stylopode & & Hipparion prostylum \\
\hline MNHN.F.LUB275 & stylopode & & Hipparion prostylum \\
\hline MNHN.F.LUB276 & basipode & & Hipparion prostylum \\
\hline MNHN.F.LUB277 & basipode & & Hipparion prostylum \\
\hline MNHN.F.LUB278 & basipode & & Hipparion prostylum \\
\hline MNHN.F.LUB279 & basipode & & Hipparion prostylum \\
\hline MNHN.F.LUB280a & basipode & & Hipparion prostylum \\
\hline MNHN.F.LUB280b & basipode & & Hipparion prostylum \\
\hline MNHN.F.LUB280c & basipode & & Hipparion prostylum \\
\hline MNHN.F.LUB280d & basipode & & Hipparion prostylum \\
\hline MNHN.F.LUB280e & basipode & & Hipparion prostylum \\
\hline MNHN.F.LUB280f & basipode & & Hipparion prostylum \\
\hline MNHN.F.LUB281a & zeugopode & & Hipparion prostylum \\
\hline MNHN.F.LUB281b & zeugopode & & Hipparion prostylum \\
\hline MNHN.F.LUB282 & stylopode & & Hipparion prostylum \\
\hline MNHN.F.LUB283 & stylopode & & Hipparion prostylum \\
\hline MNHN.F.LUB284 & stylopode & & Hipparion prostylum \\
\hline MNHN.F.LUB285 & stylopode & & Hipparion prostylum \\
\hline MNHN.F.LUB286a & zeugopode & & Hipparion prostylum \\
\hline MNHN.F.LUB286b & crâne & & Gazella deperdita \\
\hline MNHN.F.LUB287 & zeugopode & & Hipparion prostylum \\
\hline MNHN.F.LUB288 & zeugopode & & Hipparion prostylum \\
\hline MNHN.F.LUB289a & métapode & & Hipparion prostylum \\
\hline MNHN.F.LUB289b & métapode & & Hipparion prostylum \\
\hline MNHN.F.LUB289c & métapode & & Hipparion prostylum \\
\hline MNHN.F.LUB290 & métapode & & Hipparion prostylum \\
\hline MNHN.F.LUB291 & basipode & & Hipparion prostylum \\
\hline MNHN.F.LUB292 & basipode & & Hipparion prostylum \\
\hline MNHN.F.LUB293 & basipode & & Hipparion prostylum \\
\hline MNHN.F.LUB294 & basipode & & Hipparion prostylum \\
\hline MNHN.F.LUB295 & basipode & & Hipparion prostylum \\
\hline MNHN.F.LUB296 & basipode & & Hipparion prostylum \\
\hline MNHN.F.LUB297a & métapode & & Hipparion prostylum \\
\hline MNHN.F.LUB297b & basipode & & Hipparion prostylum \\
\hline MNHN.F.LUB298 & basipode & & Hipparion prostylum \\
\hline MNHN.F.LUB299 & basipode & & Hipparion prostylum \\
\hline MNHN.F.LUB300 & zeugopode & & Hipparion prostylum \\
\hline MNHN.F.LUB301 & zeugopode & & Hipparion prostylum \\
\hline MNHN.F.LUB302 & zeugopode & & Hipparion prostylum \\
\hline MNHN.F.LUB303 & métapode & FIGURÉ & Hipparion prostylum \\
\hline MNHN.F.LUB304 & métapode & & Hipparion prostylum \\
\hline MNHN.F.LUB305a & métapode & & Hipparion prostylum \\
\hline MNHN.F.LUB305b & métapode & & Hipparion prostylum \\
\hline MNHN.F.LUB306 & métapode & & Hipparion prostylum \\
\hline MNHN.F.LUB307 & métapode & & Hipparion prostylum \\
\hline MNHN.F.LUB308 & métapode & & Hipparion prostylum \\
\hline MNHN.F.LUB309 & métapode & & Hipparion prostylum \\
\hline
\end{tabular}


ANNEXE 1. - Suite.

\begin{tabular}{|c|c|c|c|}
\hline Numéro de spécimen & Anatomie & Statut nomenclatural & Espèce \\
\hline MNHN.F.LUB310 & métapode & & Hipparion prostylum \\
\hline MNHN.F.LUB311 & zeugopode & & Hipparion prostylum \\
\hline MNHN.F.LUB312 & zeugopode & & Hipparion prostylum \\
\hline MNHN.F.LUB313 & zeugopode & & Hipparion prostylum \\
\hline MNHN.F.LUB314 & zeugopode & & Hipparion prostylum \\
\hline MNHN.F.LUB315a & zeugopode & & Hipparion prostylum \\
\hline MNHN.F.LUB315b & zeugopode & & Hipparion prostylum \\
\hline MNHN.F.LUB316 & zeugopode & & Hipparion prostylum \\
\hline MNHN.F.LUB317a & métapode & & Hipparion prostylum \\
\hline MNHN.F.LUB317b & métapode & & Hipparion prostylum \\
\hline MNHN.F.LUB317c & métapode & & Hipparion prostylum \\
\hline MNHN.F.LUB317d & basipode & & Hipparion prostylum \\
\hline MNHN.F.LUB317e & basipode & & Hipparion prostylum \\
\hline MNHN.F.LUB318a & basipode & & Hipparion prostylum \\
\hline MNHN.F.LUB318b & basipode & & Hipparion prostylum \\
\hline MNHN.F.LUB318c & basipode & & Hipparion prostylum \\
\hline MNHN.F.LUB318d & phalange & & Hipparion prostylum \\
\hline MNHN.F.LUB319 & stylopode & & Hipparion prostylum \\
\hline MNHN.F.LUB320 & métapode & & Hipparion prostylum \\
\hline MNHN.F.LUB321a & métapode & & Hipparion prostylum \\
\hline MNHN.F.LUB321b & métapode & & Hipparion prostylum \\
\hline MNHN.F.LUB322a & métapode & & Hipparion prostylum \\
\hline MNHN.F.LUB322b & métapode & & Hipparion prostylum \\
\hline MNHN.F.LUB323 & métapode & & Hipparion prostylum \\
\hline MNHN.F.LUB324 & métapode & & Hipparion prostylum \\
\hline MNHN.F.LUB325 & métapode & & Hipparion prostylum \\
\hline MNHN.F.LUB326a & métapode & & Hipparion prostylum \\
\hline MNHN.F.LUB326b & métapode & & Hipparion prostylum \\
\hline MNHN.F.LUB327 & métapode & & Hipparion prostylum \\
\hline MNHN.F.LUB328 & métapode & & Hipparion prostylum \\
\hline MNHN.F.LUB329 & métapode & & Hipparion prostylum \\
\hline MNHN.F.LUB330a & métapode & & Hipparion prostylum \\
\hline MNHN.F.LUB330b & métapode & & Hipparion prostylum \\
\hline MNHN.F.LUB331 & métapode & & Hipparion prostylum \\
\hline MNHN.F.LUB332 & métapode & & Hipparion prostylum \\
\hline MNHN.F.LUB333 & métapode & & Hipparion prostylum \\
\hline MNHN.F.LUB334 & métapode & & Hipparion prostylum \\
\hline MNHN.F.LUB335 & métapode & & Hipparion prostylum \\
\hline MNHN.F.LUB336 & phalange & & Hipparion prostylum \\
\hline MNHN.F.LUB337 & phalange & & Hipparion prostylum \\
\hline MNHN.F.LUB338 & phalange & & Hipparion prostylum \\
\hline MNHN.F.LUB339 & phalange & & Hipparion prostylum \\
\hline MNHN.F.LUB340 & phalange & & Hipparion prostylum \\
\hline MNHN.F.LUB341 & métapode & & Hipparion prostylum \\
\hline MNHN.F.LUB342 & métapode & & Hipparion prostylum \\
\hline MNHN.F.LUB343 & métapode & & Hipparion prostylum \\
\hline MNHN.F.LUB344 & phalange & & Hipparion prostylum \\
\hline MNHN.F.LUB345 & phalange & & Hipparion prostylum \\
\hline MNHN.F.LUB346 & métapode & & Hipparion prostylum \\
\hline MNHN.F.LUB347 & métapode & & Hipparion prostylum \\
\hline MNHN.F.LUB348 & métapode & & Hipparion prostylum \\
\hline MNHN.F.LUB349 & métapode & & Hipparion prostylum \\
\hline MNHN.F.LUB350 & phalange & & Hipparion prostylum \\
\hline MNHN.F.LUB351 & phalange & & Hipparion prostylum \\
\hline MNHN.F.LUB352 & phalange & & Hipparion prostylum \\
\hline MNHN.F.LUB353 & phalange & & Hipparion prostylum \\
\hline
\end{tabular}


ANNEXE 1. - Suite.

\begin{tabular}{|c|c|c|}
\hline Numéro de spécimen & Statut nomenclatural & Espèce \\
\hline MNHN.F.LUB354 & phalange & Hipparion prostylum \\
\hline MNHN.F.LUB355 & phalange & Hipparion prostylum \\
\hline MNHN.F.LUB356 & phalange & Hipparion prostylum \\
\hline MNHN.F.LUB357 & phalange & Hipparion prostylum \\
\hline MNHN.F.LUB358 & phalange & Hipparion prostylum \\
\hline MNHN.F.LUB359 & phalange & Hipparion prostylum \\
\hline MNHN.F.LUB360 & phalange & Hipparion prostylum \\
\hline MNHN.F.LUB361 & phalange & Hipparion prostylum \\
\hline MNHN.F.LUB362 & basipode & Hipparion prostylum \\
\hline MNHN.F.LUB363 & basipode & Hipparion prostylum \\
\hline MNHN.F.LUB364 & basipode & Hipparion prostylum \\
\hline MNHN.F.LUB365 & basipode & Hipparion prostylum \\
\hline LUB 366 & spécimen non retrouvé en collection & \\
\hline MNHN.F.LUB367 & basipode & Hipparion prostylum \\
\hline MNHN.F.LUB368 & basipode & Hipparion prostylum \\
\hline MNHN.F.LUB369 & basipode & Hipparion prostylum \\
\hline MNHN.F.LUB370 & basipode & Hipparion prostylum \\
\hline MNHN.F.LUB371 & basipode & Hipparion prostylum \\
\hline MNHN.F.LUB372 & sésamoïde & Hipparion prostylum \\
\hline MNHN.F.LUB373 & sésamoïde & Hipparion prostylum \\
\hline MNHN.F.LUB374 & sésamoïde & Hipparion prostylum \\
\hline MNHN.F.LUB375 & sésamoïde & Hipparion prostylum \\
\hline MNHN.F.LUB376 & sésamoïde & Hipparion prostylum \\
\hline MNHN.F.LUB377 & sésamoïde & Hipparion prostylum \\
\hline MNHN.F.LUB378 & sésamoïde & Hipparion prostylum \\
\hline MNHN.F.LUB379 & sésamoïde & Hipparion prostylum \\
\hline MNHN.F.LUB380 & sésamoïde & Hipparion prostylum \\
\hline MNHN.F.LUB381 & basipode & Hipparion prostylum \\
\hline MNHN.F.LUB382 & basipode & Hipparion prostylum \\
\hline MNHN.F.LUB383 & basipode & Hipparion prostylum \\
\hline MNHN.F.LUB384 & basipode & Hipparion prostylum \\
\hline MNHN.F.LUB385 & basipode & Hipparion prostylum \\
\hline MNHN.F.LUB386 & basipode & Hipparion prostylum \\
\hline MNHN.F.LUB387 & métapode & Hipparion prostylum \\
\hline MNHN.F.LUB388 & basipode & Hipparion prostylum \\
\hline MNHN.F.LUB389 & basipode & Hipparion prostylum \\
\hline MNHN.F.LUB390 & basipode & Hipparion prostylum \\
\hline MNHN.F.LUB391 & métapode & Hipparion prostylum \\
\hline MNHN.F.LUB392 & métapode & Hipparion prostylum \\
\hline MNHN.F.LUB393 & métapode & Hipparion prostylum \\
\hline MNHN.F.LUB394 & métapode & Hipparion prostylum \\
\hline MNHN.F.LUB395 & métapode & Hipparion prostylum \\
\hline MNHN.F.LUB396 & métapode & Hipparion prostylum \\
\hline MNHN.F.LUB397 & métapode & Hipparion prostylum \\
\hline MNHN.F.LUB398 & métapode & Hipparion prostylum \\
\hline MNHN.F.LUB399 & basipode & Hipparion prostylum \\
\hline MNHN.F.LUB400 & basipode & Hipparion prostylum \\
\hline MNHN.F.LUB401a & basipode & Hipparion prostylum \\
\hline MNHN.F.LUB401b & basipode & Hipparion prostylum \\
\hline MNHN.F.LUB401c & basipode & Hipparion prostylum \\
\hline MNHN.F.LUB402 & basipode & Hipparion prostylum \\
\hline MNHN.F.LUB403a & basipode & Hipparion prostylum \\
\hline MNHN.F.LUB403b & basipode & Hipparion prostylum \\
\hline MNHN.F.LUB404 & basipode & Hipparion prostylum \\
\hline MNHN.F.LUB405 & basipode & Hipparion prostylum \\
\hline MNHN.F.LUB406 & basipode & Hipparion prostylum \\
\hline
\end{tabular}


ANNEXE 1. - Suite.

\begin{tabular}{|c|c|c|c|}
\hline Numéro de spécimen & Anatomie & Statut nomenclatural & Espèce \\
\hline MNHN.F.LUB407 & phalange & & Hipparion prostylum \\
\hline MNHN.F.LUB408 & phalange & & Hipparion prostylum \\
\hline MNHN.F.LUB409 & phalange & & Hipparion prostylum \\
\hline MNHN.F.LUB410 & phalange & & Hipparion prostylum \\
\hline MNHN.F.LUB411 & phalange & & Hipparion prostylum \\
\hline MNHN.F.LUB412 & phalange & & Hipparion prostylum \\
\hline MNHN.F.LUB413 & phalange & & Hipparion prostylum \\
\hline MNHN.F.LUB414 & métapode & & Hipparion prostylum \\
\hline MNHN.F.LUB415 & métapode & & Hipparion prostylum \\
\hline MNHN.F.LUB416 & métapode & & Hipparion prostylum \\
\hline MNHN.F.LUB417 & métapode & & Hipparion prostylum \\
\hline MNHN.F.LUB418 & basipode & & Hipparion prostylum \\
\hline MNHN.F.LUB419 & basipode & & Hipparion prostylum \\
\hline MNHN.F.LUB420a & métapode & & Hipparion prostylum \\
\hline MNHN.F.LUB420b & métapode & & Hipparion prostylum \\
\hline MNHN.F.LUB420c & métapode & & Hipparion prostylum \\
\hline MNHN.F.LUB421 & métapode & & Hipparion prostylum \\
\hline MNHN.F.LUB422 & os indet. & & Incertae sedis \\
\hline MNHN.F.LUB423 & zeugopode & & Hipparion prostylum \\
\hline MNHN.F.LUB424 & zeugopode & & Hipparion prostylum \\
\hline MNHN.F.LUB425 & zeugopode & & Hipparion prostylum \\
\hline MNHN.F.LUB426 & phalange & & Hipparion prostylum \\
\hline MNHN.F.LUB427 & basipode & & Hipparion prostylum \\
\hline MNHN.F.LUB428 & phalange & & Hipparion prostylum \\
\hline MNHN.F.LUB429 & basipode & & Hipparion prostylum \\
\hline MNHN.F.LUB430 & basipode & & Hipparion prostylum \\
\hline MNHN.F.LUB431 & basipode & & Hipparion prostylum \\
\hline MNHN.F.LUB432 & métapode & & Hipparion prostylum \\
\hline MNHN.F.LUB433 & métapode & & Hipparion prostylum \\
\hline MNHN.F.LUB434 & métapode & & Hipparion prostylum \\
\hline MNHN.F.LUB435 & métapode & & Hipparion prostylum \\
\hline MNHN.F.LUB436 & zeugopode & & Hipparion prostylum \\
\hline MNHN.F.LUB437 & zeugopode & & Hipparion prostylum \\
\hline MNHN.F.LUB438a & zeugopode & & Hipparion prostylum \\
\hline MNHN.F.LUB438b & zeugopode & & Hipparion prostylum \\
\hline MNHN.F.LUB439 & zeugopode & & Hipparion prostylum \\
\hline MNHN.F.LUB440a & zeugopode & & Hipparion prostylum \\
\hline MNHN.F.LUB440b & basipode & & Hipparion prostylum \\
\hline MNHN.F.LUB440c & basipode & & Hipparion prostylum \\
\hline MNHN.F.LUB440d & basipode & & Hipparion prostylum \\
\hline MNHN.F.LUB440e & basipode & & Hipparion prostylum \\
\hline MNHN.F.LUB441a & zeugopode & & Hipparion prostylum \\
\hline MNHN.F.LUB441b & zeugopode & & Hipparion prostylum \\
\hline MNHN.F.LUB442a & zeugopode & & Hipparion prostylum \\
\hline MNHN.F.LUB442b & zeugopode & & Hipparion prostylum \\
\hline MNHN.F.LUB443 & basipode & & Hipparion prostylum \\
\hline MNHN.F.LUB444 & métapode & & Hipparion prostylum \\
\hline MNHN.F.LUB445 & basipode & & Hipparion prostylum \\
\hline MNHN.F.LUB446 & stylopode & & Hipparion prostylum \\
\hline MNHN.F.LUB447 & zeugopode & & Hipparion prostylum \\
\hline MNHN.F.LUB448 & stylopode & & Hipparion prostylum \\
\hline MNHN.F.LUB449 & zeugopode & & Hipparion prostylum \\
\hline MNHN.F.LUB450 & stylopode & & Hipparion prostylum \\
\hline MNHN.F.LUB451 & crâne & FIGURÉ & Hipparion prostylum \\
\hline MNHN.F.LUB452 & mandibule & FIGURÉ & Hipparion prostylum \\
\hline MNHN.F.LUB453 & mandibule & FIGURÉ & Hipparion prostylum \\
\hline
\end{tabular}


ANNEXE 1. - Suite.

\begin{tabular}{|c|c|c|c|}
\hline Numéro de spécimen & Anatomie & Statut nomenclatural & Espèce \\
\hline MNHN.F.LUB454 & crâne & FIGURÉ & Hipparion prostylum \\
\hline MNHN.F.LUB455 & crâne & & Hipparion prostylum \\
\hline MNHN.F.LUB456 & crâne & FIGURÉ & Gazella deperdita \\
\hline MNHN.F.LUB457 & mandibule & FIGURÉ & Hipparion prostylum \\
\hline MNHN.F.LUB458 & dentition supérieure & & Hipparion prostylum \\
\hline MNHN.F.LUB459 & métapode & FIGURÉ & Hipparion prostylum \\
\hline MNHN.F.LUB460 & métapode & FIGURÉ & Hipparion prostylum \\
\hline LUD 461 & basipode & FIGURÉ & Hipparion prostylum \\
\hline MNHN.F.LUB462 & basipode & & Hipparion prostylum \\
\hline MNHN.F.LUB463 & crâne & FIGURÉ & Gazella deperdita \\
\hline MNHN.F.LUB464 & crâne & & Gazella deperdita \\
\hline MNHN.F.LUB465 & phalange & FIGURÉ & Hipparion prostylum \\
\hline MNHN.F.LUB466 & phalange & FIGURÉ & Hipparion prostylum \\
\hline MNHN.F.LUB467 & mandibule & & Hipparion prostylum \\
\hline MNHN.F.LUB468 & crâne & & Gazella deperdita \\
\hline MNHN.F.LUB469 & crâne & & Gazella deperdita \\
\hline MNHN.F.LUB470 & crâne & & Gazella deperdita \\
\hline MNHN.F.LUB471 & crâne & & Gazella deperdita \\
\hline MNHN.F.LUB472 & crâne & & Gazella deperdita \\
\hline MNHN.F.LUB473 & crâne & & Gazella deperdita \\
\hline MNHN.F.LUB474 & crâne & & Gazella deperdita \\
\hline MNHN.F.LUB475 & crâne & & Gazella deperdita \\
\hline MNHN.F.LUB476 & crâne & & Gazella deperdita \\
\hline MNHN.F.LUB477 & crâne & & Gazella deperdita \\
\hline MNHN.F.LUB478 & crâne & & Gazella deperdita \\
\hline MNHN.F.LUB479 & crâne & & Gazella deperdita \\
\hline MNHN.F.LUB480 & crâne & & Gazella deperdita \\
\hline MNHN.F.LUB481 & crâne & & Gazella deperdita \\
\hline MNHN.F.LUB482 & crâne & & Gazella deperdita \\
\hline MNHN.F.LUB483 & crâne & & Gazella deperdita \\
\hline MNHN.F.LUB484 & crâne & & Gazella deperdita \\
\hline MNHN.F.LUB485 & crâne & & Gazella deperdita \\
\hline MNHN.F.LUB486 & crâne & & Gazella deperdita \\
\hline MNHN.F.LUB487 & crâne & & Gazella deperdita \\
\hline MNHN.F.LUB488 & crâne & & Incertae sedis \\
\hline MNHN.F.LUB489 & crâne & & Incertae sedis \\
\hline MNHN.F.LUB490 & crâne & & Incertae sedis \\
\hline MNHN.F.LUB491a & basipode & & Gazella deperdita \\
\hline MNHN.F.LUB491b & métapode & & Gazella deperdita \\
\hline MNHN.F.LUB491c & os indet. & & Gazella deperdita \\
\hline MNHN.F.LUB491d & basipode & & Gazella deperdita \\
\hline MNHN.F.LUB491e & phalange & & Gazella deperdita \\
\hline MNHN.F.LUB492a & zeugopode & FIGURÉ & Gazella deperdita \\
\hline MNHN.F.LUB492b & basipode & FIGURÉ & Gazella deperdita \\
\hline MNHN.F.LUB492c & zeugopode & FIGURÉ & Gazella deperdita \\
\hline MNHN.F.LUB493a & basipode & & Gazella deperdita \\
\hline MNHN.F.LUB493b & basipode & & Gazella deperdita \\
\hline MNHN.F.LUB494 & basipode & & Gazella deperdita \\
\hline MNHN.F.LUB495 & basipode & & Gazella deperdita \\
\hline MNHN.F.LUB496 & basipode & & Gazella deperdita \\
\hline MNHN.F.LUB497a & basipode & & Gazella deperdita \\
\hline MNHN.F.LUB497b & basipode & & Gazella deperdita \\
\hline MNHN.F.LUB498 & basipode & & Gazella deperdita \\
\hline MNHN.F.LUB499 & basipode & & Gazella deperdita \\
\hline MNHN.F.LUB500 & basipode & & Gazella deperdita \\
\hline MNHN.F.LUB501 & basipode & & Gazella deperdita \\
\hline
\end{tabular}


ANNEXE 1. - Suite.

\begin{tabular}{|c|c|c|c|}
\hline Numéro de spécimen & Anatomie & Statut nomenclatural & Espèce \\
\hline MNHN.F.LUB502 & basipode & & Gazella deperdita \\
\hline MNHN.F.LUB503 & basipode & & Incertae sedis \\
\hline MNHN.F.LUB504 & basipode & & Gazella deperdita \\
\hline MNHN.F.LUB505 & basipode & & Gazella deperdita \\
\hline MNHN.F.LUB506 & os indet. & & Incertae sedis \\
\hline MNHN.F.LUB507 & mandibule & & Hipparion prostylum \\
\hline MNHN.F.LUB508 & crâne & & Gazella deperdita \\
\hline MNHN.F.LUB509a & crâne & & Gazella deperdita \\
\hline MNHN.F.LUB509b & crâne & & Gazella deperdita \\
\hline MNHN.F.LUB509c & crâne & & Gazella deperdita \\
\hline MNHN.F.LUB509d & crâne & & Gazella deperdita \\
\hline MNHN.F.LUB510 & crâne & NÉOTYPE & Gazella deperdita \\
\hline MNHN.F.LUB511 & mandibule & & Gazella deperdita \\
\hline MNHN.F.LUB512 & mandibule & FIGURÉ & Gazella deperdita \\
\hline MNHN.F.LUB513 & dentition inférieure & FIGURÉ & Gazella deperdita \\
\hline MNHN.F.LUB514 & dentition inférieure & FIGURÉ & Gazella deperdita \\
\hline MNHN.F.LUB515 & crâne & PARATYPE & Gazella deperdita \\
\hline MNHN.F.LUB516 & crâne & FIGURÉ & Gazella deperdita \\
\hline MNHN.F.LUB517 & crâne & FIGURÉ & Gazella deperdita \\
\hline MNHN.F.LUB518 & crâne & FIGURÉ & Gazella deperdita \\
\hline MNHN.F.LUB519 & crâne & FIGURÉ & Gazella deperdita \\
\hline MNHN.F.LUB520 & crâne & FIGURÉ & Gazella deperdita \\
\hline MNHN.F.LUB521 & crâne & FIGURÉ & Gazella deperdita \\
\hline MNHN.F.LUB522 & crâne & FIGURÉ & Gazella deperdita \\
\hline MNHN.F.LUB523 & crâne & & Gazella deperdita \\
\hline MNHN.F.LUB524 & crâne & FIGURÉ & Gazella deperdita \\
\hline MNHN.F.LUB525 & mandibule & & Gazella deperdita \\
\hline MNHN.F.LUB526 & mandibule & & Gazella deperdita \\
\hline MNHN.F.LUB527 & dentition inférieure & FIGURÉ & Gazella deperdita \\
\hline MNHN.F.LUB528 & mandibule & & Gazella deperdita \\
\hline MNHN.F.LUB529 & mandibule & & Gazella deperdita \\
\hline MNHN.F.LUB530 & mandibule & & Gazella deperdita \\
\hline MNHN.F.LUB531 & mandibule & & Gazella deperdita \\
\hline MNHN.F.LUB532 & mandibule & & Gazella deperdita \\
\hline MNHN.F.LUB533 & mandibule & & Gazella deperdita \\
\hline MNHN.F.LUB534 & mandibule & & Gazella deperdita \\
\hline MNHN.F.LUB535 & mandibule & & Gazella deperdita \\
\hline MNHN.F.LUB536 & mandibule & & Gazella deperdita \\
\hline MNHN.F.LUB537 & mandibule & & Gazella deperdita \\
\hline MNHN.F.LUB538 & mandibule & & Gazella deperdita \\
\hline MNHN.F.LUB539 & mandibule & & Gazella deperdita \\
\hline MNHN.F.LUB540 & mandibule & & Gazella deperdita \\
\hline MNHN.F.LUB541 & dentition inférieure & & Gazella deperdita \\
\hline MNHN.F.LUB542 & mandibule & & Gazella deperdita \\
\hline MNHN.F.LUB543 & mandibule & & Gazella deperdita \\
\hline MNHN.F.LUB544 & mandibule & & Gazella deperdita \\
\hline MNHN.F.LUB545 & mandibule & & Gazella deperdita \\
\hline MNHN.F.LUB546 & mandibule & FIGURÉ & Gazella deperdita \\
\hline MNHN.F.LUB547 & mandibule & & Gazella deperdita \\
\hline MNHN.F.LUB548 & mandibule & & Gazella deperdita \\
\hline MNHN.F.LUB549 & mandibule & & Gazella deperdita \\
\hline MNHN.F.LUB550 & crâne & & Gazella deperdita \\
\hline MNHN.F.LUB551 & crâne & & Incertae sedis \\
\hline MNHN.F.LUB552 & dentition supérieure & & Gazella deperdita \\
\hline MNHN.F.LUB553 & crâne & & Gazella deperdita \\
\hline MNHN.F.LUB554 & crâne & FIGURÉ & Gazella deperdita \\
\hline
\end{tabular}


ANNEXE 1. - Suite.

\begin{tabular}{|c|c|c|c|}
\hline Numéro de spécimen & Anatomie & Statut nomenclatural & Espèce \\
\hline MNHN.F.LUB555 & crâne & & Gazella deperdita \\
\hline MNHN.F.LUB556 & crâne & & Gazella deperdita \\
\hline MNHN.F.LUB557 & crâne & & Gazella deperdita \\
\hline MNHN.F.LUB558 & stylopode & FIGURÉ & Gazella deperdita \\
\hline MNHN.F.LUB559 & zeugopode & & Gazella deperdita \\
\hline MNHN.F.LUB560a & stylopode & & Gazella deperdita \\
\hline MNHN.F.LUB560b & zeugopode & & Gazella deperdita \\
\hline MNHN.F.LUB561a & stylopode & & Gazella deperdita \\
\hline MNHN.F.LUB561b & zeugopode & & Gazella deperdita \\
\hline MNHN.F.LUB561c & zeugopode & & Gazella deperdita \\
\hline MNHN.F.LUB562 & stylopode & & Gazella deperdita \\
\hline MNHN.F.LUB563 & stylopode & & Gazella deperdita \\
\hline MNHN.F.LUB564 & stylopode & FIGURÉ & Gazella deperdita \\
\hline MNHN.F.LUB565 & stylopode & & Gazella deperdita \\
\hline MNHN.F.LUB566 & stylopode & & Gazella deperdita \\
\hline MNHN.F.LUB567 & stylopode & & Gazella deperdita \\
\hline MNHN.F.LUB568 & zeugopode & & Gazella deperdita \\
\hline MNHN.F.LUB569 & zeugopode & & Gazella deperdita \\
\hline MNHN.F.LUB570 & zeugopode & & Gazella deperdita \\
\hline MNHN.F.LUB571 & zeugopode & & Gazella deperdita \\
\hline MNHN.F.LUB572 & os indet. & & Gazella deperdita \\
\hline MNHN.F.LUB573 & zeugopode & & Gazella deperdita \\
\hline MNHN.F.LUB574 & zeugopode & FIGURÉ & Gazella deperdita \\
\hline MNHN.F.LUB575 & zeugopode & & Gazella deperdita \\
\hline MNHN.F.LUB576 & zeugopode & & Gazella deperdita \\
\hline MNHN.F.LUB577 & ceinture & & Gazella deperdita \\
\hline MNHN.F.LUB578 & zeugopode & & Gazella deperdita \\
\hline MNHN.F.LUB579 & zeugopode & & Gazella deperdita \\
\hline MNHN.F.LUB580 & zeugopode & & Gazella deperdita \\
\hline MNHN.F.LUB581 & phalange & & Gazella deperdita \\
\hline MNHN.F.LUB582 & phalange & & Gazella deperdita \\
\hline MNHN.F.LUB583 & phalange & & Gazella deperdita \\
\hline MNHN.F.LUB584 & phalange & & Gazella deperdita \\
\hline MNHN.F.LUB585 & phalange & & Gazella deperdita \\
\hline MNHN.F.LUB586 & phalange & & Gazella deperdita \\
\hline MNHN.F.LUB587 & phalange & FIGURÉ & Gazella deperdita \\
\hline MNHN.F.LUB588 & phalange & & Gazella deperdita \\
\hline MNHN.F.LUB589 & phalange & & Gazella deperdita \\
\hline MNHN.F.LUB590 & phalange & & Gazella deperdita \\
\hline MNHN.F.LUB591 & phalange & & Gazella deperdita \\
\hline MNHN.F.LUB592 & phalange & & Gazella deperdita \\
\hline MNHN.F.LUB593 & phalange & & Gazella deperdita \\
\hline MNHN.F.LUB594 & phalange & FIGURÉ & Gazella deperdita \\
\hline MNHN.F.LUB595 & phalange & & Gazella deperdita \\
\hline MNHN.F.LUB596 & phalange & & Gazella deperdita \\
\hline MNHN.F.LUB597 & phalange & & Gazella deperdita \\
\hline MNHN.F.LUB598 & phalange & & Gazella deperdita \\
\hline MNHN.F.LUB599 & phalange & & Gazella deperdita \\
\hline MNHN.F.LUB600 & phalange & & Gazella deperdita \\
\hline MNHN.F.LUB601 & phalange & & Gazella deperdita \\
\hline MNHN.F.LUB602 & phalange & FIGURÉ & Gazella deperdita \\
\hline MNHN.F.LUB603 & phalange & & Gazella deperdita \\
\hline MNHN.F.LUB604 & basipode & & Gazella deperdita \\
\hline MNHN.F.LUB605 & basipode & & Gazella deperdita \\
\hline MNHN.F.LUB606 & zeugopode & & Gazella deperdita \\
\hline MNHN.F.LUB607 & basipode & & Gazella deperdita \\
\hline
\end{tabular}


ANNEXE 1. - Suite.

\begin{tabular}{|c|c|c|c|}
\hline Numéro de spécimen & Anatomie & Statut nomenclatural & Espèce \\
\hline MNHN.F.LUB608 & basipode & & Gazella deperdita \\
\hline MNHN.F.LUB609 & basipode & & Gazella deperdita \\
\hline MNHN.F.LUB610 & basipode & & Gazella deperdita \\
\hline MNHN.F.LUB611 & zeugopode & & Gazella deperdita \\
\hline MNHN.F.LUB612 & basipode & & Gazella deperdita \\
\hline MNHN.F.LUB613 & basipode & FIGURÉ & Gazella deperdita \\
\hline MNHN.F.LUB614 & basipode & & Gazella deperdita \\
\hline MNHN.F.LUB615 & os indet. & & Incertae sedis \\
\hline MNHN.F.LUB616 & basipode & & Gazella deperdita \\
\hline MNHN.F.LUB617a & basipode & & Gazella deperdita \\
\hline MNHN.F.LUB617b & basipode & & Gazella deperdita \\
\hline MNHN.F.LUB618a & basipode & FIGURÉ & Gazella deperdita \\
\hline MNHN.F.LUB618b & basipode & FIGURÉ & Gazella deperdita \\
\hline MNHN.F.LUB619 & basipode & & Gazella deperdita \\
\hline MNHN.F.LUB620 & stylopode & & Gazella deperdita \\
\hline MNHN.F.LUB621 & stylopode & & Gazella deperdita \\
\hline MNHN.F.LUB622 & stylopode & & Gazella deperdita \\
\hline MNHN.F.LUB623 & vertèbre & & Gazella deperdita \\
\hline MNHN.F.LUB624 & stylopode & & Gazella deperdita \\
\hline MNHN.F.LUB625 & vertèbre & & Gazella deperdita \\
\hline MNHN.F.LUB626 & os indet. & & Incertae sedis \\
\hline MNHN.F.LUB627 & stylopode & & Gazella deperdita \\
\hline MNHN.F.LUB628 & vertèbre & & Gazella deperdita \\
\hline MNHN.F.LUB629 & ceinture & & Gazella deperdita \\
\hline MNHN.F.LUB630 & vertèbre & & Gazella deperdita \\
\hline MNHN.F.LUB631 & métapode & & Gazella deperdita \\
\hline MNHN.F.LUB632 & métapode & & Gazella deperdita \\
\hline MNHN.F.LUB633 & métapode & FIGURÉ & Gazella deperdita \\
\hline MNHN.F.LUB634 & métapode & FIGURÉ & Gazella deperdita \\
\hline MNHN.F.LUB635 & métapode & & Gazella deperdita \\
\hline MNHN.F.LUB636 & métapode & & Gazella deperdita \\
\hline MNHN.F.LUB637 & métapode & & Gazella deperdita \\
\hline MNHN.F.LUB638 & métapode & & Gazella deperdita \\
\hline MNHN.F.LUB639 & métapode & & Gazella deperdita \\
\hline MNHN.F.LUB640 & métapode & & Gazella deperdita \\
\hline MNHN.F.LUB641 & métapode & & Gazella deperdita \\
\hline MNHN.F.LUB642 & métapode & & Gazella deperdita \\
\hline MNHN.F.LUB643 & métapode & FIGURÉ & Gazella deperdita \\
\hline MNHN.F.LUB644 & métapode & & Gazella deperdita \\
\hline MNHN.F.LUB645 & métapode & & Gazella deperdita \\
\hline MNHN.F.LUB646 & métapode & & Gazella deperdita \\
\hline MNHN.F.LUB647 & métapode & & Gazella deperdita \\
\hline MNHN.F.LUB648 & métapode & & Gazella deperdita \\
\hline MNHN.F.LUB649 & métapode & & Gazella deperdita \\
\hline MNHN.F.LUB650 & métapode & FIGURÉ & Gazella deperdita \\
\hline MNHN.F.LUB651 & métapode & & Gazella deperdita \\
\hline MNHN.F.LUB652 & métapode & & Gazella deperdita \\
\hline MNHN.F.LUB653 & métapode & & Gazella deperdita \\
\hline MNHN.F.LUB654 & métapode & & Gazella deperdita \\
\hline MNHN.F.LUB655 & crâne & & Gazella deperdita \\
\hline MNHN.F.LUB656 & mandibule & FIGURÉ & Microstonyx major \\
\hline MNHN.F.LUB657 & mandibule & & Microstonyx major \\
\hline MNHN.F.LUB658 & mandibule & & Microstonyx major \\
\hline MNHN.F.LUB659 & mandibule & FIGURÉ & Microstonyx major \\
\hline MNHN.F.LUB660 & crâne & FIGURÉ & Microstonyx major \\
\hline MNHN.F.LUB661 & crâne & & Microstonyx major \\
\hline
\end{tabular}


ANNEXE 1. - Suite.

\begin{tabular}{|c|c|c|c|}
\hline Numéro de spécimen & Anatomie & Statut nomenclatural & Espèce \\
\hline MNHN.F.LUB662 & crâne & FIGURÉ & Microstonyx major \\
\hline MNHN.F.LUB663 & zeugopode & FIGURÉ & Microstonyx major \\
\hline MNHN.F.LUB664 & stylopode & FIGURÉ & Microstonyx major \\
\hline MNHN.F.LUB665 & stylopode & & Microstonyx major \\
\hline MNHN.F.LUB666 & dentition inférieure & & Microstonyx major \\
\hline MNHN.F.LUB667 & dentition inférieure & & Microstonyx major \\
\hline MNHN.F.LUB668a & vertèbre & FIGURÉ & Microstonyx major \\
\hline MNHN.F.LUB668b & vertèbre & FIGURÉ & Microstonyx major \\
\hline MNHN.F.LUB668c & vertèbre & FIGURÉ & Microstonyx major \\
\hline MNHN.F.LUB669 & mandibule & & Gazella deperdita \\
\hline MNHN.F.LUB670 & dentition inférieure & & Gazella deperdita \\
\hline MNHN.F.LUB671 & dentition inférieure & & Gazella deperdita \\
\hline MNHN.F.LUB672 & dentition inférieure & & Gazella deperdita \\
\hline MNHN.F.LUB673 & dentition inférieure & & Gazella deperdita \\
\hline MNHN.F.LUB674 & dentition inférieure & & Gazella deperdita \\
\hline MNHN.F.LUB675 & dentition inférieure & & Gazella deperdita \\
\hline MNHN.F.LUB676 & dentition inférieure & & Gazella deperdita \\
\hline MNHN.F.LUB677 & dentition inférieure & & Gazella deperdita \\
\hline MNHN.F.LUB678 & dentition supérieure & & Gazella deperdita \\
\hline MNHN.F.LUB679 & dentition supérieure & & Gazella deperdita \\
\hline MNHN.F.LUB680 & dentition supérieure & & Gazella deperdita \\
\hline MNHN.F.LUB681 & crâne & FIGURÉ & Gazella deperdita \\
\hline MNHN.F.LUB682 & crâne & & Gazella deperdita \\
\hline MNHN.F.LUB683 & crâne & & Gazella deperdita \\
\hline MNHN.F.LUB684 & crâne & & Gazella deperdita \\
\hline MNHN.F.LUB685 & crâne & & Gazella deperdita \\
\hline MNHN.F.LUB686 & crâne & & Gazella deperdita \\
\hline MNHN.F.LUB687 & crâne & & Gazella deperdita \\
\hline MNHN.F.LUB688 & crâne & & Gazella deperdita \\
\hline MNHN.F.LUB689a & crâne & & Gazella deperdita \\
\hline MNHN.F.LUB689b & crâne & & Gazella deperdita \\
\hline MNHN.F.LUB690 & crâne & & Gazella deperdita \\
\hline MNHN.F.LUB691a & zeugopode & & Gazella deperdita \\
\hline MNHN.F.LUB691b & basipode & & Gazella deperdita \\
\hline MNHN.F.LUB691c & basipode & & Gazella deperdita \\
\hline MNHN.F.LUB691d & basipode & & Gazella deperdita \\
\hline MNHN.F.LUB691e & basipode & & Gazella deperdita \\
\hline MNHN.F.LUB691f & basipode & & Gazella deperdita \\
\hline MNHN.F.LUB691g & basipode & & Gazella deperdita \\
\hline MNHN.F.LUB691h & métapode & & Gazella deperdita \\
\hline MNHN.F.LUB692 & métapode & & Gazella deperdita \\
\hline MNHN.F.LUB693a & vertèbre & & Gazella deperdita \\
\hline MNHN.F.LUB693b & vertèbre & & Gazella deperdita \\
\hline MNHN.F.LUB693c & vertèbre & & Gazella deperdita \\
\hline MNHN.F.LUB694 & basipode & & Gazella deperdita \\
\hline MNHN.F.LUB695 & basipode & & Gazella deperdita \\
\hline MNHN.F.LUB696 & sésamoïde & & Gazella deperdita \\
\hline MNHN.F.LUB697 & sésamoïde & & Gazella deperdita \\
\hline MNHN.F.LUB698 & crâne & & Tragoportax amalthea \\
\hline MNHN.F.LUB699 & crâne & & Tragoportax amalthea \\
\hline MNHN.F.LUB700 & crâne & FIGURÉ & Tragoportax amalthea \\
\hline MNHN.F.LUB701 & crâne & FIGURÉ & Tragoportax amalthea \\
\hline MNHN.F.LUB702 & crâne & & Tragoportax amalthea \\
\hline MNHN.F.LUB703 & crâne & & Tragoportax amalthea \\
\hline MNHN.F.LUB704 & crâne & & Tragoportax amalthea \\
\hline MNHN.F.LUB705 & crâne & & Tragoportax amalthea \\
\hline
\end{tabular}


ANNEXE 1. - Suite.

\begin{tabular}{|c|c|c|c|}
\hline Numéro de spécimen & Anatomie & Statut nomenclatural & Espèce \\
\hline MNHN.F.LUB706 & crâne & & Tragoportax amalthea \\
\hline MNHN.F.LUB707 & crâne & & Tragoportax amalthea \\
\hline MNHN.F.LUB708 & crâne & & Tragoportax amalthea \\
\hline MNHN.F.LUB709 & crâne & & Tragoportax amalthea \\
\hline MNHN.F.LUB710 & crâne & & Tragoportax amalthea \\
\hline MNHN.F.LUB711 & crâne & & Tragoportax amalthea \\
\hline MNHN.F.LUB712 & crâne & & Tragoportax amalthea \\
\hline MNHN.F.LUB713 & crâne & & Tragoportax amalthea \\
\hline MNHN.F.LUB714 & crâne & & Tragoportax amalthea \\
\hline MNHN.F.LUB715 & crâne & FIGURÉ & Tragoportax amalthea \\
\hline MNHN.F.LUB716 & crâne & & Tragoportax amalthea \\
\hline MNHN.F.LUB717 & crâne & & Tragoportax amalthea \\
\hline MNHN.F.LUB718 & crâne & & Tragoportax amalthea \\
\hline MNHN.F.LUB719 & crâne & FIGURÉ & Tragoportax amalthea \\
\hline MNHN.F.LUB720 & crâne & FIGURÉ & Tragoportax amalthea \\
\hline MNHN.F.LUB721 & crâne & & Tragoportax amalthea \\
\hline MNHN.F.LUB722 & dentition supérieure & & Tragoportax amalthea \\
\hline MNHN.F.LUB723 & dentition supérieure & & Tragoportax amalthea \\
\hline MNHN.F.LUB724 & dentition supérieure & & Tragoportax amalthea \\
\hline MNHN.F.LUB725 & crâne & & Tragoportax amalthea \\
\hline MNHN.F.LUB726 & dentition supérieure & FIGURÉ & Tragoportax amalthea \\
\hline MNHN.F.LUB727 & dentition supérieure & & Tragoportax amalthea \\
\hline MNHN.F.LUB728 & dentition supérieure & & Tragoportax amalthea \\
\hline MNHN.F.LUB729 & dentition supérieure & FIGURÉ & Tragoportax amalthea \\
\hline MNHN.F.LUB730a & crâne & & Tragoportax amalthea \\
\hline MNHN.F.LUB730b & crâne & & Tragoportax amalthea \\
\hline MNHN.F.LUB731 & mandibule & & Tragoportax amalthea \\
\hline MNHN.F.LUB732 & mandibule & & Tragoportax amalthea \\
\hline MNHN.F.LUB733 & mandibule & & Tragoportax amalthea \\
\hline MNHN.F.LUB734 & mandibule & & Tragoportax amalthea \\
\hline MNHN.F.LUB735 & mandibule & & Tragoportax amalthea \\
\hline MNHN.F.LUB736 & mandibule & & Tragoportax amalthea \\
\hline MNHN.F.LUB737 & mandibule & & Tragoportax amalthea \\
\hline MNHN.F.LUB738 & mandibule & & Tragoportax amalthea \\
\hline MNHN.F.LUB739 & mandibule & & Tragoportax amalthea \\
\hline MNHN.F.LUB740 & mandibule & & Tragoportax amalthea \\
\hline MNHN.F.LUB741 & mandibule & & Tragoportax amalthea \\
\hline MNHN.F.LUB742 & mandibule & & Tragoportax amalthea \\
\hline MNHN.F.LUB743 & mandibule & & Tragoportax amalthea \\
\hline MNHN.F.LUB744 & mandibule & & Tragoportax amalthea \\
\hline MNHN.F.LUB745 & mandibule & & Tragoportax amalthea \\
\hline MNHN.F.LUB746 & mandibule & & Tragoportax amalthea \\
\hline MNHN.F.LUB747 & mandibule & & Tragoportax amalthea \\
\hline MNHN.F.LUB748 & dentition inférieure & & Tragoportax amalthea \\
\hline MNHN.F.LUB749 & dentition inférieure & FIGURÉ & Tragoportax amalthea \\
\hline MNHN.F.LUB750 & dentition inférieure & & Tragoportax amalthea \\
\hline MNHN.F.LUB751 & dentition inférieure & FIGURÉ & Tragoportax amalthea \\
\hline MNHN.F.LUB752 & dentition inférieure & & Tragoportax amalthea \\
\hline MNHN.F.LUB753 & dentition inférieure & & Tragoportax amalthea \\
\hline MNHN.F.LUB754 & stylopode & & Tragoportax amalthea \\
\hline MNHN.F.LUB755 & zeugopode & & Pliocervus matheroni \\
\hline MNHN.F.LUB756a & ceinture & & Tragoportax amalthea \\
\hline MNHN.F.LUB756b & ceinture & & Tragoportax amalthea \\
\hline MNHN.F.LUB757 & vertèbre & & Tragoportax amalthea \\
\hline MNHN.F.LUB758a & vertèbre & & Tragoportax amalthea \\
\hline MNHN.F.LUB758b & vertèbre & & Tragoportax amalthea \\
\hline
\end{tabular}


ANNEXE 1. - Suite.

\begin{tabular}{|c|c|c|c|}
\hline Numéro de spécimen & Anatomie & Statut nomenclatural & Espèce \\
\hline MNHN.F.LUB758c & vertèbre & & Tragoportax amalthea \\
\hline MNHN.F.LUB759 & vertèbre & & Tragoportax amalthea \\
\hline MNHN.F.LUB760 & vertèbre & & Tragoportax amalthea \\
\hline MNHN.F.LUB761 & zeugopode & & Tragoportax amalthea \\
\hline MNHN.F.LUB762 & zeugopode & & Tragoportax amalthea \\
\hline MNHN.F.LUB763 & zeugopode & & Tragoportax amalthea \\
\hline MNHN.F.LUB764a & zeugopode & & Tragoportax amalthea \\
\hline MNHN.F.LUB764b & zeugopode & & Tragoportax amalthea \\
\hline MNHN.F.LUB764c & basipode & & Tragoportax amalthea \\
\hline MNHN.F.LUB764d & basipode & & Tragoportax amalthea \\
\hline MNHN.F.LUB765a & zeugopode & & Tragoportax amalthea \\
\hline MNHN.F.LUB765b & basipode & & Tragoportax amalthea \\
\hline MNHN.F.LUB765c & basipode & & Tragoportax amalthea \\
\hline MNHN.F.LUB765d & basipode & & Tragoportax amalthea \\
\hline MNHN.F.LUB765e & basipode & & Tragoportax amalthea \\
\hline MNHN.F.LUB766 & basipode & & Tragoportax amalthea \\
\hline MNHN.F.LUB767a & zeugopode & & Tragoportax amalthea \\
\hline MNHN.F.LUB767b & zeugopode & & Tragoportax amalthea \\
\hline MNHN.F.LUB767c & basipode & & Tragoportax amalthea \\
\hline MNHN.F.LUB767d & basipode & & Tragoportax amalthea \\
\hline MNHN.F.LUB767e & basipode & & Tragoportax amalthea \\
\hline MNHN.F.LUB767f & basipode & & Tragoportax amalthea \\
\hline MNHN.F.LUB767g & basipode & & Tragoportax amalthea \\
\hline MNHN.F.LUB767h & métapode & & Tragoportax amalthea \\
\hline MNHN.F.LUB767i & phalange & & Tragoportax amalthea \\
\hline MNHN.F.LUB767j & phalange & & Tragoportax amalthea \\
\hline MNHN.F.LUB768 & basipode & & Tragoportax amalthea \\
\hline MNHN.F.LUB769 & basipode & & Tragoportax amalthea \\
\hline MNHN.F.LUB770 & basipode & & Tragoportax amalthea \\
\hline MNHN.F.LUB771 & métapode & & Tragoportax amalthea \\
\hline MNHN.F.LUB772 & métapode & & Tragoportax amalthea \\
\hline MNHN.F.LUB773 & métapode & & Tragoportax amalthea \\
\hline MNHN.F.LUB774 & métapode & & Tragoportax amalthea \\
\hline MNHN.F.LUB775 & phalange & & Tragoportax amalthea \\
\hline MNHN.F.LUB776 & phalange & & Tragoportax amalthea \\
\hline MNHN.F.LUB777 & phalange & & Tragoportax amalthea \\
\hline MNHN.F.LUB778 & phalange & & Tragoportax amalthea \\
\hline MNHN.F.LUB779 & phalange & & Tragoportax amalthea \\
\hline MNHN.F.LUB780 & phalange & & Tragoportax amalthea \\
\hline MNHN.F.LUB781 & crâne & FIGURÉ & Pliocervus matheroni \\
\hline MNHN.F.LUB782 & crâne & FIGURÉ & Pliocervus matheroni \\
\hline MNHN.F.LUB783 & crâne & FIGURÉ & Pliocervus matheroni \\
\hline MNHN.F.LUB784 & crâne & & Pliocervus matheroni \\
\hline MNHN.F.LUB785 & crâne & & Pliocervus matheroni \\
\hline MNHN.F.LUB786 & crâne & & Pliocervus matheroni \\
\hline MNHN.F.LUB787 & crâne & & Pliocervus matheroni \\
\hline MNHN.F.LUB788a & crâne & & Pliocervus matheroni \\
\hline MNHN.F.LUB788b & crâne & & Pliocervus matheroni \\
\hline MNHN.F.LUB789 & crâne & & Pliocervus matheroni \\
\hline MNHN.F.LUB790 & crâne & & Pliocervus matheroni \\
\hline MNHN.F.LUB791 & crâne & & Pliocervus matheroni \\
\hline MNHN.F.LUB792 & crâne & & Pliocervus matheroni \\
\hline MNHN.F.LUB793 & crâne & & Pliocervus matheroni \\
\hline MNHN.F.LUB794 & crâne & & Pliocervus matheroni \\
\hline MNHN.F.LUB795a & crâne & & Pliocervus matheroni \\
\hline MNHN.F.LUB795b & crâne & & Pliocervus matheroni \\
\hline
\end{tabular}


ANNEXE 1. - Suite.

\begin{tabular}{|c|c|c|c|}
\hline Numéro de spécimen & Anatomie & Statut nomenclatural & Espèce \\
\hline MNHN.F.LUB796 & crâne & & Pliocervus matheroni \\
\hline MNHN.F.LUB797 & crâne & & Pliocervus matheroni \\
\hline MNHN.F.LUB798 & crâne & FIGURÉ & Pliocervus matheroni \\
\hline MNHN.F.LUB799 & mandibule & FIGURÉ & Pliocervus matheroni \\
\hline MNHN.F.LUB800 & mandibule & FIGURÉ & Adcrocuta eximia \\
\hline MNHN.F.LUB801 & dentition indéterminée & & Adcrocuta eximia \\
\hline MNHN.F.LUB802 & mandibule & FIGURÉ & Adcrocuta eximia \\
\hline MNHN.F.LUB803 & dentition inférieure & FIGURÉ & Adcrocuta eximia \\
\hline MNHN.F.LUB804 & mandibule & FIGURÉ & Adcrocuta eximia \\
\hline MNHN.F.LUB805 & dentition indéterminée & & Adcrocuta eximia \\
\hline MNHN.F.LUB806 & zeugopode & FIGURÉ & Amphimachairodus giganteus \\
\hline MNHN.F.LUB807 & mandibule & FIGURÉ & Amphimachairodus giganteus \\
\hline MNHN.F.LUB808 & dentition supérieure & FIGURÉ & Hyaenictitherium wongii \\
\hline MNHN.F.LUB809a & objet autre & FIGURÉ & Hyaenictitherium wongii \\
\hline MNHN.F.LUB809b & objet autre & FIGURÉ & Hyaenictitherium wongii \\
\hline MNHN.F.LUB809c & objet autre & FIGURÉ & Hyaenictitherium wongii \\
\hline MNHN.F.LUB810a & métapode & FIGURÉ & Plioviverrops orbignyi \\
\hline MNHN.F.LUB810b & métapode & FIGURÉ & Plioviverrops orbignyi \\
\hline MNHN.F.LUB810c & métapode & FIGURÉ & Plioviverrops orbignyi \\
\hline MNHN.F.LUB810d & métapode & FIGURÉ & Plioviverrops orbignyi \\
\hline MNHN.F.LUB811 & mandibule & & Pliocervus matheroni \\
\hline MNHN.F.LUB812 & dentition inférieure & & Pliocervus matheroni \\
\hline MNHN.F.LUB813 & dentition inférieure & & Pliocervus matheroni \\
\hline MNHN.F.LUB814 & dentition inférieure & & Pliocervus matheroni \\
\hline MNHN.F.LUB815 & vertèbre & & Pliocervus matheroni \\
\hline MNHN.F.LUB816 & phalange & & Pliocervus matheroni \\
\hline MNHN.F.LUB817 & dentition supérieure & & Incertae sedis \\
\hline MNHN.F.LUB818 & vertèbre & & Incertae sedis \\
\hline MNHN.F.LUB819 & zeugopode & & Helladotherium duvernoyi \\
\hline MNHN.F.LUB820 & zeugopode & FIGURÉ & Helladotherium duvernoyi \\
\hline MNHN.F.LUB821 & zeugopode & & Helladotherium duvernoyi \\
\hline MNHN.F.LUB822 & stylopode & & Helladotherium duvernoyi \\
\hline MNHN.F.LUB823 & zeugopode & & Helladotherium duvernoyi \\
\hline MNHN.F.LUB824 & métapode & FIGURÉ & Helladotherium duvernoyi \\
\hline MNHN.F.LUB825 & zeugopode & & Helladotherium duvernoyi \\
\hline MNHN.F.LUB826a & zeugopode & & Helladotherium duvernoyi \\
\hline MNHN.F.LUB826b & basipode & & Helladotherium duvernoyi \\
\hline MNHN.F.LUB827a & basipode & & Helladotherium duvernoyi \\
\hline MNHN.F.LUB827b & basipode & & Helladotherium duvernoyi \\
\hline MNHN.F.LUB827c & basipode & & Helladotherium duvernoyi \\
\hline MNHN.F.LUB827d & métapode & & Helladotherium duvernoyi \\
\hline MNHN.F.LUB828 & métapode & FIGURÉ & Helladotherium duvernoyi \\
\hline MNHN.F.LUB829 & métapode & & Helladotherium duvernoyi \\
\hline MNHN.F.LUB830 & métapode & & Helladotherium duvernoyi \\
\hline MNHN.F.LUB831 & métapode & & Helladotherium duvernoyi \\
\hline MNHN.F.LUB832 & métapode & & Helladotherium duvernoyi \\
\hline MNHN.F.LUB833 & métapode & & Helladotherium duvernoyi \\
\hline MNHN.F.LUB834 & métapode & & Helladotherium duvernoyi \\
\hline MNHN.F.LUB835 & métapode & & Helladotherium duvernoyi \\
\hline MNHN.F.LUB836 & métapode & & Helladotherium duvernoyi \\
\hline MNHN.F.LUB837 & crâne & & Hipparion prostylum \\
\hline MNHN.F.LUB838 & crâne & & Hipparion prostylum \\
\hline MNHN.F.LUB839 & crâne & & Hipparion prostylum \\
\hline MNHN.F.LUB840 & crâne & FIGURÉ & Hipparion prostylum \\
\hline MNHN.F.LUB841 & crâne & FIGURÉ & Hipparion prostylum \\
\hline MNHN.F.LUB842 & crâne & & Hipparion prostylum \\
\hline
\end{tabular}


ANNEXE 1. - Suite.

\begin{tabular}{|c|c|c|c|}
\hline Numéro de spécimen & Anatomie & Statut nomenclatural & Espèce \\
\hline MNHN.F.LUB843a & crâne & & Hipparion prostylum \\
\hline MNHN.F.LUB843b & mandibule & & Hipparion prostylum \\
\hline MNHN.F.LUB843c & mandibule & & Hipparion prostylum \\
\hline MNHN.F.LUB844 & crâne & & Hipparion prostylum \\
\hline MNHN.F.LUB845 & crâne & FIGURÉ & Hipparion prostylum \\
\hline MNHN.F.LUB846 & crâne & LECTOTYPE & Hipparion prostylum \\
\hline MNHN.F.LUB847 & crâne & FIGURÉ & Hipparion prostylum \\
\hline MNHN.F.LUB848 & dentition supérieure & & Hipparion prostylum \\
\hline MNHN.F.LUB849 & crâne & & Hipparion prostylum \\
\hline MNHN.F.LUB850 & dentition supérieure & & Hipparion prostylum \\
\hline MNHN.F.LUB851 & dentition supérieure & & Hipparion prostylum \\
\hline MNHN.F.LUB852 & dentition supérieure & FIGURÉ & Hipparion prostylum \\
\hline MNHN.F.LUB853 & dentition supérieure & & Hipparion prostylum \\
\hline MNHN.F.LUB854 & dentition supérieure & & Hipparion prostylum \\
\hline MNHN.F.LUB855 & dentition supérieure & & Hipparion prostylum \\
\hline MNHN.F.LUB856 & dentition supérieure & & Hipparion prostylum \\
\hline MNHN.F.LUB857 & dentition supérieure & & Hipparion prostylum \\
\hline MNHN.F.LUB858 & dentition supérieure & & Hipparion prostylum \\
\hline MNHN.F.LUB859 & dentition supérieure & & Hipparion prostylum \\
\hline MNHN.F.LUB860 & dentition supérieure & & Hipparion prostylum \\
\hline MNHN.F.LUB861 & dentition supérieure & & Hipparion prostylum \\
\hline MNHN.F.LUB862 & dentition supérieure & & Hipparion prostylum \\
\hline MNHN.F.LUB863 & dentition supérieure & & Hipparion prostylum \\
\hline MNHN.F.LUB864 & dentition supérieure & & Hipparion prostylum \\
\hline MNHN.F.LUB865 & mandibule & FIGURÉ & Tragoportax amalthea \\
\hline MNHN.F.LUB866 & mandibule & & Hipparion prostylum \\
\hline MNHN.F.LUB867 & mandibule & & Hipparion prostylum \\
\hline MNHN.F.LUB868 & dentition inférieure & FIGURÉ & Hipparion prostylum \\
\hline MNHN.F.LUB869 & mandibule & FIGURÉ & Hipparion prostylum \\
\hline MNHN.F.LUB870 & mandibule & & Hipparion prostylum \\
\hline MNHN.F.LUB871 & mandibule & & Hipparion prostylum \\
\hline MNHN.F.LUB872 & dentition inférieure & & Hipparion prostylum \\
\hline MNHN.F.LUB873 & dentition inférieure & & Hipparion prostylum \\
\hline MNHN.F.LUB874 & dentition inférieure & & Hipparion prostylum \\
\hline MNHN.F.LUB875 & mandibule & & Hipparion prostylum \\
\hline MNHN.F.LUB876 & dentition inférieure & & Hipparion prostylum \\
\hline MNHN.F.LUB877 & vertèbre & & Hipparion prostylum \\
\hline MNHN.F.LUB878 & vertèbre & & Hipparion prostylum \\
\hline MNHN.F.LUB879 & vertèbre & & Hipparion prostylum \\
\hline MNHN.F.LUB880 & vertèbre & & Hipparion prostylum \\
\hline MNHN.F.LUB881 & vertèbre & & Hipparion prostylum \\
\hline MNHN.F.LUB882 & vertèbre & & Hipparion prostylum \\
\hline MNHN.F.LUB883 & ceinture & & Hipparion prostylum \\
\hline MNHN.F.LUB884 & ceinture & & Hipparion prostylum \\
\hline MNHN.F.LUB885 & stylopode & & Hipparion prostylum \\
\hline MNHN.F.LUB886 & stylopode & & Hipparion prostylum \\
\hline MNHN.F.LUB887 & stylopode & & Hipparion prostylum \\
\hline MNHN.F.LUB888a & zeugopode & & Hipparion prostylum \\
\hline MNHN.F.LUB888b & zeugopode & & Hipparion prostylum \\
\hline MNHN.F.LUB889a & zeugopode & & Hipparion prostylum \\
\hline MNHN.F.LUB889b & zeugopode & & Hipparion prostylum \\
\hline MNHN.F.LUB890a & zeugopode & & Hipparion prostylum \\
\hline MNHN.F.LUB890b & zeugopode & & Hipparion prostylum \\
\hline MNHN.F.LUB891 & basipode & & Hipparion prostylum \\
\hline MNHN.F.LUB892 & basipode & & Hipparion prostylum \\
\hline MNHN.F.LUB893 & basipode & & Hipparion prostylum \\
\hline
\end{tabular}


ANNEXE 1. - Suite.

\begin{tabular}{|c|c|c|c|}
\hline Numéro de spécimen & Anatomie & Statut nomenclatural & Espèce \\
\hline MNHN.F.LUB894 & basipode & & Hipparion prostylum \\
\hline MNHN.F.LUB895 & basipode & & Hipparion prostylum \\
\hline MNHN.F.LUB896 & basipode & & Hipparion prostylum \\
\hline MNHN.F.LUB897 & basipode & & Hipparion prostylum \\
\hline MNHN.F.LUB898a & basipode & & Hipparion prostylum \\
\hline MNHN.F.LUB898b & métapode & & Hipparion prostylum \\
\hline MNHN.F.LUB899 & métapode & & Hipparion prostylum \\
\hline MNHN.F.LUB900 & métapode & & Hipparion prostylum \\
\hline MNHN.F.LUB901 & métapode & & Hipparion prostylum \\
\hline MNHN.F.LUB902 & métapode & & Hipparion prostylum \\
\hline MNHN.F.LUB903 & métapode & & Hipparion prostylum \\
\hline MNHN.F.LUB904 & ceinture & & Hipparion prostylum \\
\hline MNHN.F.LUB905 & ceinture & & Hipparion prostylum \\
\hline MNHN.F.LUB906a & ceinture & & Hipparion prostylum \\
\hline MNHN.F.LUB906b & ceinture & & Hipparion prostylum \\
\hline MNHN.F.LUB907 & ceinture & & Hipparion prostylum \\
\hline MNHN.F.LUB908 & ceinture & & Hipparion prostylum \\
\hline MNHN.F.LUB909 & ceinture & & Hipparion prostylum \\
\hline MNHN.F.LUB910 & stylopode & & Hipparion prostylum \\
\hline MNHN.F.LUB911 & stylopode & & Hipparion prostylum \\
\hline MNHN.F.LUB912 & stylopode & & Hipparion prostylum \\
\hline MNHN.F.LUB913 & stylopode & & Hipparion prostylum \\
\hline MNHN.F.LUB914 & stylopode & & Hipparion prostylum \\
\hline MNHN.F.LUB915 & zeugopode & & Hipparion prostylum \\
\hline MNHN.F.LUB916 & zeugopode & & Hipparion prostylum \\
\hline MNHN.F.LUB917 & zeugopode & & Hipparion prostylum \\
\hline MNHN.F.LUB918a & zeugopode & FIGURÉ & Hipparion prostylum \\
\hline MNHN.F.LUB918b & basipode & FIGURÉ & Hipparion prostylum \\
\hline MNHN.F.LUB918c & basipode & FIGURÉ & Hipparion prostylum \\
\hline MNHN.F.LUB918d & basipode & FIGURÉ & Hipparion prostylum \\
\hline MNHN.F.LUB918e & basipode & FIGURÉ & Hipparion prostylum \\
\hline MNHN.F.LUB918f & basipode & FIGURÉ & Hipparion prostylum \\
\hline MNHN.F.LUB918g & métapode & FIGURÉ & Hipparion prostylum \\
\hline MNHN.F.LUB918h & métapode & FIGURÉ & Hipparion prostylum \\
\hline MNHN.F.LUB918i & métapode & FIGURÉ & Hipparion prostylum \\
\hline MNHN.F.LUB918j & phalange & FIGURÉ & Hipparion prostylum \\
\hline MNHN.F.LUB918k & phalange & FIGURÉ & Hipparion prostylum \\
\hline MNHN.F.LUB918I & phalange & FIGURÉ & Hipparion prostylum \\
\hline MNHN.F.LUB918m & phalange & FIGURÉ & Hipparion prostylum \\
\hline MNHN.F.LUB918n & phalange & FIGURÉ & Hipparion prostylum \\
\hline MNHN.F.LUB9180 & phalange & FIGURÉ & Hipparion prostylum \\
\hline MNHN.F.LUB918p & phalange & FIGURÉ & Hipparion prostylum \\
\hline MNHN.F.LUB918q & phalange & FIGURÉ & Hipparion prostylum \\
\hline MNHN.F.LUB918r & sésamoïde & FIGURÉ & Hipparion prostylum \\
\hline MNHN.F.LUB918s & sésamoïde & FIGURÉ & Hipparion prostylum \\
\hline MNHN.F.LUB919 & basipode & & Hipparion prostylum \\
\hline MNHN.F.LUB920 & basipode & & Hipparion prostylum \\
\hline MNHN.F.LUB921a & basipode & & Hipparion prostylum \\
\hline MNHN.F.LUB921b & basipode & & Hipparion prostylum \\
\hline MNHN.F.LUB922a & basipode & & Hipparion prostylum \\
\hline MNHN.F.LUB922b & basipode & & Hipparion prostylum \\
\hline MNHN.F.LUB922c & basipode & & Hipparion prostylum \\
\hline MNHN.F.LUB922d & basipode & & Hipparion prostylum \\
\hline MNHN.F.LUB922e & métapode & & Hipparion prostylum \\
\hline MNHN.F.LUB922f & métapode & & Hipparion prostylum \\
\hline MNHN.F.LUB923 & métapode & & Hipparion prostylum \\
\hline
\end{tabular}


ANNEXE 1. - Suite.

\begin{tabular}{|c|c|c|c|}
\hline Numéro de spécimen & Anatomie & Statut nomenclatural & Espèce \\
\hline MNHN.F.LUB924 & métapode & & Hipparion prostylum \\
\hline MNHN.F.LUB925a & métapode & & Hipparion prostylum \\
\hline MNHN.F.LUB925b & métapode & & Hipparion prostylum \\
\hline MNHN.F.LUB925c & métapode & & Hipparion prostylum \\
\hline MNHN.F.LUB925d & phalange & & Hipparion prostylum \\
\hline MNHN.F.LUB925e & phalange & & Hipparion prostylum \\
\hline MNHN.F.LUB925f & phalange & & Hipparion prostylum \\
\hline MNHN.F.LUB925g & phalange & & Hipparion prostylum \\
\hline MNHN.F.LUB925h & phalange & & Hipparion prostylum \\
\hline MNHN.F.LUB925i & phalange & & Hipparion prostylum \\
\hline MNHN.F.LUB925j & phalange & & Hipparion prostylum \\
\hline MNHN.F.LUB926 & phalange & FIGURÉ & Hipparion prostylum \\
\hline MNHN.F.LUB927 & phalange & & Hipparion prostylum \\
\hline MNHN.F.LUB928 & phalange & & Hipparion prostylum \\
\hline MNHN.F.LUB929 & phalange & & Hipparion prostylum \\
\hline MNHN.F.LUB930 & sésamoïde & & Hipparion prostylum \\
\hline MNHN.F.LUB931 & métapode & & Hipparion prostylum \\
\hline MNHN.F.LUB932 & métapode & & Hipparion prostylum \\
\hline MNHN.F.LUB933 & crâne & FIGURÉ & Dihoplus schleiermacher \\
\hline MNHN.F.LUB934 & crâne & & Dihoplus schleiermacher \\
\hline MNHN.F.LUB935 & dentition supérieure & & Dihoplus schleiermacher \\
\hline MNHN.F.LUB936 & mandibule & & Dihoplus schleiermacher \\
\hline MNHN.F.LUB937 & mandibule & FIGURÉ & Dihoplus schleiermacher \\
\hline MNHN.F.LUB938 & mandibule & & Dihoplus schleiermacher \\
\hline MNHN.F.LUB939 & mandibule & & Dihoplus schleiermacher \\
\hline MNHN.F.LUB940 & vertèbre & & Dihoplus schleiermacher \\
\hline MNHN.F.LUB941 & stylopode & & Dihoplus schleiermacher \\
\hline MNHN.F.LUB942 & zeugopode & & Dihoplus schleiermacher \\
\hline MNHN.F.LUB943a & zeugopode & FIGURÉ & Dihoplus schleiermacher \\
\hline MNHN.F.LUB943b & zeugopode & FIGURÉ & Dihoplus schleiermacher \\
\hline MNHN.F.LUB944 & basipode & & Dihoplus schleiermacher \\
\hline MNHN.F.LUB945 & basipode & FIGURÉ & Dihoplus schleiermacher \\
\hline MNHN.F.LUB946 & basipode & & Dihoplus schleiermacher \\
\hline MNHN.F.LUB947 & basipode & & Dihoplus schleiermacher \\
\hline MNHN.F.LUB948 & métapode & FIGURÉ & Dihoplus schleiermacher \\
\hline MNHN.F.LUB949a & métapode & FIGURÉ & Dihoplus schleiermacher \\
\hline MNHN.F.LUB949b & métapode & FIGURÉ & Dihoplus schleiermacher \\
\hline MNHN.F.LUB949c & métapode & FIGURÉ & Dihoplus schleiermacher \\
\hline MNHN.F.LUB949d & phalange & FIGURÉ & Dihoplus schleiermacher \\
\hline MNHN.F.LUB950 & métapode & & Dihoplus schleiermacher \\
\hline MNHN.F.LUB951 & ceinture & & Dihoplus schleiermacher \\
\hline MNHN.F.LUB952 & ceinture & FIGURÉ & Dihoplus schleiermacher \\
\hline MNHN.F.LUB953 & stylopode & FIGURÉ & Dihoplus schleiermacher \\
\hline MNHN.F.LUB954 & stylopode & & Dihoplus schleiermacher \\
\hline MNHN.F.LUB955a & zeugopode & FIGURÉ & Dihoplus schleiermacher \\
\hline MNHN.F.LUB955b & zeugopode & FIGURÉ & Dihoplus schleiermacher \\
\hline MNHN.F.LUB956a & basipode & FIGURÉ & Dihoplus schleiermacher \\
\hline MNHN.F.LUB956b & basipode & FIGURÉ & Dihoplus schleiermacher \\
\hline MNHN.F.LUB956c & basipode & FIGURÉ & Dihoplus schleiermacher \\
\hline MNHN.F.LUB956d & basipode & FIGURÉ & Dihoplus schleiermacher \\
\hline MNHN.F.LUB957 & métapode & & Dihoplus schleiermacher \\
\hline MNHN.F.LUB958a & métapode & FIGURÉ & Dihoplus schleiermacher \\
\hline MNHN.F.LUB958b & métapode & FIGURÉ & Dihoplus schleiermacher \\
\hline MNHN.F.LUB958c & métapode & FIGURÉ & Dihoplus schleiermacher \\
\hline MNHN.F.LUB959 & métapode & & Dihoplus schleiermacher \\
\hline MNHN.F.LUB960 & stylopode & FIGURÉ & Aceratherium incisivum \\
\hline
\end{tabular}


ANNEXE 1. - Suite.

\begin{tabular}{|c|c|c|c|}
\hline Numéro de spécimen & Anatomie & Statut nomenclatural & Espèce \\
\hline MNHN.F.LUB961 & zeugopode & & Aceratherium incisivum \\
\hline MNHN.F.LUB962 & zeugopode & FIGURÉ & Aceratherium incisivum \\
\hline MNHN.F.LUB963 & basipode & FIGURÉ & Aceratherium incisivum \\
\hline MNHN.F.LUB964a & basipode & FIGURÉ & Aceratherium incisivum \\
\hline MNHN.F.LUB964b & basipode & FIGURÉ & Aceratherium incisivum \\
\hline MNHN.F.LUB965 & basipode & & Aceratherium incisivum \\
\hline MNHN.F.LUB966a & métapode & FIGURÉ & Aceratherium incisivum \\
\hline MNHN.F.LUB966b & métapode & FIGURÉ & Aceratherium incisivum \\
\hline MNHN.F.LUB966c & métapode & FIGURÉ & Aceratherium incisivum \\
\hline MNHN.F.LUB967 & basipode & FIGURÉ & Deinotherium giganteum \\
\hline MNHN.F.LUB968 & basipode & & Deinotherium giganteum \\
\hline MNHN.F.LUB969 & basipode & & Deinotherium giganteum \\
\hline MNHN.F.LUB970 & métapode & FIGURÉ & Deinotherium giganteum \\
\hline MNHN.F.LUB971 & ceinture & & Incertae sedis \\
\hline MNHN.F.LUB972 & basipode & & Incertae sedis \\
\hline MNHN.F.LUB973 & ceinture & & Incertae sedis \\
\hline MNHN.F.LUB974 & os indet. & & Incertae sedis \\
\hline MNHN.F.LUB975 & stylopode & & Ictitherium sp. \\
\hline MNHN.F.LUB976 & sésamoïde & & Incertae sedis \\
\hline MNHN.F.LUB977 & stylopode & & Incertae sedis \\
\hline MNHN.F.LUB978 & stylopode & & Incertae sedis \\
\hline MNHN.F.LUB979 & stylopode & & Incertae sedis \\
\hline MNHN.F.LUB980 & ossicule dermique & & Cheirogaster leberonensis \\
\hline MNHN.F.LUB981 & ossicule dermique & & Cheirogaster leberonensis \\
\hline MNHN.F.LUB982 & os crural & & Cheirogaster leberonensis \\
\hline MNHN.F.LUB983 & dossiere & FIGURÉ & Testudo sp. \\
\hline MNHN.F.LUB984 & carapace & FIGURÉ & Testudo sp. \\
\hline MNHN.F.LUB985a & basipode & & Hipparion prostylum \\
\hline MNHN.F.LUB985b & basipode & & Hipparion prostylum \\
\hline MNHN.F.LUB985c & basipode & & Hipparion prostylum \\
\hline MNHN.F.LUB985d & basipode & & Hipparion prostylum \\
\hline MNHN.F.LUB985e & basipode & & Hipparion prostylum \\
\hline MNHN.F.LUB986a & métapode & & Hipparion prostylum \\
\hline MNHN.F.LUB986b & métapode & & Hipparion prostylum \\
\hline MNHN.F.LUB986c & métapode & & Hipparion prostylum \\
\hline MNHN.F.LUB987 & objet autre & FIGURÉ & bloc fossilifère \\
\hline MNHN.F.LUB987a & crâne & & Hipparion prostylum \\
\hline MNHN.F.LUB987b & mandibule & & Hipparion prostylum \\
\hline MNHN.F.LUB987c & phalange & FIGURÉ & Hipparion prostylum \\
\hline MNHN.F.LUB987d & zeugopode & FIGURÉ & Hipparion prostylum \\
\hline MNHN.F.LUB987e & stylopode & FIGURÉ & Hipparion prostylum \\
\hline MNHN.F.LUB987f1 & stylopode & FIGURÉ & Hipparion prostylum \\
\hline MNHN.F.LUB987f2 & zeugopode & FIGURÉ & Hipparion prostylum \\
\hline MNHN.F.LUB987f3 & basipode & FIGURÉ & Hipparion prostylum \\
\hline MNHN.F.LUB987f4 & basipode & FIGURÉ & Hipparion prostylum \\
\hline MNHN.F.LUB987f5 & basipode & FIGURÉ & Hipparion prostylum \\
\hline MNHN.F.LUB987f6 & basipode & FIGURÉ & Hipparion prostylum \\
\hline MNHN.F.LUB987f7 & métapode & FIGURÉ & Hipparion prostylum \\
\hline MNHN.F.LUB987f8 & métapode & FIGURÉ & Hipparion prostylum \\
\hline MNHN.F.LUB987g & basipode & FIGURÉ & Hipparion prostylum \\
\hline MNHN.F.LUB987h & zeugopode & FIGURÉ & Hipparion prostylum \\
\hline MNHN.F.LUB987i1 & basipode & FIGURÉ & Hipparion prostylum \\
\hline MNHN.F.LUB987i2 & métapode & FIGURÉ & Hipparion prostylum \\
\hline MNHN.F.LUB987i3 & métapode & FIGURÉ & Hipparion prostylum \\
\hline MNHN.F.LUB987j & métapode & FIGURÉ & Hipparion prostylum \\
\hline MNHN.F.LUB987k1 & métapode & FIGURÉ & Hipparion prostylum \\
\hline
\end{tabular}


ANNEXE 1. - Suite et fin.

\begin{tabular}{llll}
\hline Numéro de spécimen & Anatomie & Statut nomenclatural & Espèce \\
\hline MNHN.F.LUB987k2 & métapode & FIGURÉ & Hipparion prostylum \\
MNHN.F.LUB987I1 & zeugopode & FIGURÉ & Hipparion prostylum \\
MNHN.F.LUB987I2 & basipode & FIGURÉ & Hipparion prostylum \\
MNHN.F.LUB987I3 & basipode & FIGURÉ & Hipparion prostylum \\
MNHN.F.LUB987m & ceinture & FIGURÉ & Hipparion prostylum \\
MNHN.F.LUB987n1 & métapode & FIGURÉ & Hipparion prostylum \\
MNHN.F.LUB987n2 & phalange & FIGURÉ & Hipparion prostylum \\
MNHN.F.LUB987n3 & phalange & FIGURÉ & Hipparion prostylum \\
MNHN.F.LUB987n4 & phalange & FIGURÉ & Hipparion prostylum \\
MNHN.F.LUB987n5 & métapode & FIGURÉ & Hipparion prostylum \\
MNHN.F.LUB987n6 & phalange & FIGURÉ & Hipparion prostylum \\
MNHN.F.LUB987n7 & phalange & FIGURÉ & Hipparion prostylum \\
MNHN.F.LUB987n8 & phalange & FIGURÉ & Hipparion prostylum \\
MNHN.F.LUB987n9 & basipode & FIGURÉ & Hipparion prostylum \\
MNHN.F.LUB98701 & métapode & FIGURÉ & Hipparion prostylum \\
MNHN.F.LUB98702 & métapode & FIGURÉ & Hipparion prostylum \\
MNHN.F.LUB987p & stylopode & FIGURÉ & Hipparion prostylum \\
MNHN.F.LUB987q & basipode & FIGURÉ & Aceratherium incisivum \\
MNHN.F.LUB987r & crâne & FIGURÉ & Gazella deperdita \\
MNHN.F.LUB987s1 & dentition supérieure & FIGURÉ & Gazella deperdita \\
MNHN.F.LUB987s2 & dentition indéterminée & FIGURÉ & Gazella deperdita \\
MNHN.F.LUB987t & mandibule & FIGURÉ & Palaeoreas lindermayeri \\
MNHN.F.LUB987u1 & os indet. & FIGURÉ & Incertae sedis \\
MNHN.F.LUB987u2 & os indet. & FIGURÉ & Incertae sedis \\
\hline
\end{tabular}

ANNEXE 2. - Spécimens manquants et numéros invalides dans et hors catalogue.

NumÉROS INVALIDES DANS LE CATALOGUE

LUB 130 : spécimen provenant de Pikermi (Grèce);

LUB 134 : spécimen provenant de Pikermi (Grèce);

LUB 232 : spécimen provenant de Pikermi (Grèce);

LUB 245 : spécimen de provenance inconnue.

\section{SPÉCIMENS MANQUANTS DANS LE CATALOGUE}

LUB 54 : intervalle de numérotation des " Hipparions ";

LUB 138 : intervalle de numérotation des " Hipparions »;

LUB 182 : Hipparion - incisive (d'après un inventaire papier) ;

LUB 187 : Hipparion - P3 ou P4 supérieure gauche (d'après un inventaire papier) ;

LUB 193 : Hipparion - P2 supérieure droite (d'après un inventaire papier) ;

LUB 366 : Hipparion - magnum (d'après un inventaire papier).

SpéCIMENS MANQUANTS NON CATAlOGUÉS, TIRÉS DE GAUdRY (1873) ET HeinTZ (1971)

Tragoportax amalthea - crâne \& chevilles - Figuré dans Gaudry (1873: pl. 10, fig. 3) ;

Tragoportax amalthea - basicrâne - Figuré dans Gaudry (1873: pl. 10, fig. 4) ;

Testudo sp. - carapace - Figuré dans Gaudry (1873: pl. 14, fig. 3) ;

Gazella deperdita - p3 inférieure gauche - Figuré dans Heintz (1971: pl. 4, fig. 8) ;

Gazella deperdita - p4 inférieure gauche - Figuré dans Heintz (1971: pl. 4, fig. 9). 
ANNEXE 3. - Listes fauniques des artiodactyles, carnivores, périssodactyles, proboscidiens et chéloniens du Mont Luberon. Les binoms utilisés par Gaudry (1873) sont entre parenthèses ; les points d'interrogation sont de Gaudry.

\section{ARTIODACTYLES}

Pliocervus matheroni (Gervais, 1859) (= Cervus matheronis) ;

Gazella deperdita (Gervais, 1847);

Helladotherium duvernoyi (Gaudry \& Lartet, 1856) ;

Microstonyx major (Gervais, 1848) (= Sus major) ;

Tragoportax amalthea (Roth \& Wagner, 1854) (= Tragocerus amaltheus) ;

? Palaeoreas lindermayeri (Wagner, 1848).

\section{CARNIVORES}

Adcrocuta eximia (Roth \& Wagner, 1854) (= Hyaena eximia);

Hyaenictitherium wongii (Zdansky, 1924) (= Ictitherium hipparionum);

Amphimachairodus giganteus (Wagner, 1848) (= Machairodus cultridens);

? Plioviverrops orbignyi (Gaudry, 1862) (= Ictitherium orbignyi ?).

\section{PÉRISSODACTYLES}

? Aceratherium incisivum Kaup, 1832 (= Acerotherium incisivum ?) ;

Dihoplus schleiermacheri (Kaup, 1832) (= Rhinoceros schleiermacheri) ;

Hipparion prostylum Gervais, 1849 (= Hipparion gracile).

PROBOSCIDIENS

Deinotherium giganteum Kaup, 1829 (= Dinotherium giganteum).

CHÉLONIENS

Cheirogaster leberonensis (Depéret, 1890);

Testudo sensu lato. 